\title{
Frontier Research in Astrophysics (A Review from a personal point of view)
}

\author{
Franco Giovannelli*† \\ INAF - Istituto di Astrofisica e Planetologia Spaziali, Via del Fosso del Cavaliere, 100, 00133 \\ Roma, Italy \\ E-mail: Eranco.giovannellieiaps.inaf.it
}

\section{Lola Sabau-Graziati}

INTA-Dpt. Cargas Utiles y Ciencias del Espacio, C/ra de Ajalvir, Km 4 - E28850 Torrejón de Ardoz, Madrid, Spain

E-mail: sabaumdeinta.es

This talk is a summary of an updated version of the book The Impact of the Space Experiments on Our Knowledge of the Physics of the Universe, published in 2004 by the Kluwer Academic Publishers, reprinted from the review paper by Giovannelli, F. \& Sabau-Graziati, L.: 2004, Space Sci. Rev. 112, 1-443 (GSG2004), and subsequent considered lucubrations.

The structure of this review paper is the following:
1. Antecedent fact
2. Introduction
3. How to know our Universe
4. The use of wisdom in Physics
5. The present situation about the knowledge of the physics of our Universe
6. Habitable zone in the Milky Way and exoplanets
7. Origin of terrestrial life
8. Conclusions

Frontier Research in Astrophysics - III (FRAPWS2018)

28 May - 2 June 2018

Mondello (Palermo), Italy

* Speaker.

${ }^{\dagger}$ A footnote may follow. 


\section{Antecedent fact}

If we have not experimental information about the cross sections of nuclear reactions occurring in the stars it is hard and hazardous to describe the correct star evolution.

\subsection{Nuclear Reactions in Stars}

The knowledge of the cross-sections of nuclear reactions occurring in the stars appears as one of the most crucial points of all astroparticle physics. Direct measurements of the cross sections of the ${ }^{3} \mathrm{He}\left({ }^{4} \mathrm{He}, \gamma\right){ }^{7} \mathrm{Be}$ and ${ }^{7} \mathrm{Be}(\mathrm{p}, \gamma){ }^{8} \mathrm{Be}$ reactions of the $p p$ chain and ${ }^{14} \mathrm{~N}(\mathrm{p}, \gamma){ }^{15} \mathrm{O}$ reaction of the CNO-cycle will allow a substantial improvement in our knowledge on stellar evolution.

Wolschin (2003) published a very interesting paper about the history of the "Thermonuclear Processes in Stars and Stellar Neutrinos".

An impressive review about nuclear reactions (the pp chain and CNO cycles) has been published by Adelberger et al. (2011). They summarize and critically evaluate the available data on nuclear fusion cross sections important to energy generation in the Sun and other hydrogen-burning stars and to solar neutrino production. Recommended values and uncertainties are provided for key cross sections, and a recommended spectrum is given for ${ }^{8} \mathrm{~B}$ solar neutrinos. They also discuss opportunities for further increasing the precision of key rates, including new facilities, new experimental techniques, and improvements in theory. This review, which summarizes the conclusions of a workshop held at the Institute for Nuclear Theory, Seattle, in January 2009, is intended as a 10-year update and supplement to the reviews by Adelberger et al. (1998).

At the moment the LUNA (Laboratory for Underground Nuclear Astrophysics) is devoted to measure nuclear cross sections relevant in astroparticle physics. It is the most valuable experiment running underground in the Gran Sasso Laboratory of the INFN. Reviews about LUNA experiment have been published by Broggini et al. (2010; 2018).

The LUNA collaboration has already measured with good accuracy the key reactions $\mathrm{D}(\mathrm{p}, \gamma)^{3} \mathrm{He}$, ${ }^{3} \mathrm{He}(\mathrm{D}, \mathrm{p}){ }^{4} \mathrm{He}$ and ${ }^{3} \mathrm{He}\left({ }^{4} \mathrm{He}, \gamma\right){ }^{7} \mathrm{Be}$. These measurements substantially reduce the theoretical uncertainty of $\mathrm{D},{ }^{3} \mathrm{He},{ }^{7} \mathrm{Li}$ abundances. The $\mathrm{D}\left({ }^{4} \mathrm{He}, \gamma\right){ }^{6} \mathrm{Li}$ cross section - which is the key reaction for the determination of the primordial abundance of ${ }^{6} \mathrm{Li}$ - has been measured (e.g. Gustavino, 2007, 2009, 2011, 2012, 2013), as well as that of ${ }^{2} \mathrm{H}(\alpha, \gamma){ }^{6} \mathrm{Li}$ (Anders et al., 2013), and ${ }^{2} \mathrm{H}(\alpha, \gamma){ }^{6} \mathrm{Li}$ (Anders et al., 2014).

Other reactions fundamental for a better knowledge of stellar evolution have been studied by the LUNA experiment: e.g. ${ }^{17} \mathrm{O}(\mathrm{p}, \gamma){ }^{18} \mathrm{~F}$ (Scott et al. 2012); ${ }^{25} \mathrm{Mg}(\mathrm{p}, \gamma){ }^{26} \mathrm{Al}$ (Strieder et al., 2012) ${ }^{25} \mathrm{Mg}(\mathrm{p}, \gamma){ }^{26} \mathrm{Al}$ (Straniero et al., 2013); ${ }^{17} \mathrm{O}(\mathrm{p}, \gamma){ }^{18} \mathrm{~F}$ (Di Leva et al., 2014).

Cavanna et al. (2015) studied with the LUNA experiment the ${ }^{22} \mathrm{Ne}(\mathrm{p}, \gamma)^{23} \mathrm{Na}$ reaction that takes part in the neon-sodium cycle of hydrogen burning. This cycle affects the synthesis of the elements between ${ }^{20} \mathrm{Ne}$ and ${ }^{27} \mathrm{Al}$ in asymptotic giant branch stars and novae. They found a new reaction rate a factor of 5 higher than the recent evaluation at temperatures relevant to novae and asymptotic giant branch stars nucleosynthesis.

Depalo et al. (2016) performed direct measurements of the ${ }^{22} \mathrm{Ne}(\mathrm{p}, \gamma)^{23} \mathrm{Na}$ resonances with the LUNA experiment. Based on the present experimental data and also previous literature data, an updated thermonuclear reaction rate is provided in tabular and parametric form. The new-reaction rate is significantly higher than previous evaluations at temperatures of $0.08-0.3 \mathrm{GK}$. 
The ${ }^{17} \mathrm{O}(\mathrm{p}, \alpha){ }^{14} \mathrm{Na}$ reaction plays a key role in various astrophysical scenarios, from asymptotic giant branch stars to classical novae. It affects the synthesis of rare isotopes such as ${ }^{17} \mathrm{O}$ and ${ }^{18} \mathrm{~F}$, which can provide constraints on astrophysical models. Bruno et al. (2016) performed direct determination of the resonance strength $\mathrm{E}_{\mathrm{R}}=64.5 \mathrm{keV}$ of that reaction at the LUNA accelerator. They found a factor of 2 increase in the reaction rate at astrophysical temperatures relevant to shell hydrogen burning in red giant and asymptotic giant branch stars. The new rate implies lower ${ }^{17} \mathrm{O} /{ }^{16} \mathrm{O}$ ratios, with important implications on the interpretation of astrophysical observable quantities from these stars, as deeply discussed by Straniero et al. (2017).

In order to further remark the importance of LUNA measurement, we would like to mention the crucial problem of the correct prediction of the abundances of the light nuclides produced during the epoch of Big Bang Nucleosynthesis (BBN) which is one of the main topics of modern cosmology. Trezzi et al. (2017) report results about the cross section of the ${ }^{2} \mathrm{H}(\alpha, \gamma)^{6} \mathrm{Li}$ reaction that controls ${ }^{6} \mathrm{Li}$ production in the Big Bang. The cross section has been directly measured at the energies of interest for $\mathrm{BBN}$ for the first time, at center-of-mass energy $\mathrm{E}_{\mathrm{cm}}=80,93,120$, and 133 $\mathrm{keV}$. They found that the ${ }^{2} \mathrm{H}(\alpha, \gamma)^{6} \mathrm{Li}$ thermonuclear reaction rate is even lower than previously reported, thus increasing the discrepancy between predicted Big Bang ${ }^{6} \mathrm{Li}$ abundance and the amount of primordial ${ }^{6} \mathrm{Li}$ inferred from observations.

A general data base for Experimental Nuclear Reaction Data (EXFOR) can be found in: https://www-nds.iaea.org/exfor/exfor.htm.

\section{Introduction}

The Bridge between the Big Bang and Biology undoubtedly exists, as discussed in the book edited by Giovannelli (2001a). The big problem is how to cross this bridge, and the main question is: what are the experimental tools for understanding the pillars of this Bridge?

In order to cross this bridge, as always when we cross a bridge, we MUST advance slowly, step by step, with continuity, because everything is smoothly linked in the "magma" of the Universe, from the infinitely small to infinitely big, as sketched in Fig. 1 (Rees, 1988) "Origins. The Darwin College lectures (1986)" (Fabian, 1988).

Indeed, if we look at the Fig. 2 (left panel) - where a section of the metabolic network of a "simple" bacterium is shown - we can note that each point (each chemical compound) is connected to any other point through the complexity of the network (Luisi \& Capra, 2014) exactly the same occurring in the "cosmic network" where each point is connected to any other point through the complexity of the network as shown in Fig. 2 (right panel) (https://it.wikipedia.org/wiki/Cosmologia del plasma). The large-scale structure of the Universe, as traced by the distribution of galaxies, is now being revealed by large-volume cosmological surveys. The structure is characterized by galaxies distributed along filaments, the filaments connecting in turn to form a percolating network. Shandarin, Habib \& Heitmann (2010) objective was to quantitatively specify the underlying mechanisms that drive the formation of the cosmic network. By combining percolation-based analyses with N-body simulations of gravitational structure formation, they elucidate how the network has its origin in the properties of the initial density field (nature) and how its contrast is then amplified by the nonlinear mapping induced by the gravitational instability (nurture). 


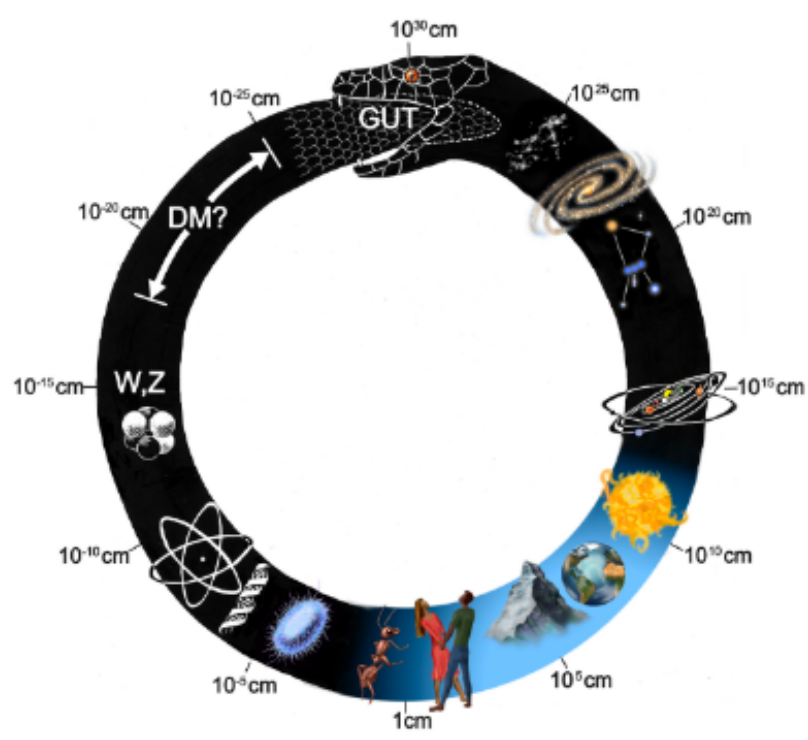

Figure 1: From the infinitely small to infinitely big (adopted by Rees, 1988).
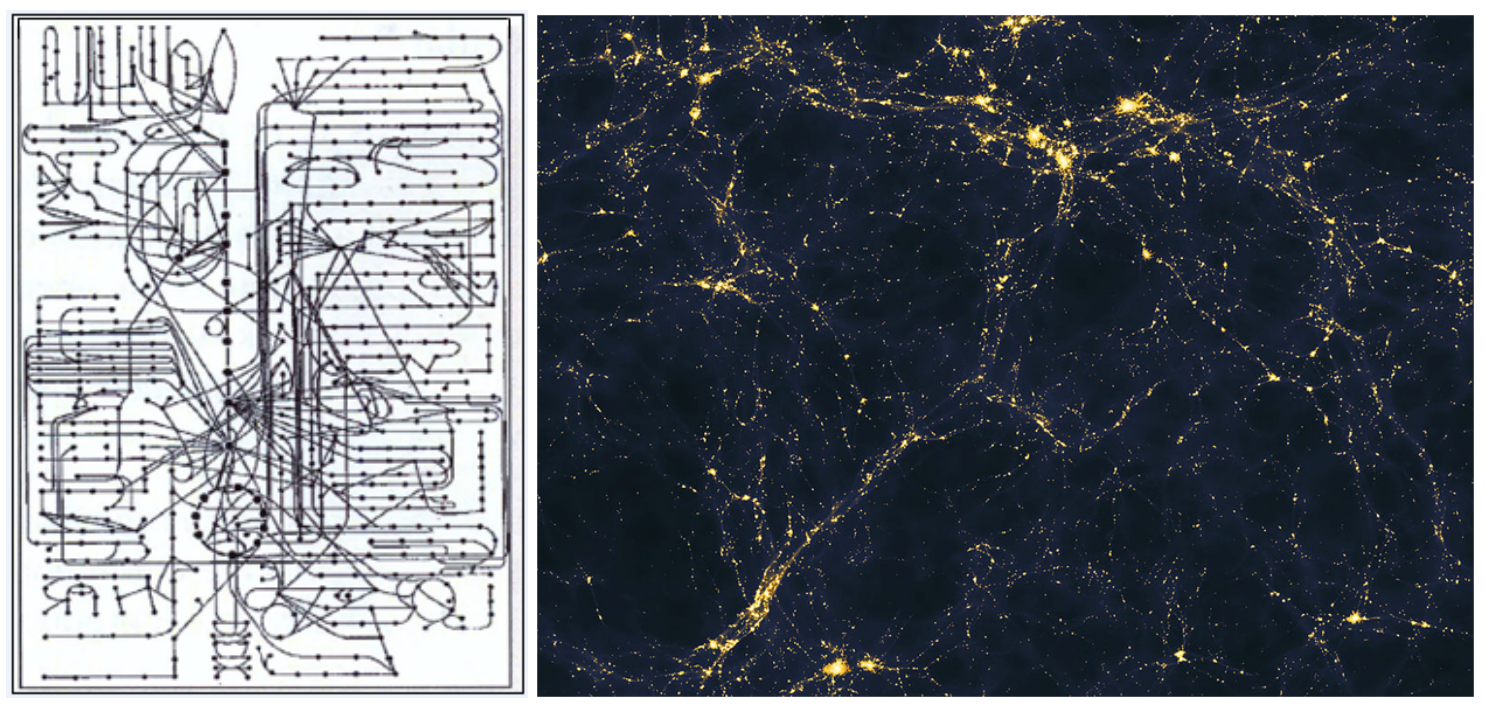

Figure 2: Left panel: Section of the metabolic network of a "simple" bacterium (Luisi \& Capra, 2014). Right panel: the "cosmic network" (https://it.wikipedia.org/wiki/Cosmologia del plasma).

All the components of the Universe follow the same cycle: birth, growth, aging, death as the living beings. Therefore for a complete understanding of the history of the Universe it is necessary to search along that cycle. For this purpose it is mandatory the use of wisdom in Physics, and not only in Physics.

As Albert Einstein affirmed, we can't solve problems by using the same kind of thinking we used when we created them. We can add something more, by using the wisdom: we can attach each kind of problem in a way as general as possible, and in any case it is necessary to go on without blinkers. 


\subsection{Accretion Processes in Cosmic Sources}

Accretion is a universal phenomenon that takes place in the vast majority of astrophysical objects. The progress of ground-based and space-borne observational facilities has resulted in the great amount of information on various accreting astrophysical objects, collected within the last decades. The accretion is accompanied by the process of extensive energy release that takes place on the surface of an accreting object and in various gaseous envelopes, accretion disk, jets and other elements of the flow pattern. The results of observations inspired the intensive development of accretion theory, which, in turn, enabled us to study unique properties of accreting objects and physical conditions in the surrounding environment. One of the most interesting outcomes of this intensive study is the fact that accretion processes are, in a sense, self-similar on various spatial scales from planetary systems to galaxies.

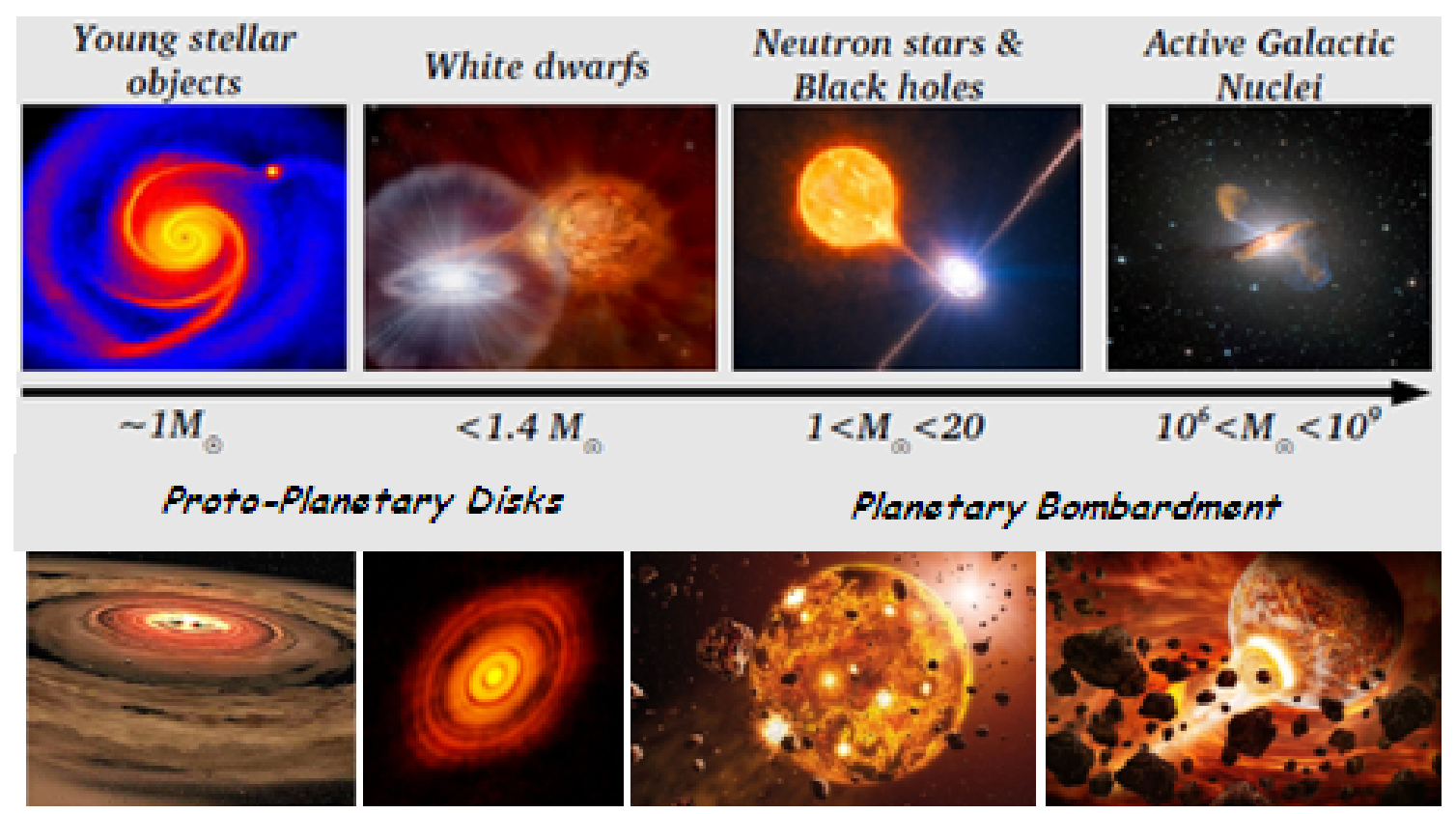

Figure 3: Accretion processes in different cosmic sources (adopted from Giovannelli \& Sabau-Graziati, 2016a, after Scaringi, 2015).

This fact gives us new opportunities to investigate objects that, by various reasons, are not available for direct study.

Cataclysmic variable stars are unique natural laboratories where one can conduct the detailed observational study of accretion processes and accretion disks.

Figure 3 shows a sketch of cosmic systems where accretion processes occur (Giovannelli \& Sabau-Graziati, 2016a, after Scaringi, 2015).

An international workshop on Accretion Processes in Cosmic Sources: Young Stellar Objects, Cataclysmic Variables (CVs) and Related Objects, X-ray Binary Systems, Active Galactic Nuclei was organized by us in collaboration with several colleagues of different international institutions, and took place in Saint Petersburg (Russian Federation) on September 2016. The proceedings discuss in details the physics of accretion processes in all the cosmic sources shown in Fig. 3 
(Giovannelli \& Sabau-Graziati, 2016b). The second edition of this workshop took place in St. Petersburg in the first week of September 2018.

Among the cosmic systems where accretion processes occur, undoubtedly, non-magnetic CVs, intermediate polars and polars constitute the most powerful probe to test our theories of the various modes of accretion. The reason is rather simple: $\mathrm{CVs}$ are enough close to us and their processes develop in time-scales relatively easy to be followed and enough energetic to be easily detected. The long term evolution of CV systems accreting at a prohibitive rate has become a hot topic both in terms of the fate of such systems (all sorts of supernovae) and the microphysics of Eddington and super Eddington mass accretion and mass loss flows. In particular we stress one of the hottest topics in present day astrophysics, namely the progenitors of SN-Ia. This problem is connected with fundamental issues in cosmology. Novae and recurrent novae are the most promising progenitor candidates but so far could not be nailed down. One of the most important goals of our serial workshops about The Golden Age of Cataclysmic Variables and Related Objects is to discuss what is missing in our knowledge of CVs that will allow a unique determination and observational confirmation (Giovannelli \& Sabau-Graziati, 2012, 2015a,b, 2018a). Indeed the sub-class of CVs, named Classical Novae $(\mathrm{CNe})$, which are the third most powerful stellar explosions in a galaxy, have been observed as close as a kpc and as far as galaxies in the Fornax cluster.

The recent renaissance in $\mathrm{CNe}$ studies is coming from the observations with 8-10m class telescopes, high resolution spectroscopy, in synergy with observations from space carried out with Swift, XMM, Chandra, HST, and Spitzer. This, coupled with recent advances in the theory of the outburst, can provide important advancement in the knowledge of the physics governing such a powerful accretors.

Since long time it appeared evident that the old classification of CVs based on the optical outburst properties, by which one may distinguish four groups:

- classical novae;

- recurrent novae;

- dwarf novae;

- nova-like objects

is neither self-consistent nor adequate and it is much better to consider primarily the observed accretion behaviour (Smak, 1985a,b). Therefore, it is necessary to find a method as general as possible to describe the behavior of cataclysmic variables. This can be obtained looking at the "Accretion Behaviour" and "Magnetic Fields".

\subsection{The Scenario Machine}

If we observe the nature in all its manifestations we discover that it always shows continuity. Then we have to abandon the "convenient method" of thinking everything in Watertight Compartments and to go toward a general model for compact accreting stars.

This was the fundamental concept of The Scenario Machine, an idea developed by Vladimir Lipunov and collaborators many years ago. 


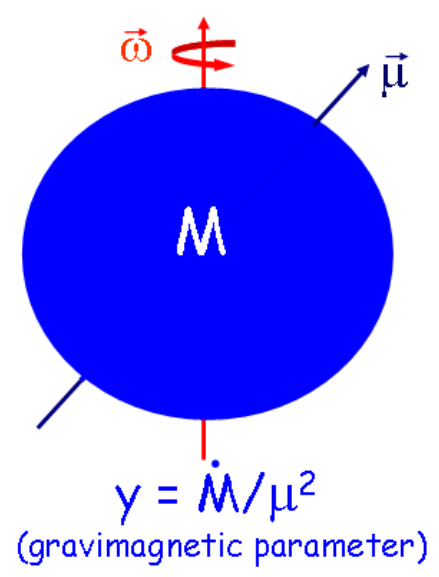

Figure 4: Gravimagnetic rotator: a body with mass $M$, having a magnetic moment $\vec{\mu}$, rotating with rotational velocity $\vec{\omega}$ (Giovannelli, 2016). The parameter $y=\dot{M} / \mu^{2}$ is called gravimagnetic parameter (Lipunov, 1987; Lipunov \& Postnov, 1988).

Starting from the trivial definition of X-ray Binary Systems (XRBs): they are binary systems emitting X-rays, a natural question arises. Are these systems governed by few physical parameters independent of their nature? The answer is positive. Indeed, High Mass XRBs (HMXRBs), Low Mass XRBs (LMXRBs), Anomalous X-ray Pulsars (AXPs), and Cataclysmic Variables (CVs) can be considered as gravimagnetic rotators: a body with mass $\mathrm{M}$, having a magnetic moment $\vec{\mu}$, rotating with rotational velocity $\vec{\omega}$, being the two axis not necessarily coincident, as sketched in Fig. 4 (Giovannelli, 2016). Introducing a physical parameter, $y=\dot{M} / \mu^{2}$, named gravimagnetic parameter, all the gravimagnetic rotators are contained in a plane $\log \mathrm{P}_{\text {spin }}$ vs $\log \mathrm{y}$ (Lipunov, 1987; Lipunov \& Postnov, 1988).

The Scenario Machine (Monte Carlo simulations of binary evolution) permits to build up the complete picture of all possible evolutionary stages of binaries in the Galaxy. The basic evolution equation (2.1) used for 500,000 systems containing magnetized stars provided the results contained in the plane $\log \mathrm{P}_{\text {spin }}-\log \mathrm{y}$, reported in the upper panel of Fig. 5. $\mathrm{P}_{\text {spin }}$ is expressed in seconds and the gravimagnetic parameter is expressed in unit of $10^{-42} \mathrm{~g} \mathrm{~s}^{-1} \mathrm{G}^{-2} \mathrm{~cm}^{-6}$. The symbols used for the different types of binaries are explained in the lower panel of Fig. 5. The definition of the characteristic radii can be found in the paper by Lipunov (1987). Observational examples of various types of rotators are reported in Fig. 6 (Lipunov, 1987).

$$
\frac{\mathrm{dI} \omega}{\mathrm{dt}}=\dot{\mathrm{M}} \mathrm{K}_{\mathrm{su}}-\frac{\kappa_{\mathrm{t}} \mu^{2}}{\mathrm{R}_{\mathrm{t}}^{3}}
$$

where:

$\mathrm{K}_{\mathrm{su}}=$ specific angular momentum applied by the accretion matter to the rotator;

$\mathrm{K}_{\mathrm{su}}=\sqrt{\mathrm{GM}_{\mathrm{x}} \mathrm{R}_{\mathrm{d}}}$ for Keplerian disk accretion;

$\mathrm{K}_{\mathrm{su}}=\eta_{\mathrm{t}} \Omega R_{\mathrm{g}}^{2}$ for wind accretion in a binary;

$\mathrm{K}_{\mathrm{su}} \sim 0$ for a single magnetic rotator;

$\mathrm{R}_{\mathrm{d}}=$ radius of the inner disk edge; 


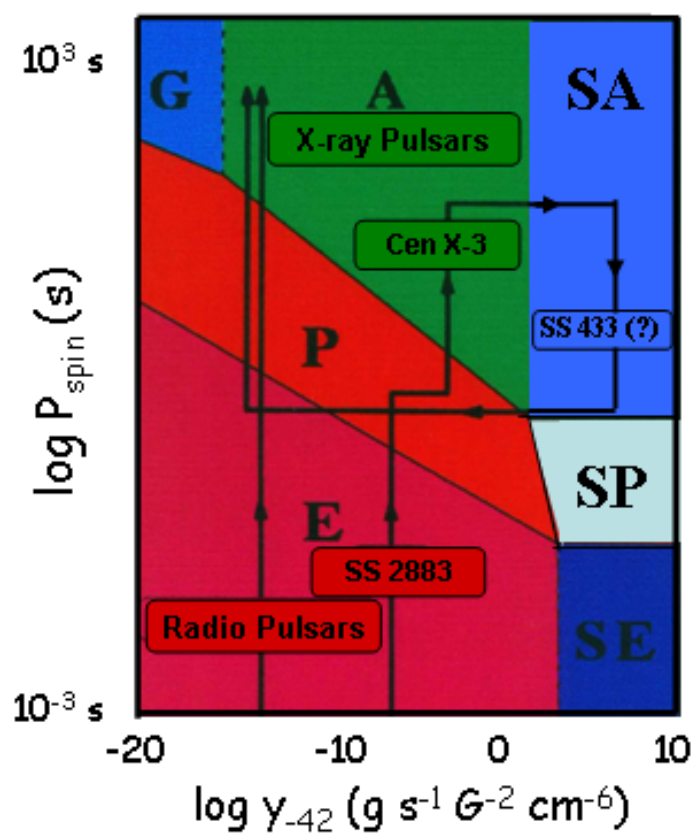

The rotator classification

\begin{tabular}{|c|c|c|}
\hline Designation & Name & Physical sense \\
\hline $\mathrm{E}$ & Ejector & $R_{s t}>\max \left\{R_{G}, R_{l}\right\}$ \\
\hline $\mathbf{P}$ & Propeller & $R_{c}<R_{s t} \leq \max \left\{R_{G}, R_{l}\right\}$ \\
\hline A & Accretor & $R_{s t} \leq R_{G}$ and $R_{s t} \leq R_{c} \quad \dot{M}_{c} \leq \dot{M}_{c r}$ \\
\hline G & Georotator & $R_{G}<R_{s t} \leq R_{c}$ \\
\hline M & Magnetor & $R_{s t}>a$ and $R_{c}>a$ \\
\hline SE & Superejector & $R_{s t}>R_{l}$ \\
\hline SP & Superpropeller & $R_{c}<R_{s t} \leq R_{l}$ \\
\hline SA & Superaccretor & $R_{s t} \leq R_{c}$ and $R_{s t} \leq R_{G}$ \\
\hline
\end{tabular}

Figure 5: Upper panel: distribution of magnetic rotators in the plane "Spin Period" - "Gavimagnetic Parameter" (adapted from Lipunov, 1995); lower panel: classification of rotators (Lipunov, 1987).

\begin{tabular}{|c|c|c|c|}
\hline Type of rotator & Designation & $\begin{array}{l}\text { The clearly confirmed } \\
\text { observational example }\end{array}$ & Model assumptions \\
\hline Ejector & $\mathrm{E}$ & Radiopulsars & $\begin{array}{l}\text { LSI }+61^{\circ} 303, \text { Cyg X-3, } \\
\text { BL Lac objects, }\end{array}$ \\
\hline Propeller & $P$ & - & $\begin{array}{l}\text { Transient X-ray sources, } \\
\gamma \text {-bursts, some cataclysmic } \\
\text { variables (dwarf novae), } \\
\text { magnetic Ap-stars }\end{array}$ \\
\hline Accretor & A & $\begin{array}{l}\text { X-ray pulsars, X-ray } \\
\text { bursters, cataclysmic } \\
\text { variables with white } \\
\text { dwarfs, novae, intermediate } \\
\text { polars }\end{array}$ & - \\
\hline Superejector & SE & - & SS 433, AGN, QSO \\
\hline Superpropeller & SP & - & - \\
\hline Superaccretor & SA & - & SS 433 \\
\hline Georotator & $\mathrm{G}$ & - & - \\
\hline Magnetor & M & Polars & - \\
\hline
\end{tabular}

Figure 6: Observational examples of rotators (Lipunov, 1987). 
$\Omega=$ rotational frequency of the binary system;

$\eta_{\mathrm{t}}=1 / 4 \quad$ (Illarionov \& Sunyaev, 1975);

$\kappa_{\mathrm{t}}=$ dimensionless factor;

$\mathrm{R}_{\mathrm{t}}=$ characteristic radius;

$\dot{M}=$ accretion rate in different regimes.

Using the "Scenario Machine" Raguzova \& Lipunov (1999) obtained an evolutionary track that can lead to the formation of $\mathrm{Be} / \mathrm{BH}$ systems. The modern evolutionary scenario predicts the existence of binary black holes on eccentric orbits around Be stars and such systems may be discovered in the near future... Like happened! Indeed MWC 656, the first Be/BH binary was detected by Casares et al. (2014).

Raguzova \& Lipunov (1999) calculations show that binary black holes with Be stars must have $0.2<\mathrm{e}<0.8$. It is particularly difficult to detect such systems as most of their spectroscopic variations occur in a relatively small portion of the orbit, and could easily be missed if the systems are observed at widely separated epochs. They found for 20-30 Be/NS systems the formation of 1 Be/BH system.

Belczynski \& Ziółkowski (2009) used binary population synthesis models to show that the expected ratio of $\mathrm{Be} / \mathrm{XRBs}$ with neutron stars to black holes in the Galaxy is relatively high: for $~$ $30-50 \mathrm{Be} / \mathrm{NS}$ systems the formation of $1 \mathrm{Be} / \mathrm{BH}$ system, and so broadly in line with observations.

\subsection{Composition of the Universe}

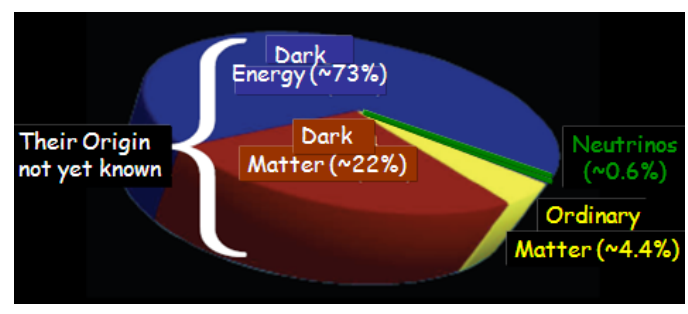

Figure 7: The cosmic budget.

A fundamental question naturally arises: what is now the situation about our knowledge of the Universe? The answer is discouraging: we know a very small part of it, and not very well.

Indeed, Fig. 7 shows schematically such a situation. We can really discuss only on $\sim 5 \%$ of the content of the Universe. The remnant of $\sim 95 \%$ is almost completely unknown. However, the recent fundamental progress in gravitational astronomy could open an incredible source of information about this "unknown" content.

\section{How to know our Universe}

The Universe manifests not only through electromagnetic radiation but also through astroparticles, including neutrinos, and recently through gravitational waves. Therefore, multifrequency observations, possibly simultaneous, are fundamental in photonic astrophysics, particle astrophysics, and gravitational wave astrophysics. All information coming from multifrequency observations are fundamental for understanding the main pillars of the Bridge between the Big Bang and Biology. 
The big problem is how to cross this bridge, and the main question is: what are the experimental tools for understanding the pillars of this Bridge?

In the following we will briefly discuss the main pillars of this bridge by using the huge amount of experimental data coming from "Active Physics Experiments (APEs)" and from "Passive Physics Experiments (PPEs)", both ground- and space-based. The APEs try to reproduce in the laboratory the physical conditions of our Universe at the beginning of its life and later, while the PPEs try to observe our Universe after the epoch of recombination, when the Cosmic Microwave Background (CMB) gives witness of the conditions of the primeval Universe, and later - after the epoch of reionization, when the first stars appear - for providing information about the formation of galaxies, "active" and "normal", quasars (QSOs), and all the processes giving rise to Gamma Ray Bursts (GRBs), stellar evolution and Supernovae explosions. These latter phenomena are responsible of the injection of heavy elements in the interstellar medium, the condition necessary for the formation of rocky planets, and thus for the flowering of the life.

Thus it is evident the necessity of many kinds of experiments in different frequency regions. Upstream of this we have to distinguish two great classes of experiments: big experiments and small experiments.

\subsection{Big experiments}

Several examples of big experiments are:

- GAIA (distances of billions stars);

- European-Extreme Large Telescope: E-ELT (the next generation ground telescope);

- James Webb Space Telescope: JWST (the next generation space telescope);

- Canadian Hydrogen Intensity Mapping Experiment (CHIME) (Fast Radio Bursts);

- THESEUS (HE transient phenomena, High Sensitivity);

- eASTROGAM ( $200 \mathrm{keV-2} \mathrm{GeV,} \mathrm{High} \mathrm{Sensitivity);}$

- The GAMMA-400 gamma-ray telescope (high angular and energy resolutions);

- The Large Hadron Collider (LHC) (the world's largest and most powerful particle accelerator);

- The BICEP (Background Imaging of Cosmic Extragalactic Polarization) and the Keck Array.

A discussion of these experiments can be found in Giovannelli \& Sabau-Graziati (2018b, and the references therein).

\subsection{Small experiments}

In this short excursion about the tools necessary for an advance of our knowledge of the physics of the Universe, we cannot omit the extreme importance of small experiments, like those Space-based: small-, mini-, micro-, nano-, and cube-satellites, and those Ground-based: smalltelescope, and Robotic-telescopes. 
A discussion on the importance of small experiments for the advancement of knowledge of our Universe can be found in Giovannelli \& Sabau-Graziati (2018b, and the references therein).

Just for giving to the reader a short panorama about the many small ground- and space-based experiments, not necessarily autonomous, we list the following:

- MITSuME (Multicolor Imaging Telescope for Survey and Monstrous Explosions);

- The CHASE (CHilean Automatic Supernova sEarch);

- The Russian global network of telescopes robot MASTER;

- MASTER-Amur, Russian Federation;

- MASTER-Tunka, Russian Federation;

- MASTER-Ural, Russian Federation;

- MASTER-Kislovodsk, Russian Federation;

- MASTER-SAAO, South Africa;

- MASTER-IAC, Spain;

- MASTER-OAFA, Argentina;

- MASTER-Progenitor, Russian Federation.

- Very small satellites for multifreqyency astrophysics.

A discussion of these experiments can be found in Giovannelli \& Sabau-Graziati (2017, and the references therein).

\section{The use of wisdom in Physics}

With the experiments previously listed, we are going to know better the composition and the laws governing our Universe. But for this purpose it is much better the use of wisdom besides the knowledge of the physics.

A rather exhaustive excursion about the use of wisdom in Physics has been reported by Giovannelli \& Sabau-Graziati (2017, and the references therein). Below we show a couple of examples on the use of wisdom in physics.

\subsection{Transient accreting sources}

$\mathrm{X}$-ray/Be systems are formed by a compact star and an optical star. Obviously there is a mutual influence between the two stars. Low-energy (LE) processes influence high-energy (HE) processes and vice versa. Never confuse the effect with the cause. There is a general law in the Universe: Cause and Effect. The Cause generates an Effect and NOT vice versa!

Time-lag between HE events and LE events in disk-fed accreting X-ray binaries (XRBs) has been noted in many systems, but the trigger of the work resulted in a model for explaining in general such a phenomenon (Bisnovatyi-Kogan \& Giovannelli, 2017) was given by Giovannelli \& SabauGraziati (2011) who noted a systematic delay between the relative enhancement in luminosity of 
the optical Be star - occurring at the periastron passage of the neutron star - and the subsequent X-ray flare in the system HDE 245770/A 0535+26. The model for such a system was developed and corroborated by many events (Giovannelli, Bisnovatyi-Kogan \& Klepnev, 2013: GBK13), and later by events reported in Giovannelli et al. (2015) where also a relationship between $\Delta \mathrm{V}_{\text {mag }}$ of the optical star at the periastron and X-ray intensity $\left(\mathrm{I}_{\mathrm{X}}\right)$ of the 8-day delayed flare was produced.

Briefly, the model based on an accretion disk geometrically thin and optically thick without advection (Shakura \& Sunyaev, 1973; Bisnovatyi-Kogan, 2002) is the following: in the vicinity of periastron the mass flux $\dot{M}$ increases (depending on the activity of the Be star) between $\approx 10^{-8}$ and $\approx 10^{-7} \mathrm{M}_{\odot} \mathrm{yr}^{-1}$. The outer part of the accretion disk becomes hotter, therefore the optical luminosity $\left(\mathrm{L}_{\mathrm{opt}}\right)$ increases. Due to large turbulent viscosity, the wave of the large mass flux is propagating toward the neutron star, thus the $\mathrm{X}$-ray luminosity $\left(\mathrm{L}_{\mathrm{x}}\right)$ increases due to the appearance of a hot accretion disk region and due to the accretion flow channeled by the magnetic field lines onto magnetic poles of the neutron star. The time-delay $\tau$ is the time between the optical and X-ray flashes appearance.

It is right to remind that the mechanism proposed by GBK13 for explaining the X-ray-optical delay in A $0535+26 /$ HDE 245770 is based on an enhanced mass flux propagation through the viscous accretion disk. This mechanism, known as UV-optical delay (the delay of the EUV flash with respect to the optical flash) was observed and modeled for cataclysmic variables (e.g. Smak, 1984; Lasota, 2001). Time delays have been detected also in several other X-ray transient binaries. This is the reason that urged Bisnovatyi-Kogan \& Giovannelli (2017) to generalize the aforementioned model, developed for the particular case of A 0535+26/HDE 245770 (Flavia' star). This general model provides the formula (4.1) of the time delay in transient cosmic accreting sources:

$$
\tau=6.9 \frac{\mathrm{m}^{2 / 3} \dot{\mathrm{m}}^{1 / 15}}{\alpha^{4 / 5}\left(\mathrm{~T}_{4}\right)^{28 / 15}}
$$

where:

$\mathrm{m}=\mathrm{M} / \mathrm{M}_{\odot} ; \quad \dot{\mathrm{m}}=\dot{\mathrm{M}} /\left(10^{-8} \mathrm{M}_{\odot} / \mathrm{yr}\right) ; \quad \mathrm{T}_{4}=\mathrm{T}_{0} / 10^{4} \mathrm{~K} ; \quad \alpha=$ viscosity, and

$\mathrm{T}_{0}=$ maximum temperature in optics.

By using this formula it is possible to obtain an excellent agreement between the experimental and theoretical delays found in:

- X-ray/Be system A0535+26/HDE245770: $\tau_{\exp } \simeq 8$ days (GBK13); $\tau_{t h} \simeq 8$ days;

- Cataclysmic variable SS Cygni; $\tau_{\text {exp }}=0.9-1.4$ days (Wheatley, Mauche \& Mattei, 2003); $\tau_{t h} \simeq 1.35$ days;

- Low-mass X-ray binary Aql X-1/V1333 Aql: $\tau_{\exp } \sim 3$ days (Shahbaz et al., 1998); $\tau_{t h} \simeq$ 3.2days

- Black hole X-ray transient GRO J1655-40: $\tau_{\text {exp }} \sim 6$ days (Orosz et al., 1997); $\tau_{t h} \simeq 6.5$ days.

In this general formula the $\alpha$-viscosity parameter plays an important role, and usually it is hard to be determined. However, if the other parameters are known, because experimentally determined, the formula (4.1) can be used for determining $\alpha$, taking into account the experimental delay measured in a certain source. 
This general model for the time-lag for disk-fed accreting XRBs is sketched in Fig. 8.

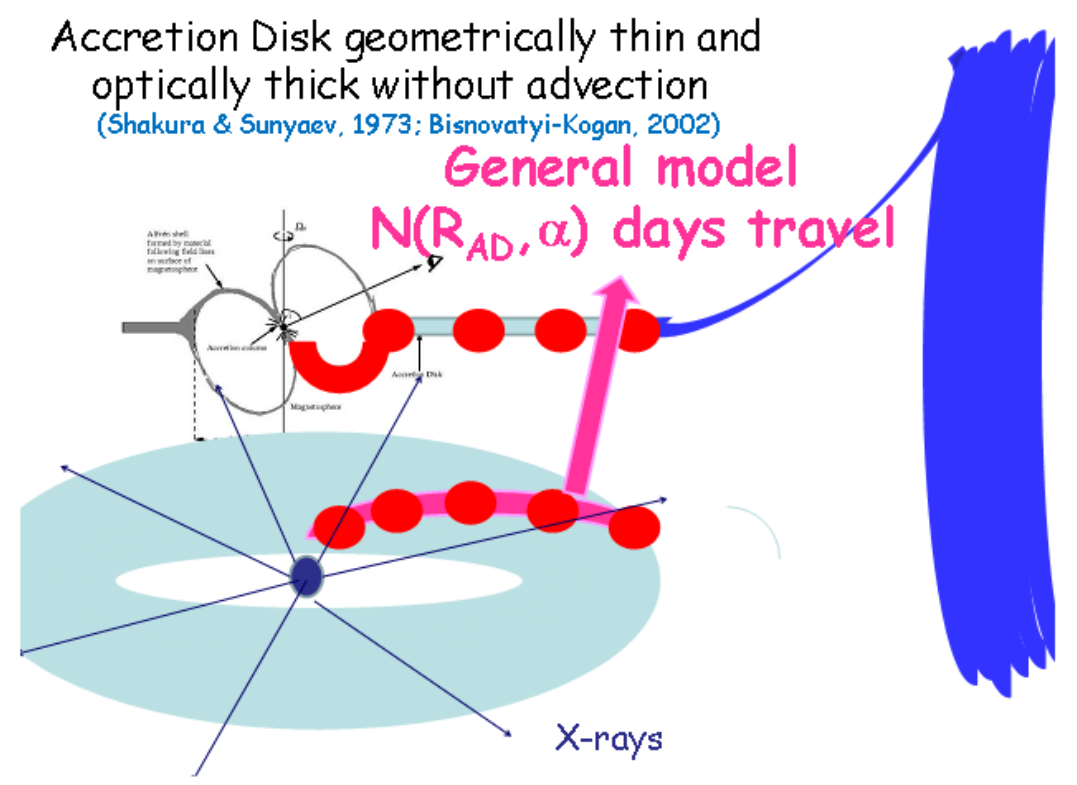

Figure 8: Time-lag general model for disk-fed accreting XRBs (Bisnovatyi-Kogan \& Giovannelli, 2017).

\subsection{Great example of synergy between Astrophysics and History}

Bernd Aschenbach modified Sedov's relation for determining the age of a SNR (Aschenbach, 2016). He used as test the SNR Vela Jr (RX 0852.0-3946) - discovered during the ROSAT AllSky-Survey in X-rays (Aschenbach, 1998) - and he gave an age of $\mathrm{T}_{\text {Aschenbach }} \sim 725 \mathrm{yr}-$ contrary to $\mathrm{T}_{\text {Sedov }} \sim 1714 \mathrm{yr}-$ and a distance of $\sim 386 \mathrm{pc}$.

Historical document (Tatsunokuchi Persecution of Nichiren Daishonin "the Buddha of the last day of the law") supports this result with an exceptional precision: The date of the explosion was 12 September 1271 ( $1 \pm 2$ a.m. - between the hours of the rat and the ox) (Soka Gakkai, 1999). How is it possible to affirm that the explosion of the SN Vela Jr happened in that date with a strong precision?

The answer, indeed, can be found in the writings of Nichiren Daishonin. This buddhist monk presented to the public authority the "Risho Ankoku Ron" (Establishing the Correct Teaching for the peace in the country) three times: a strong and clear critic to the behaviour of authority. For this reason he was persecuted and sentenced to death.

At that moment Nichiren was about to be beheaded, a luminous object (full Moon) shot across the sky, brightly illuminating the surroundings. The executioner fell on his face, his eyes blinded. The soldiers were filled with panic. Terrified, the soldiers called off the execution. This happened on the twelfth day of the ninth month of 1271, between the hours of the rat and the ox (11:00 p.m. to 3:00 a.m.). The event culminated $10^{\circ}$ above the horizon, celestial declination $-46^{\circ}$ (position of Vela Jr). 


\section{The present situation about the knowledge of the physics of our Universe}

Undoubtedly the advent of new generation experiments ground- and space-based have given a strong impulse for verifying current theories, and for providing new experimental inputs for developing a new physics for going, probably, over the standard model (SM). Recent results coming from Active Physics Experiments (APEs) and Passive Physics Experiments (PPEs) have opened such a new path.

An extensive review on the situation about the knowledge of the physics of our Universe has been recently published by Giovannelli \& Sabau-Graziati (2016c). The reader interested is invited to look at that paper. However, we are obliged to discuss a few topics that, in our opinion, could be useful for a better understanding of the open problems still existing in the modern astrophysics.

\subsection{Active physics experiments}

One of the most exciting results from LHC is the detection of the Higgs boson which is often called "the God particle" because it's said to be what caused the "Big Bang" that created our Universe. Matter obtains mass interacting with Higgs field. Thus, if the Higgs Boson is detected, the Standard Model of Physics would be completed.

The Standard Model of particle physics takes quarks and leptons to be fundamental elementary particles, and describes the forces that govern their interactions as mediated through the exchange of further elementary particles. The exchanged particles are photons in the case of the electromagnetic interaction, $\mathrm{W}$ and $\mathrm{Z}$ bosons in the case of the weak interaction, and gluons in the case of the strong interaction. After the discovery of the $\mathrm{W}$ and $\mathrm{Z}$ bosons in the early 1980s, the elucidation of the mechanism by which they acquire mass became an important goal for particle physics. Within the Standard Model the $\mathrm{W}$ and $\mathrm{Z}$ bosons have masses generated via the symmetry breaking Englert-Brout-Higgs-Guralnik-Hagen-Kibble mechanism, proposed in 1964 and giving rise to a massive scalar particle, the Standard Model Higgs boson (Jakobs \& Seez, 2015).

The hunt to Higgs boson started a few years ago with the most powerful accelerators constructed in the world, in particular with the different experiments of the LHC. These experiments can provide information about the first moment of the life of the Universe. LHC is a complementary tool for HE observatories looking directly to the Universe.

The Higgs boson discovery was announced by the ATLAS (A Toroidal LHC ApparatuS) and CMS (Compact Muon Solenoid) collaborations on 4th July 2012. Evidence for a new particle with the mass of about $125 \mathrm{GeV}$ and the properties of the Standard Model Higgs boson.

From ATLAS results, a $5.0 \sigma$ excess at $\sim 126.5 \mathrm{GeV}$ has been detected. This value is compatible with the expected mass of Higg's boson (Gianotti, 2012; Aad et al., 2012). The Compact Muon Solenoid (CMS) experiment at LHC detected a new boson at $125.3 \pm 0.6 \mathrm{GeV}$ with $4.9 \sigma$ significance (Incandela, 2012; Chatrchyan et al. - The CMS Collaboration, 2012). This result, together with that from ATLAS, if confirmed, would complete the SM of physics.

Thanks to collisions at $13 \mathrm{TeV}$ the experiment Large Hadron Collider beauty (LHCb) at LHC detected a new particle: the Pentaquark. The existence of the pentaquark was theoretically suggested since 1960-ies (Gell-Mann, 1964). Pentaquark gives a new way for the combination of the quarks that are the fundamental constituents of neutrons and protons (Cardini, 2015; Aaij et al., 2015). 


\subsection{Passive physics experiments}

One of the most important questions still open is the search for experimental proof of the inflation. The expansion is thought to have been triggered by the phase transition that marked the end of the preceding grand unification epoch at $\approx 10^{-36} \mathrm{~s}$ after the Big Bang. It is not known exactly when the inflationary epoch ended, but it is thought to have been between $\approx 10^{-33}$ and $\approx 10^{-32} \mathrm{~s}$ after the Big Bang. The experimental proof of the inflation could come from measurements of Cosmic Microwave Background (CMB) polarization. Winstein $(2007,2009)$ discussed the problem of CMB polarization in the following decade.

We know from the theory that linear polarization of the CMB photons is induced via Thomson scattering by quadrupole anisotropy at recombination that occurred at $\mathrm{z} \sim 1100$ corresponding to $\mathrm{t} \sim 1.2 \times 10^{13} \mathrm{~s}$ after the Big Bang. In turn, quadrupole anisotropy is induced by: i) density perturbations (scalar relics of inflation) producing a curl-free polarization vector field (E-modes); ii) gravitational waves (tensor relics of inflation) producing both curl-free and curl-polarization fields (B-modes).

No other sources for a curl-polarization field on the CMB at large angular scales exist. Thus, B-modes are a clear signature of inflation (e.g. de Bernardis, 2014).

Recently the collaboration of the BICEP2 experiment claims the detection of E-mode (Crites et al., 2015) and B-mode polarization of the CMB at at $7.0 \sigma$ significance (Ade et al., 2015). If Bmode polarization would be confirmed, the inflationary model of the Universe would be definitively confirmed. However, big discoveries need big confirmations. For a robust detection of B-modes, independent measurements and precise measurements of polarized foregrounds are mandatory.

Indeed, a key element to the primordial interpretation advanced by the BICEP2 team was excluding an explanation based on polarized thermal dust emission from our galaxy (Bucher, 2015). An independent analysis casts doubt on the BICEP2 claim (Flauger, Hill \& Spergel, 2014). In September 2014 the Planck team published a paper on the level of polarized dust emission measured across the whole sky, and in particular in the BICEP2 field (Planck Collaboration, 2014). This work also extrapolated the polarized dust signal seen in the Planck $353 \mathrm{GHz}$ map (a frequency in the Wien tail of the CMB blackbody where dust dominates) down to $150 \mathrm{GHz}$ and reached the conclusion that the BICEP B mode signal could be entirely explained by polarized dust emission although a primordial B mode contribution could not be ruled out.

However, the theory of inflation is criticized by Ijjas, Steinhardt \& Loeb (2013) after Planck2013 results. They suggest that the origin of the Universe is not the Big Bang, but could be a "bouncing" Universe that does not need the inflation. Membrane-Universes that clashed endlessly could be a "plausible" alternative model for the Universe (Erickson et al., 2007; Steinhardt, Turok \& Starkman, 2008). Cyclic models of the universe have the advantage of avoiding initial conditions problems related to postulating any sort of beginning in time (Ijjas, 2018).

For all these reasons is even more important to find an experimental proof of the Inflation.

\subsection{Confirmation of the Theory of General Relativity}

In the last few years two further experimental results confirmed the validity of the theory of General Relativity (GR theory). 


\subsubsection{Gravitational lenses}

Kochanek (2003) discussed "The whys and hows of finding 10,000 lenses", mentioning the first radio lens survey - the MIT - Green Bank survey (MG) - that found lenses by obtaining Very Large Array (VLA) snapshot images of flux-limited samples of $5 \mathrm{GHz}$ radio sources. The Hubble Space Telescope (HST), and Chandra observations (e.g. Dai \& Kochanek, 2005) showed without any doubt that the gravitational lensing is operating.

Gravitational lensing is widely and successfully used to study a range of astronomical phenomena, from individual objects, like galaxies and clusters, to the mass distribution on various scales, to the overall geometry of the Universe (Williams \& Schechter, 1997). They describe and assess the use of gravitational lensing as "gold standards" in addressing one of the fundamental problems in astronomy, the determination of the absolute distance scale to extragalactic objects, namely the Hubble constant.

Several papers have been published about the strong gravitational lensing (e.g. Tyson, Kochanski \& Dell'Antonio, 1998; Tyson, 2000 and references therein), and the weak gravitational lensing (Wittman et al., 2000). A review on "Gravitational Lenses" have been published by Blandford \& Kochanek (2004). A book on "Gravitational Lensing: Strong, Weak and Micro" was published by Meylan et al. (2006). Winn, Rusin \& Kochanek (2004) reported the most secure identification of a central image, based on radio observations of PMN J1632-0033.

Therefore, a further dowel supports the GR theory.

\subsubsection{Gravitational waves}

The Universe that contains by definition all the matter or all the energy available showed one important event that was possible to be detected on the Earth. This event was a further direct experimental demonstration of the validity of the GR theory. Indeed, on September 14, 2015 at 09:50:45 UTC the two detectors of the Laser Interferometer Gravitational-Wave Observatory (LIGO) simultaneously observed a transient gravitational-wave signal. It matches the waveform predicted by GR theory for the inspiral and merger of a pair of black holes and the ringdown of the resulting single black hole. The signal was observed with a significance $\geq 5.1 \sigma$ (Fig. 9). The source lies at a luminosity distance of $410_{-180}^{+160} \mathrm{Mpc}$ corresponding to a redshift $\mathrm{z}=0.090_{-0.04}^{+0.03}$. In the source frame, the initial black hole masses are $36_{-4}^{+5} \mathrm{M}_{\odot}$ and $29 \pm 4 \mathrm{M}_{\odot}$, and the final black hole mass is $62 \pm 4 \mathrm{M}_{\odot}$ with $3.0 \pm 0.5 \mathrm{M}_{\odot} \mathrm{c}^{2}$ radiated in gravitational waves. All uncertainties define $90 \%$ credible intervals. These observations demonstrate the existence of binary stellar-mass black hole systems. This is the first direct detection of gravitational waves and the first observation of a binary black hole merger (Abbott et al., 2016a).

Abbott et al. (2016b) reported the second observation of a gravitational-wave signal produced by the coalescence of two stellar-mass black holes. The signal, GW151226, was observed by the twin detectors of the LIGO on December 26, 2015 at 03:38:53 UTC. The signal was detected at significance $\geq 5 \sigma$. The inferred source-frame initial black hole masses are $14.2_{-3.7}^{+8.3} \mathrm{M}_{\odot}$ and $7.5 \pm$ $2.3 \mathrm{M}_{\odot}$, and the final black hole mass is $20.8_{-1.7}^{+6.1} \mathrm{M}_{\odot}$. We find that at least one of the component black holes has spin greater than 0.2 . This source is located at a luminosity distance of $440_{-190}^{+180}$ Mpc corresponding to a redshift $\mathrm{z}=0.09_{-0.04}^{+0.03}$. All uncertainties define a $90 \%$ credible interval. 


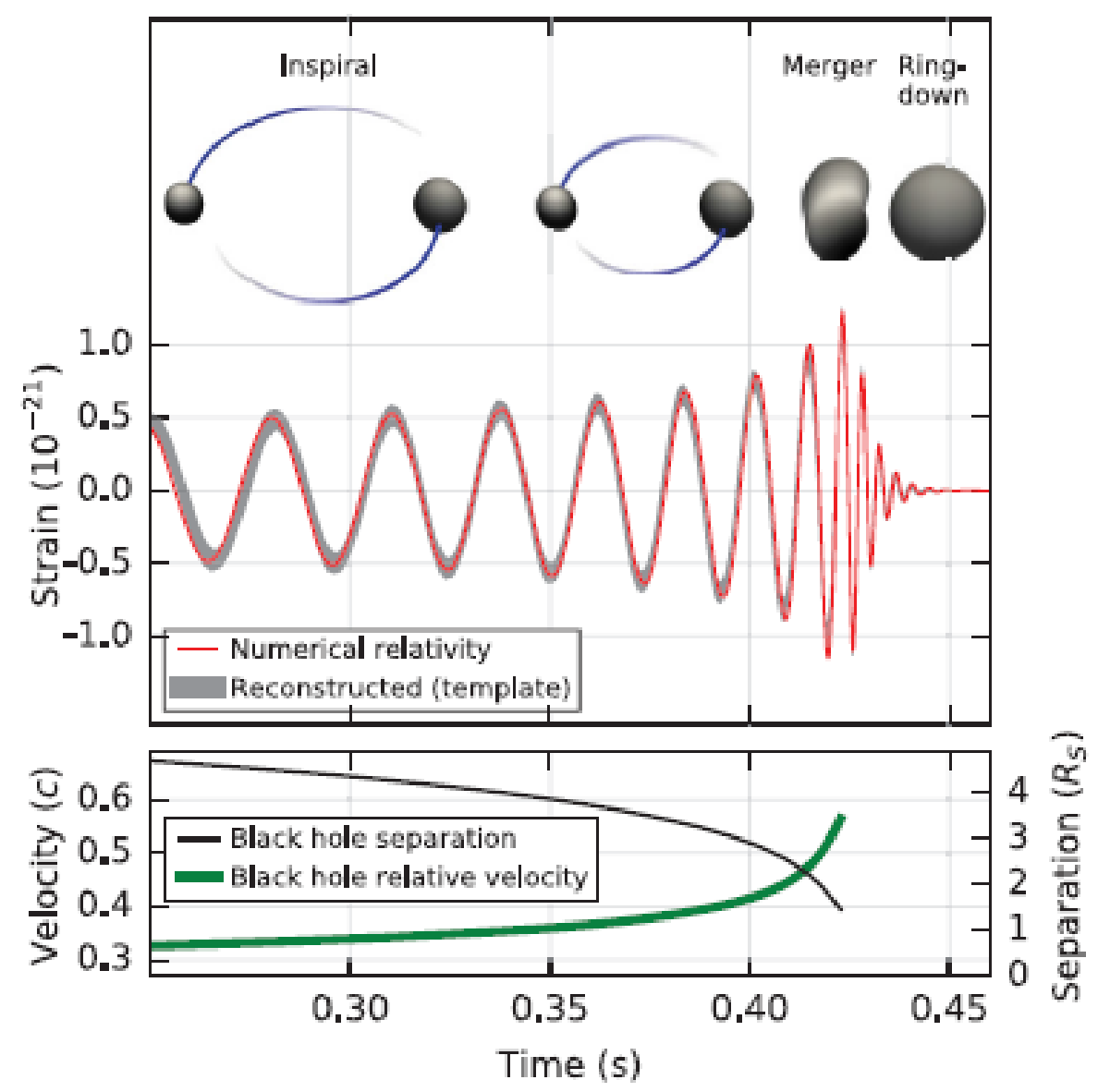

Figure 9: The GW150914 event. Top: estimated gravitational-wave strain amplitude. Bottom: the Keplerian effective black hole separation in units of Schwarzschild radii (adopted from Abbott et al., 2016a).

This second gravitational-wave observation provides improved constraints on stellar populations and on deviations from the GR theory.

For these detections of gravitational waves - first predicted by Einstein 100 years ago - Rainer Weiss, Barry Barish \& Kip Thorne have been awarded the 2017 Nobel prize in physics.

Abbott et al. (2016c) present a possible observing scenario for the Advanced LIGO (aLIGO) and Advanced Virgo gravitational-wave detectors over the next decade, with the intention of providing information to the astronomy community to facilitate planning for multi-messenger astronomy with gravitational waves.

Gravitational waves provide a revolutionary tool to investigate yet unobserved astrophysical objects. Especially the first stars, which are believed to be more massive than present-day stars, might be indirectly observable via the merger of their compact remnants. An interesting paper by Hartwig et al. (2016) developed a self-consistent, cosmologically representative, semi-analytical model to simulate the formation of the first stars. They estimated the contribution of primordial stars to the merger rate density and to the detection rate of the aLIGO. Owing to their higher masses, the remnants of primordial stars produce strong GW signals, even if their contribution in number is relatively small. They found a probability of $\geq 1 \%$ that the current detection GW150914 is of 
primordial origin. The higher masses of the first stars boost their GW signal, and therefore their detection rate. Up to five detections per year with aLIGO at final design sensitivity originate from Pop III BH-BH mergers. Approximately once per decade, we should detect a BH-BH merger that can unambiguously be identified as a Pop III remnant.

On 2017 August 17 the merger of two compact objects with masses consistent with two neutron stars was discovered through gravitational-wave (GW170817), gamma-ray (GRB 170817A), and optical (SSS17a/AT2017gfo) observations. The optical source was associated with the earlytype galaxy NGC 4993 at a distance of just $\sim 40 \mathrm{Mpc}$, consistent with the gravitational-wave measurement, and the merger was localized to be at a projected distance of $\sim 2 \mathrm{kpc}$ away from the galaxy's center (Abbott et al., 2017a,b).

Lipunov et al. (1995) predicted the NS-NS merger at a distance of $\leq 50 \mathrm{Mpc}$ and the possibility of detecting GWs!

This prediction was born by the "Scenario Machine" that describes the evolution of gravimagnetic rotators (Lipunov, 1987; Lipunov, \& Postnov, 1988), and recently commented by Giovannelli (2016).

On August 17, 2017 Multimessenger Astrophysics born! As pioneers of the Multifrequency Astrophysics, we are particularly happy!

\subsection{The accelerating Universe}

The discovery of the accelerating expansion of the Universe is a milestone for cosmology. A very interesting paper about this argument has been published in 2011 by the "Class for Physics of the Royal Swedish Academy of Sciences" as Scientific Background on the Nobel Prize in Physics 2011. In this paper a historical journey about the last century development of cosmology is brilliantly presented.

The discovery in 1998 that the universe is speeding up and not slowing down (Riess et al. 1998; Perlmutter et al. 1999) opened a question about the possibility of having different phases of acceleration and deceleration of the Universe along its life. Turner \& Riess (2002) from observations of SN 1997ff at $\mathrm{z} \sim 1.7$ favor the accelerating universe interpretation and provide some direct evidence that the universe was once decelerating. They show that the strength of this conclusion depends upon the nature of the dark energy causing the present acceleration. Only for a cosmological constant is the SNe evidence definitive. Using a new test which is independent of the contents of the universe, they show that the $\mathrm{SN}$ data favor recent acceleration $(\mathrm{z}<0.5)$ and past deceleration $(\mathrm{z}>0.5)$.

Nielsen, Guffanti \& Sarkar (2016) found marginal evidence for cosmic acceleration from type Ia Supernovae. On the contrary, Haridasu et al. (2017) found that the SN data alone indicate an accelerating Universe at more than $4.56 \sigma$ confidence level.

The solution of the problem of the expanding Universe will be probably come from EUCLID, which is a European Space Agency medium-class mission selected for launch in 2020 within the cosmic vision 2015-2025 program. The main goal of Euclid is to understand the origin of the accelerated expansion of the universe. Euclid will explore the expansion history of the universe and the evolution of cosmic structures by measuring shapes and redshifts of galaxies as well as the distribution of clusters of galaxies over a large fraction of the sky. However, although the main 
driver for Euclid is the nature of dark energy, Euclid science covers a vast range of topics, from cosmology to galaxy evolution to planetary research (Amendola et al., 2018).

\subsection{The Big Bang Nucleosynthesis theory has been proved}

The Big Bang Nucleosynthesis (BBN) theory predits the presence of a fixed content of light elements, the temperature of the Universe inversely proportional to the typical distance between galaxy clusters: $\mathrm{T}=\mathrm{T}(0)(1+\mathrm{z})$, and the $\mathrm{CMB}$ radiation temperature of $\sim 2.7 \mathrm{~K}$ (for details see the paper by Giovannelli \& Sabau-Graziati, 2016c).

\subsection{Is the Universe Flat?}

One of the most critical points about our Universe is the problem of its flatness. The present state of the cosmological tests is illustrated in Fig. 10.

\section{(Schuecker, P., 2005)}

(de Bernardis, P. et al., 2000)

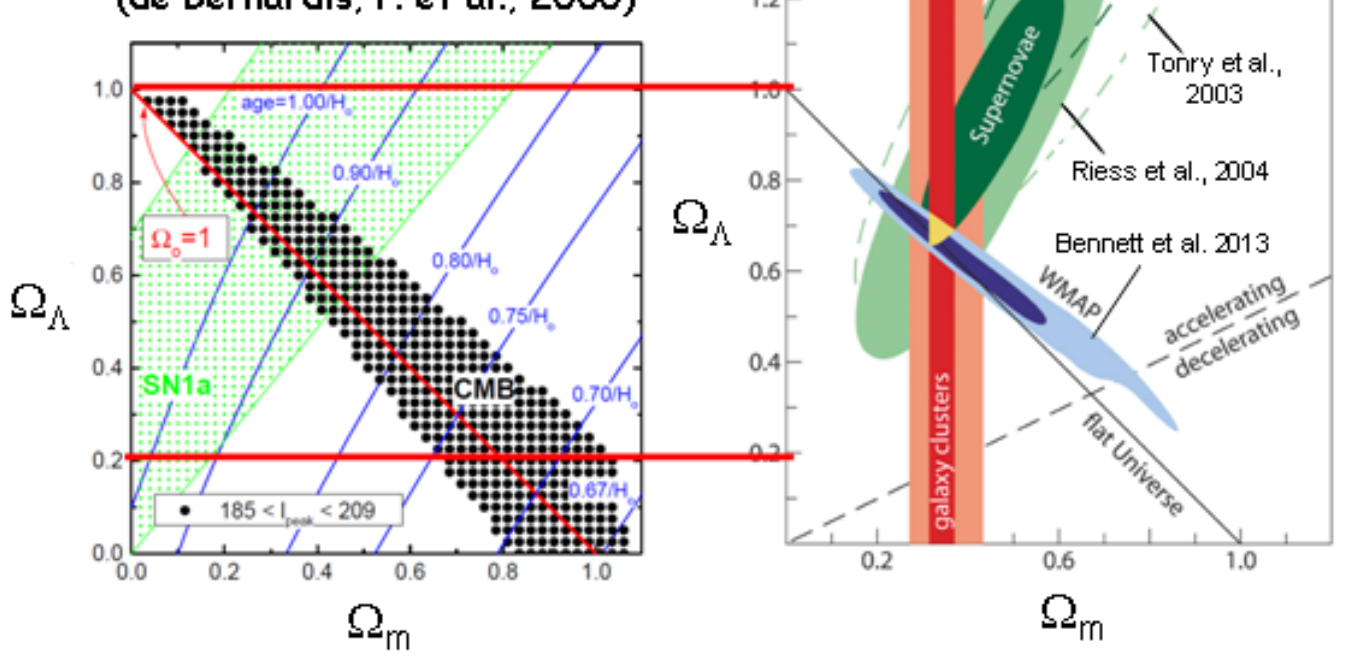

Figure 10: Constraints of cosmological parameters (after de Bernardis et al., 2000; Schuecker, 2005, Bennett et al., 2013).

The left panel of Fig. 10 shows the results obtained with the BOOMERanG (Balloon Observations Of Millimetric Extragalactic Radiation and Geomagnetics) experiment (de Bernardis et al., 2000). They are fully consistent with a spatially flat Universe. The right panel of Fig. 10 shows the combination of the likelihood contours obtained with three different observational approaches: i) type-Ia SNe (Tonry et al., 2003; Riess et al. 2004); ii) CMB (Spergel et al. 2003; Bennett et al. 2013); iii) galaxy clusters (Schuecker et al. 2003; Schuecker, 2005). One can see that the cosmic matter density is close to $\Omega_{\mathrm{m}}=0.3$, and that the normalized cosmological constant is around $\Omega_{\Lambda}=0.7$. This sums up to unit total cosmic energy density and suggests a spatially flat universe. However, the density of cosmic matter growths with redshift like $(1+\mathrm{z})^{3}$ whereas the density $\rho_{\Lambda}$ related to the cosmological constant $\Lambda$ is independent of $z$. The final results from WMAP (Bennett 
et al., 2013) shows a little misalignment with the line of "flat Universe". Thus it is necessary to be careful in the conclusions.

\subsection{Hubble Constant}

The Hubble constant $\left(\mathrm{H}_{0}\right)$ is one of the most important numbers in cosmology because it is needed to estimate the size and age of the universe. The important problem of determination of $\mathrm{H}_{0}$ value is one of the most exciting. Indeed, in the literature it is possible to find many determinations coming from different experiments using different methods. However, it is very complicated to obtain a true value for $\mathrm{H}_{0}$. It is necessary to have two measurements: i) spectroscopic observations that reveal the galaxy's redshift, indicating its radial velocity; ii) the galaxy's precise distance from Earth (and this is the most difficult value to determine).

For a discussion about $\mathrm{H}_{0}$ see the paper by Giovannelli \& Sabau-Graziati (2014a and the references therein).

However, Riess et al. (2011) with the HST determined a value of $\mathrm{H}_{0}=73.8 \pm 2.4 \mathrm{~km} \mathrm{~s}^{-1}$ $\mathrm{Mpc}^{-1}$. This value agrees with the WMAP results: $\mathrm{H}_{0}=71.0 \pm 2.5 \mathrm{~km} \mathrm{~s}^{-1} \mathrm{Mpc}^{-1}$ (Komatsu et al., 2011). Bennett et al. (2014) discussed the progress occurred in recent years for determining the Hubble constant: results coming from the cosmic distance ladder measurements at low redshift and $\mathrm{CMB}$ measurements at high redshift. The $\mathrm{CMB}$ is used to predict the current expansion rate of the universe by best-fitting cosmological models. At low redshift baryon acoustic oscillation (BAO) measurements have been used - although they cannot independently determine $\mathrm{H}_{0}$ - for constraining possible solutions and checks on cosmic consistency. Comparing these measurements they found $\mathrm{H}_{0}=69.6 \pm 0.7 \mathrm{~km} \mathrm{~s}^{-1} \mathrm{Mpc}^{-1}$.

Does this determination, finally, close the history about the search of the "true" value of $\mathrm{H}_{0}$ ?

\subsection{Reionization Epoch}

Ground-based observations of the CMB on subdegree angular scales suggest that the gas content of the universe was mostly neutral since recombination at $\mathrm{z} \sim 1000$ until about $\mathrm{z} \sim 100$ (Gnedin, 2000 and the references therein) because earlier reionization would have brought the last scattering surface to lower redshift, smoothing the intrinsic CMB anisotropy. At the same time, we know that the universe is highly ionized, since $\mathrm{z} \approx 5$, from observations of the spectra of quasars with the highest redshifts (e.g. Giallongo et al. 1994). This change of the ionization state of the universe from neutral to highly ionized is called "reionization". How large is the redshift to which the reionization started and stopped is object of strong debate. In our opinion probably it is possible to put a reasonable limit to the epoch of the reionization end $(\mathrm{z} \sim 6)$, looking at the paper by Toshikawa et al. (2012).

An interesting review about The epoch of reionization was published by Zaroubi (2013), and a discussion about this topic can be found in the paper by Giovannelli \& Sabau-Graziati (2016c and the references therein).

Many papers have been published about the problem of reionization. The reionization history of cosmic hydrogen, left over from the big bang, provides crucial fossil evidence for when the first stars and black holes formed in the infant universe (Barkana \& Loeb, 2001). The hyperfine spin-flip transition of neutral hydrogen (HI) at a wavelength of $21 \mathrm{~cm}$ is potentially the most promising probe 
of the cosmic gas before reionization ended. Observations of this line at a wavelength of $21(1+\mathrm{z})$ $\mathrm{cm}$ can be used to slice the universe as a function of redshift $\mathrm{z}$ and obtain a three-dimensional map of the diffuse HI distribution in it (Hogan \& Rees 1979).

The first galaxies that formed at a redshift $\mathrm{z} \sim 20$-30 emitted continuum photons with energies between the Ly- $\alpha$ and Lyman limit wavelengths of hydrogen, to which the neutral universe was transparent except at the Lyman series resonances. As these photons redshifted or scattered into the Ly- $\alpha$ resonance, they coupled the spin temperature of the $21 \mathrm{~cm}$ transition of hydrogen to the gas temperature, allowing it to deviate from the microwave background temperature. Barkana \& Loeb (2005) showed that the fluctuations in the radiation emitted by the first galaxies produced strong fluctuations in the $21 \mathrm{~cm}$ flux before the Ly- $\alpha$ coupling became saturated. The fluctuations were caused by biased inhomogeneities in the density of galaxies, along with Poisson fluctuations in the number of galaxies. Observing the power spectra of these two sources would probe the number density of the earliest galaxies and the typical mass of their host dark matter halos. The enhanced amplitude of the $21 \mathrm{~cm}$ fluctuations from the era of Ly- $\alpha$ coupling improves considerably the practical prospects for their detection.

Indeed, Barkana (2018) shows that 21-cm cosmology can be used as a dark-matter probe. In fact the recent detection of the global 21-cm spectrum (Bowman et al., 2018) reveals a stronger absorption than the maximum predicted by existing models, at a confidence level of $3.8 \sigma$. This allows Barkana to state that this absorption can be explained by the combination of radiation from the first stars and excess cooling of the cosmic gas induced by its interaction with dark matter. His analysis indicates that the spatial fluctuations of the $21-\mathrm{cm}$ signal at cosmic dawn could be an order of magnitude larger than previously expected and that the dark-matter particle is no heavier than several proton masses, well below the commonly predicted mass of weakly interacting massive particles. He also confirms that dark matter is highly non-relativistic and at least moderately cold, and primordial velocities predicted by models of warm dark matter are potentially detectable.

Feng \& Holder (2018) discuss the enhanced global signal of neutral hydrogen due to excess radiation at cosmic dawn incorporating a possible radio background at early times. They find that the global $21 \mathrm{~cm}$ signal shows a much stronger absorption feature, which could enhance detection prospects for future $21 \mathrm{~cm}$ experiments. In the introduction of their paper they report the list of experiments devoted to the Epoch of Reionization (EoR) and the relative references.

Madau (2018), under the basic assumption that the $21-\mathrm{cm}$ signal is activated by young, metalpoor stellar systems, shows that the EDGES (Experiment to Detect the Global Epoch of reionization Signature: Bowman \& Rogers, 2010) signal is consistent with an extrapolation of the evolving galaxy UV luminosity density measured at $4 \lesssim \mathrm{z} \lesssim 9$ by deep HST observations. Models of accelerated evolution where the UV luminosity density declines rapidly at $\mathrm{z} \gtrsim 10$ are unable to provide the needed Ly- $\alpha$ coupling strength at $\mathrm{z} \lesssim 20$. If his analysis is correct and the EDGES results are confirmed, galaxy light appears to have built up at a surprisingly steady rate over the first Gyr of cosmic history; in other words, a substantially enhanced star formation rate density or exotic luminous sources do not seem to characterize the epoch of first light.

Salvador Solé, Manrique \& Canales (2018) review the present knowledge of two fundamental processes in cosmology: galaxy formation and reionization, providing a huge list of references. They conclude that our ignorance on the Pop III star IMF (Initial Mass Function) still leads to a small degeneracy in the allowed solutions as two different solutions, one with single and the other 
with double reionization, are still possible. However, the rapidly growing observational data on the high-z Universe, coming from the CMB anisotropies and $21 \mathrm{~cm}$ line experiments as well as the observation of very distant LAEs (Ly- $\alpha$ Emitters) could soon leave that degeneracy.

\subsection{Extragalactic Background Light}

The intergalactic space is filled with the light produced by all the stars and accreting compact objects that populated the observable Universe throughout the whole cosmic history. This relic cosmic background from IR to UV is called the diffuse Extragalactic Background Light (EBL), long before known as DEBRA (Diffuse Extragalactic Background RAdiation) (Ressell \& Turner, 1990).

Direct measurements of the EBL are difficult due to bright local foregrounds. A powerful approach for probing these diffuse radiation fields in the UV to far-IR bands is through $\gamma-\gamma$ absorption of high-energy photons. Actually pair production $\left(\mathrm{e}^{+} \mathrm{e}^{-}\right)$against EBL photons with wavelengths from ultraviolet to infrared is effective at attenuating $\gamma$-rays with energy above $\sim 10 \mathrm{GeV}$. This process introduces an attenuation in the spectra of $\gamma$-ray sources above a critical energy (e.g. Costamante, 2012; Buson, 2014).

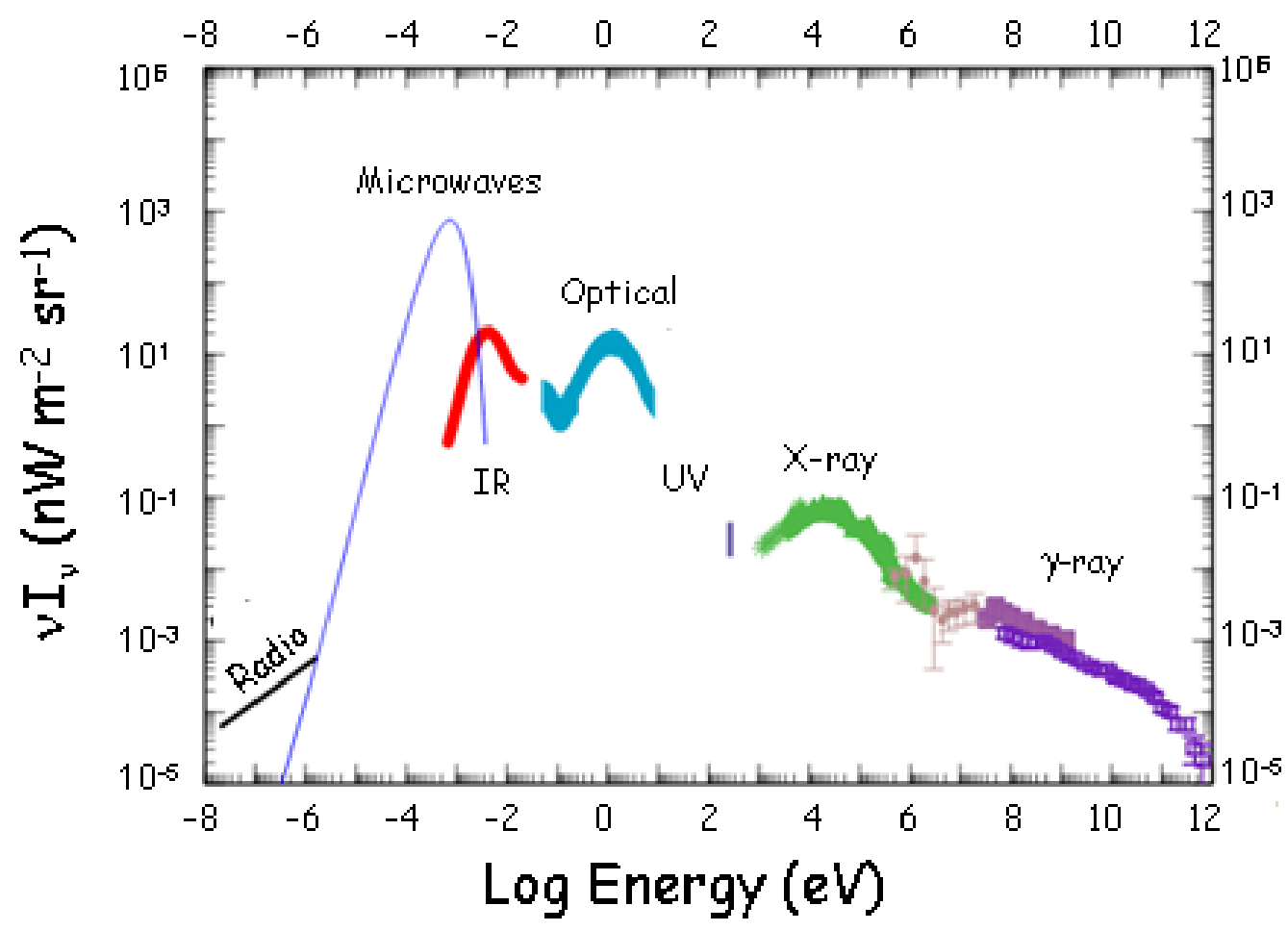

Figure 11: Intensity of the extragalactic background $\left(v \mathrm{I}_{v}\right.$ in units of $\left.\mathrm{nW} \mathrm{m} \mathrm{m}^{-2} \mathrm{sr}^{-1}\right)$ as a function of the energy (after Cooray, 2016).

The last decade has been foreboding of a full coverage of the HE-VHE $\gamma$-ray energy band, thanks to the many ground- and space-based high sensitivity experiments. Thus it has been possible to collect a large amount of data from many extragalactic emitters at high redshift (e.g. Costamante, 2012). Thanks to measurements of the quasar 3C $279(\mathrm{z} \simeq 0.54)$ obtained with the MAGIC 
experiment (Magic Collaboration, 2008), and with the many sources at high redshift, including Gamma Ray Bursts (GRBs) measured with the FERMI observatory (Abdo et al., 2010), it has been demonstrated that the Universe is more transparent to $\gamma$-rays than before believed (Coppi \& Aharonian, 1997).

Cooray (2016) reviewed the Extragalactic Background Light Measurements and Applications. This review covers the measurements related to the extragalactic background light intensity from $\gamma$-rays to radio in the electromagnetic spectrum over 20 decades in wavelength. Figure 11 shows such EBL measurements that updated those reported by Ressell \& Turner (1990). It is important to remark that the numerous measurements in the range of the VHE $\gamma$-rays $(\log \mathrm{E} \approx 9-13 \mathrm{eV})$ have filled the zone where no measurements or only upper limits were available in the 1990-ies.

The CMB remains the best measured spectrum with an accuracy better than $1 \%$. Durrer (2015) in her interesting review describes the discovery of the cosmic microwave background radiation in 1965 and its impact on cosmology in the 50 years that followed.

Henry et al. (2015) discussed the diffuse cosmic background radiation in the Galaxy Evolution Explorer far-ultraviolet (FUV, 1300-1700). They deduced that the UV diffuse cosmic background radiation originates only partially in the dust-scattered radiation of FUV-emitting stars: the source of a substantial fraction of the FUV background radiation remains a mystery. They also discussed about our limited knowledge of the cosmic diffuse background at ultraviolet wavelengths shortward of $\operatorname{Ly} \alpha$ - it could be that a "second component" of the diffuse FUV background persists shortward of the Lyman limit and is the cause of the reionization of the universe.

\subsection{Star Formation Rate History}

In his splendid review, Robert C. Kennicutt, Jr. (1998) discussed the observations of star formation rates (SFRs) in galaxies that provide vital clues to the physical nature of the Hubble sequence and showing that these observations are key probes of the evolutionary histories of galaxies. Kennicutt \& Evans (2012) reviewed the progress over the previous decade in observations of large-scale star formation, with a focus on the interface between extragalactic and Galactic studies. Methods of measuring gas contents and star-formation rates have been discussed, and updated prescriptions for calculating star-formation rates were provided. They reviewed relations between star formation and gas on scales ranging from entire galaxies to individual molecular clouds.

The key dynamical processes involved in star formation - turbulence, magnetic fields, and self-gravity - are highly nonlinear and multidimensional. Therefore, it is extremely difficult a complete quantitative description of the physics involved in the process of star formation. McKee \& Ostriker (2007) attempted to review the theory of star formation. For this reason they divided star formation into large-scale and small-scale regimes and reviewed each in turn. Large scales range from galaxies to giant molecular clouds (GMCs) and their substructures. Important problems include how GMCs form and evolve, what determines the star formation rate (SFR), and what determines the initial mass function (IMF) have been discussed. Small scales range from dense cores to the protostellar systems they beget. They discussed formation of both low- and high-mass stars, including ongoing accretion. The development of winds and outflows is increasingly well understood, as are the mechanisms governing angular momentum transport in disks. However, they concluded that a comprehensive theory of star formation will be tested by the next generation of telescopes. 


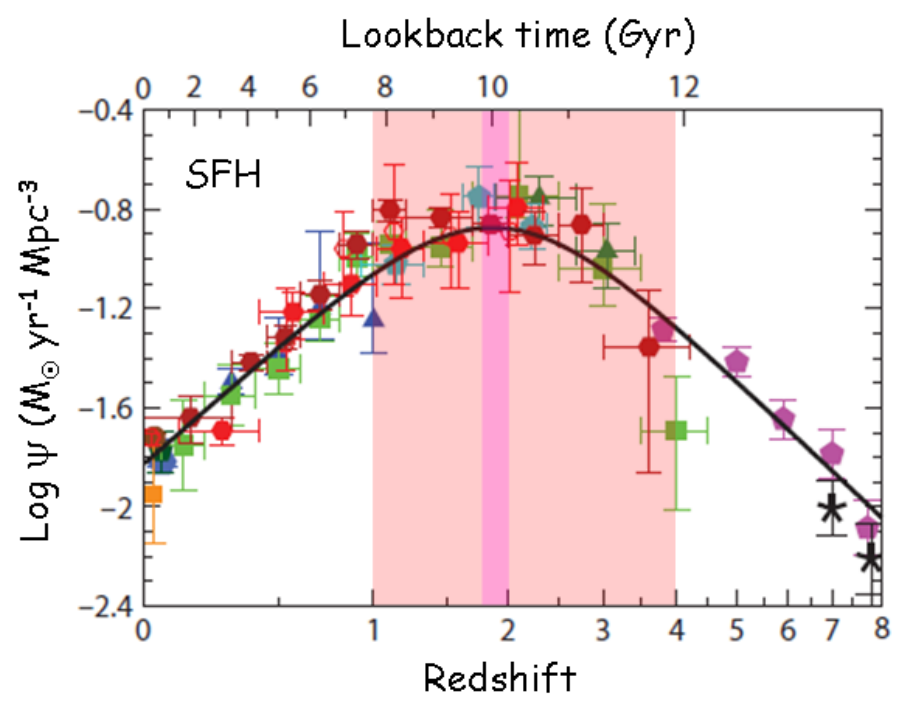

Figure 12: The history of star formation from FUV+IR rest-frame measurements (adapted from Madau \& Dickinson, 2014).

Fumagalli et al. (2012) investigated the evolution of the $\mathrm{H}_{\alpha}$ equivalent width, $\mathrm{EW}\left(\mathrm{H}_{\alpha}\right)$, with redshift and its dependence on stellar mass, using the first data from the 3D-HST survey, a large spectroscopic Treasury program with the HST-WFC3. Combining these data with those from ground-based telescopes, they found that at all masses the characteristic $\mathrm{EW}\left(\mathrm{H}_{\alpha}\right)$ is decreasing towards the present epoch, and that at each redshift the $\mathrm{EW}\left(\mathrm{H}_{\alpha}\right)$ is lower for high-mass galaxies.

The cosmic history of star formation, heavy element production, and reionization of the Universe from the cosmic "dark ages" to the present epoch has been discussed in the review paper by Madau \& Dickinson (2014). A consistent picture is emerging, whereby the star-formation rate density peaked approximately 3.5 Gyr after the Big Bang, at $\mathrm{z} \approx 1.9$, and declined exponentially at later times, with an e-folding timescale of 3.9 Gyr. Half of the stellar mass observed today was formed before a redshift $\mathrm{z}=1.3$. About $25 \%$ formed before the peak of the cosmic star-formation rate density, and another $25 \%$ formed after $\mathrm{z}=0.7$. Less than $\sim 1 \%$ of today's stars formed during the epoch of reionization.

However, these results were already largely discussed and presented by Hopkins \& Beacom (2006), and later by Madau \& Dickinson (2014) and summarized in the Fig. 12, where the cosmic Star Forming History (SFH) from UV and IR data are shown. The light-red rectangle marks the range of redshift where the star formation density had the maximum whose peak is at $\mathrm{z} \sim 1.9 \pm 0.1$ and marked with the light fuchsia rectangle. The black line is the best-fitting function:

$$
\Psi(z)=0.015 \frac{(1+z)^{2 / 7}}{1+[(1+z) / 2.9]^{5.6}}
$$

being $\Psi(z)$ expressed in $\mathrm{M}_{\odot} \mathrm{yr}^{-1} \mathrm{Mpc}^{-3}$.

This will be better understood when the supernova rate density evolution, the ranges of stellar masses leading to core-collapse and type Ia supernovae, and the antineutrino and neutrino backgrounds from core-collapse supernovae will be known thanks to the next generation experiments both ground- and space-based. 


\subsection{Gamma Ray Bursts}

Long discussions about Gamma-ray bursts (GRBs) can be found in numerous publications. A list of these can be found in GSG2004 and in Giovannelli \& Sabau-Graziati (2016c).

Important implications on the origin of the highest redshift GRBs are coming from the detection of the GRB 080913 at $\mathrm{z}=6.7$ (Greiner et al., 2009), GRB 090423 at $\mathrm{z} \sim 8.2$ (Tanvir et al., 2009), and GRB 090429B at $\mathrm{z}=9.4$ (Cucchiara et al., 2011). This means that really we are approaching to the possibility of detecting GRBs at the end of Dark Era, where the first Pop III stars appeared. Izzo et al. (2010) discussed successfully a theoretical interpretation of the GRB 090423 within their fireshell model.

Wang \& Dai (2009) studied the high-redshift star formation rate (SFR) up to $\mathrm{z} \simeq 8.3$ considering the Swift GRBs tracing the star formation history and the cosmic metallicity evolution in different background cosmological models including $\Lambda \mathrm{CDM}$, quintessence, quintessence with a time-varying equation of state and brane-world models. $\Lambda \mathrm{CDM}$ model is the preferred which is however compared with other results.

Although big progress has been obtained in the last few years, GRBs theory needs further investigation in the light of the experimental data coming from old and new satellites, often coordinated, such as BeppoSAX or BATSE/RXTE or ASM/RXTE or IPN or HETE or INTEGRAL or SWIFT or AGILE or FERMI or MAXI. Indeed, in spite of thousands papers appeared in the literature since the discovery of GRBs, the problem of their energy emission is still elusive: i) what is jet's composition? (kinetic or magnetic?); ii) where is dissipation occurring? (photosphere? deceleration radius?); iii) how is radiation generated? (synchrotron, Inverse Compton, hadronic?) (Zhang, 2013a,b).

Kumar \& Zhang (2015) in a review paper The Physics of Gamma-Ray Bursts \& Relativistic Jets discussed what we have learned about relativistic collisionless shocks and particle acceleration from GRB afterglow studies, and the current understanding of radiation mechanism during the prompt emission phase. They pointed out how these explosions may be used to study cosmology, e.g. star formation, metal enrichment, reionization history, as well as the formation of first stars and galaxies in the Universe.

The idea that GRBs could be associated to gravitational waves (GWs) emission is now popular. Indeed, short GRBs are believed to be produced by the mergers of either double NSs or NS-BH binaries (Nakar, 2007) and the recent observation of a kilonova associated with GRB130603B (Tanvir et al., 2013; Berger, Fong \& Chornock, 2013) lends support to this hypothesis. In a recent review, D'Avanzo (2015) discussed the observational properties of short GRBs and showed how the study of these properties can be used as a tool to unveil their elusive progenitors and provide information on the nature of the central engine powering the observed emission. The increasing evidence for compact object binary progenitors makes short GRBs one of the most promising sources of gravitational waves for the forthcoming Advanced LIGO/Virgo experiments. This idea obtained recently its experimental verification with the detection of GW 170817 event associated with the GRB 170817A (Abbott et al,, 2017a,b).

Thanks to the NASA's Swift satellite we assisted to ten years of amazing discoveries in time domain astronomy. Its primary mission is to chase GRBs. The list of major discoveries in GRBs and other transients includes the long-lived X-ray afterglows and flares from GRBs, the first ac- 
curate localization of short GRBs, the discovery of GRBs at high redshift ( $\mathrm{z}>8$ ) (Gehrels \& Cannizzo, 2015). And essentially thanks to these discoveries we are now closer to understand the real nature of GRBs.

The recent review by Bernardini (2015) discussed how the newly-born millisecond magnetars can compete with black holes as source of the GRB power, mainly with their rotational energy reservoir. They may be formed both in the core-collapse of massive stars, and in the merger of neutron star or white dwarf binaries, or in the accretion-induced collapse of a white dwarf, being thus a plausible progenitor for long and short GRBs, respectively.

Ghirlanda et al. (2015) discussed about the apparent separation of short and long GRBs in the hardness ratio vs duration plot. This separation has been considered as a direct evidence of the difference between these two populations. The origin of this diversity, however, has been only confirmed with larger GRB samples but not fully understood. They concluded that short and long GRBs have similar luminosities and different energetics (i.e. proportional to the ratio of their average durations). Then, it seems that the results are pointing toward the possibility that short and long GRBs could be produced by different progenitors but the emission mechanism responsible for their prompt emission might be similar.

Piron (2016) in his review discussed the updated knowledge of GRBs at very high energies. Their huge luminosities involve the presence of a newborn stellar-mass black hole emitting a relativistic collimated outflow, which accelerates particles and produces non-thermal emissions from the radio domain to the highest energies. He reviewed recent progresses in the understanding of GRB jet physics above $100 \mathrm{MeV}$, based on Fermi observations of bright GRBs, and discussed the physical implications of these observations and their impact on GRB modeling.

Recently Arnon Dar (2017) proposed again to the attention of the international community his Cannonball (CB) model for explaining the physics of GRBs. In the CB model, GRBs and their afterglows are produced by the interaction of bipolar jets of highly relativistic plasmoids (CBs) of ordinary matter with the radiation and matter along their trajectory. Such jetted CBs are presumably ejected in accretion episodes of fall-back material on the newly formed compact stellar object in core-collapse supernovae (SNe) of Type Ic, in merger of compact stellar objects in close binary systems, and in phase transitions in compact stars (Shaviv \& Dar, 1995; Dar, 1997; Dar \& De Rujula, 2000; Dado \& Dar, 2013a). Dado, Dar \& De Rújula (2009) discussed a long series of different SWIFT GRBs, showing that the CB model fits all their broadband light curves. Dado \& Dar (2013b) discussed the jet break in the X-ray afterglow of GRBs that appears to be correlated to other properties of the X-ray afterglow and the prompt gamma ray emission, but the correlations are at odds with those predicted by the conical fireball (FB) model of GRBs (Piran, 1999). On the contrary they are in good agreement, however, with those predicted by the CB model of GRBs.

Finally, Dado \& Dar (2016) discussed on the critical test of gamma-ray bursts theories and demonstrated definitively the validity of the CB model against the popular FB model (Piran, 1999).

Figure 13 shows, as example, the fits of light curves of GRB 060729, GRB 061007, GRB 160625B, and GRB 130427A by using the CB model.

In our opinion the problem of the models for explaining the behaviour of GRBs can be considered, very probably, closed. The CB model is the best in absolute for the description of the physics governing the GRBs. 

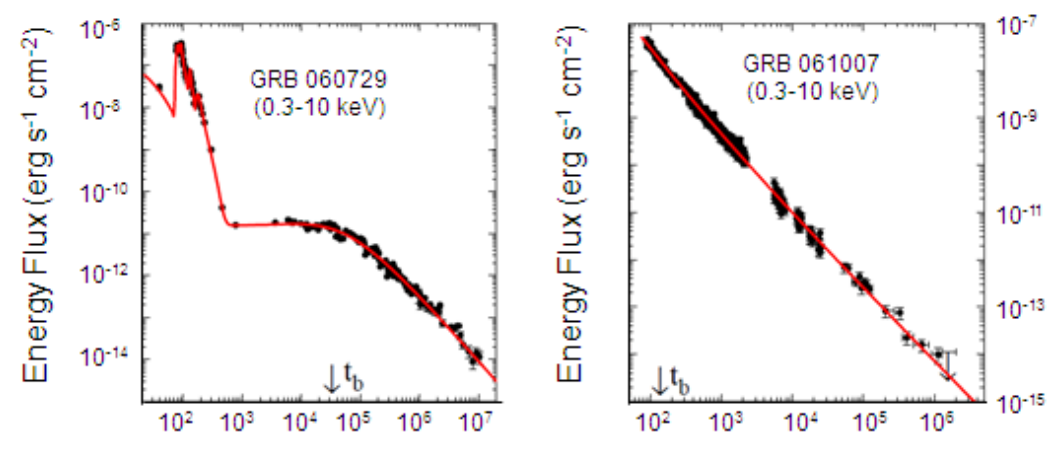

Time since Trigger (s)

Time since Trigger (s)
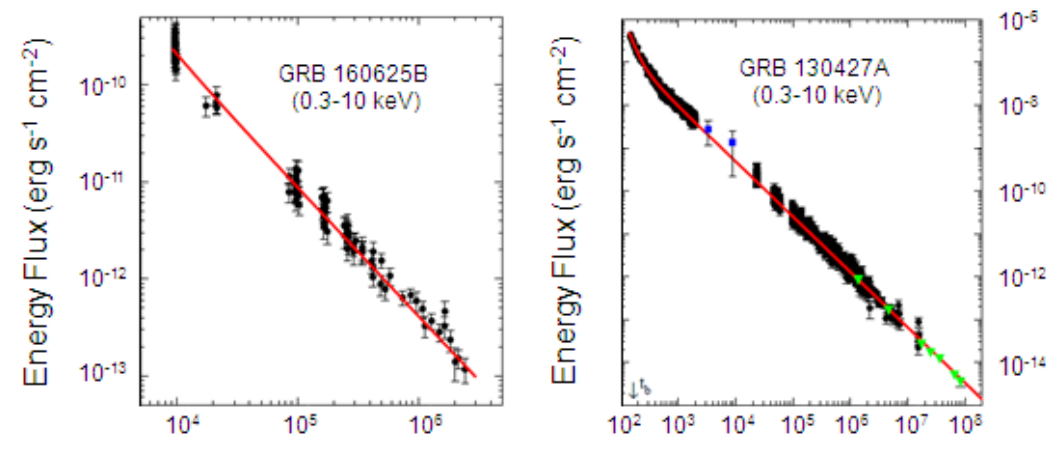

Time since BAT Trigger (s)

Time since BAT Trigger (s)

Figure 13: The 0.3-10keV X-ray light-curve measured with the Swift XRT (Evans et al., 2009), and the comparison between Swift observations and their CB-model description (adapted from Dado \& Dar, 2016) for: top left GRB 060729, top right GRB 061007, bottom left GRB 160625B, bottom right GRB 130427A. This latter figure reports data from different experiments: Swift XRT (black circles), XMM Newton and Chandra (green triangles) (De Pasquale et al., 2016), and the two MAXI data points (blue squares) (Maselli et al., 2014) at $\mathrm{t}=3257 \mathrm{~s}$ and $\mathrm{t}=8821 \mathrm{~s}$. The BAT trigger time is marked with $\mathrm{t}_{\mathrm{b}}$.

\subsection{Magnetars}

A large diversity of neutron stars has been discovered by recent multifrequency observations from the radio band to the X-ray and gamma-ray energy range. Among different manifestation of neutron stars - which include soft gamma repeaters (SGRs), anomalous X-ray pulsars (AXPs), high-B pulsars (HBPs), rotating radio transients (RRATs), central compact objects (CCOs), rotation-powered radio pulsars (RPPs), and X-ray isolated neutron stars (XINSs) (Harding, 2013) - magnetars are strongly magnetized objects with the magnetic field strength of $B=$ $10^{14-15} \mathrm{G}$.

These various manifestations of neutron stars show different characteristics of rotation period $\mathrm{P}$ and its derivative $\dot{\mathrm{P}}$. The measurements of $\mathrm{P}$ and $\dot{\mathrm{P}}$ provide to estimate the dipole magnetic field strength $\mathrm{B}_{\mathrm{d}} \propto \sqrt{\mathrm{P} \dot{\mathrm{P}}}$ and characteristic age $\tau_{\mathrm{c}}=\mathrm{P} / 2 \dot{\mathrm{P}}$.

Enoto (2018) shows in Fig. 14 the P-P diagram, where SGRs and AXPs are collectively called "magnetars" since their slow rotation $(\mathrm{P} \sim 2-12 \mathrm{~s})$ and high period derivatives $\left(\dot{\mathrm{P}} \sim 10^{-13}-10^{-9}\right.$ $\mathrm{s} \mathrm{s}^{-1}$ ) indicate high magnetic fields $\mathrm{B}=10^{14-15} \mathrm{G}$ and young characteristic age $\tau_{\mathrm{c}} \lesssim 10-100 \mathrm{kyr}$. To date, there are 23 known magnetars in the Milky Way and local universe. Figure 15 shows the 


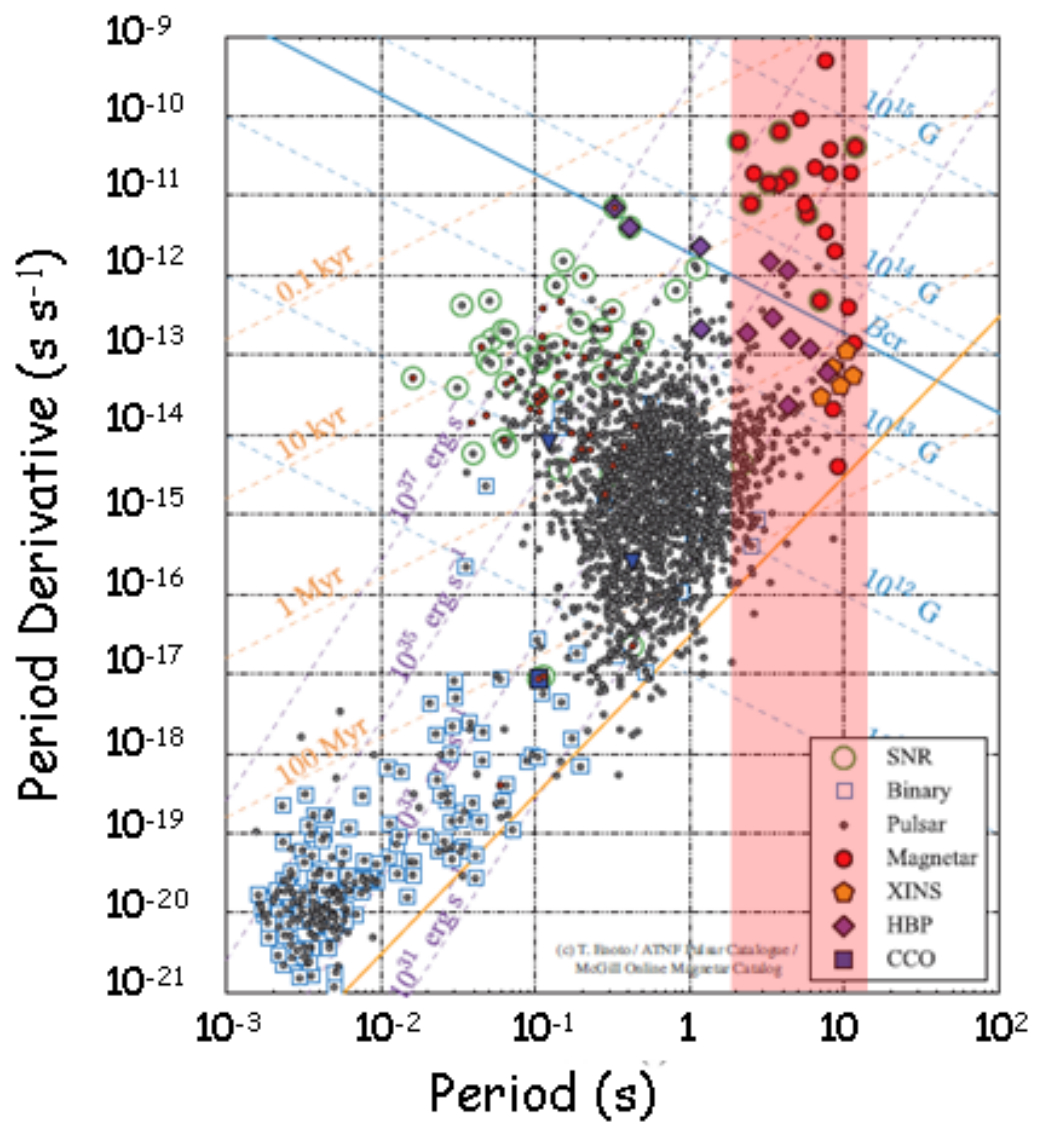

Figure 14: Magnetars and neutron stars on the $\mathrm{P}-\dot{\mathrm{P}}$ diagram. The light-red rectangle limits the range of magnetars periods (adapted from Enoto, 2018).

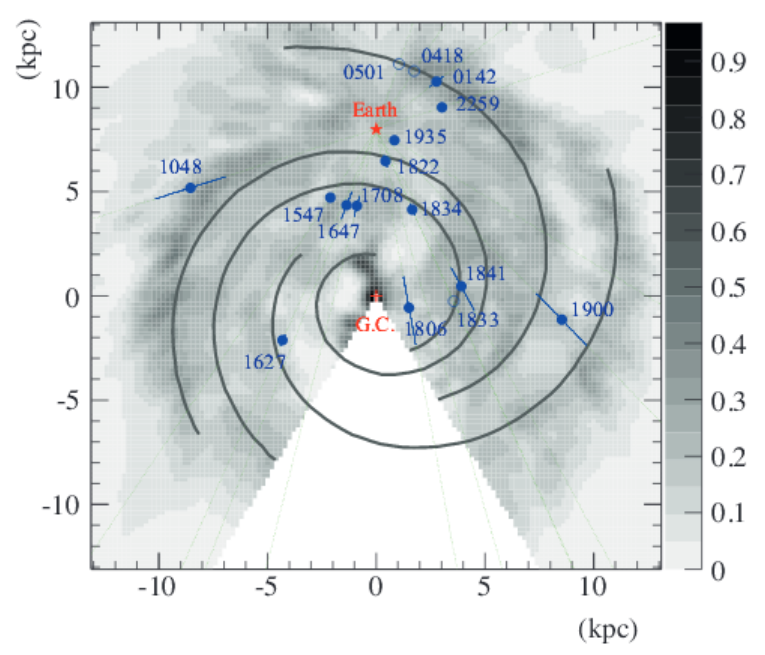

Figure 15: Up-to-date location of magnetars overlaid on the atomic hydrogen density (Nakanishi \& Sofue, 2003), from Enoto et al. (2017) (adopted from Enoto, 2018). 
up-to-date location of magnetars (Enoto, 2018).

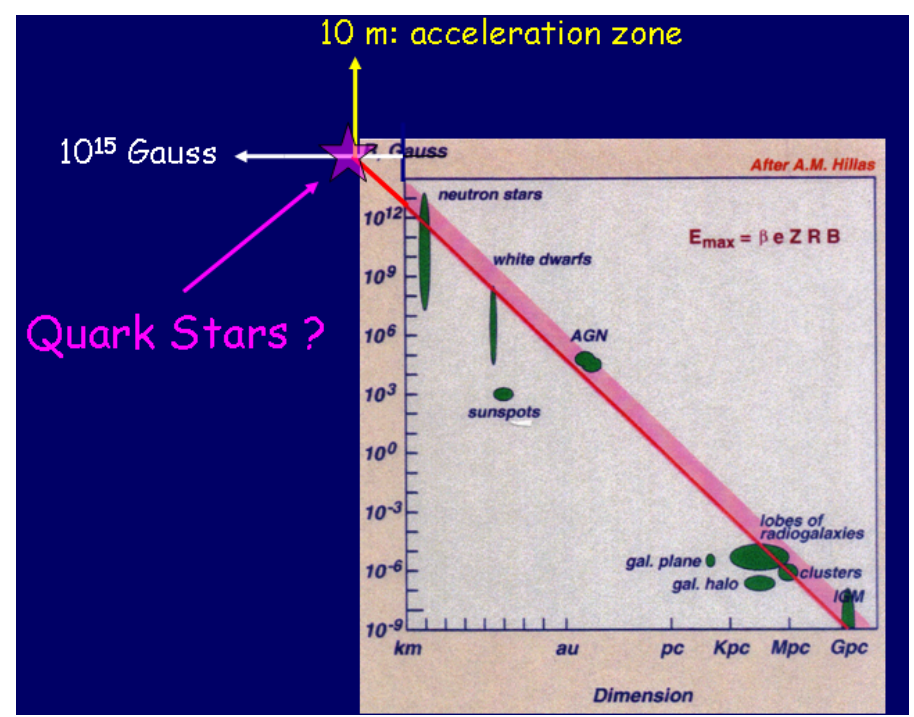

Figure 16: Magnetic field intensity vs dimension of cosmic sources (courtesy of Todor Stanev, 2002, after Hillas, 1984). The extrapolation to $10^{15} \mathrm{G}$ provides a dimension of $\sim 10 \mathrm{~m}$ : the acceleration zone in a supercompact star (quark star?) (adopted from Giovannelli \& Sabau-Graziati, 2006).

It is important to remind that a short history of the discovery of AXPs can be found in the review paper by Giovannelli \& Sabau-Graziati (2004), and references therein. Giovannelli \& SabauGraziati (2006) speculated as follows: "if magnetic fields of $\sim 10^{15}$ can be expected in order to explain the behaviour of magnetars, an almost 'obvious' consequence can be derived from the diagram magnetic field intensity versus the dimension of the relative cosmic source, like shown in Fig. 16 (courtesy of Todor Stanev, 2002), after Hillas, 1984). In this figure we have extrapolated the value of $B$ up to $10^{15} \mathrm{G}$. The corresponding dimension of the source is of $\sim 10 \mathrm{~m}$. This could be the dimension of the acceleration zone in a supercompact star, probably a "quark star". If you construct a trap, the rat falls into it!

\subsection{Neutrinos}

One of the most important questions of fundamental physics, that is still unanswered today, is the reason for our existence, namely why the Universe is made up mostly of matter. To put it in more microscopic terms, the important unanswered question relates to a theoretical understanding of the magnitude of the observed Baryon Asymmetry in the Universe (BAU). According to the Big Bang theory, matter and antimatter have been created at equal amounts in the early Universe. The observed charge-parity (CP) violation in particle physics (Christenson, et al., 1964), prompted Sakharov (1991a,b) to conjecture that non-equilibrium physics in the early Universe produces Baryon number (B), charge (C) and charge-parity (CP) violating, but CPT conserving, interactions/decays of anti-particles in the early Universe, resulting in the observed baryon-antibaryon asymmetry. In fact there are two types of non-equilibrium processes in the early Universe that could produce this asymmetry: the first type concerns processes generating asymmetries between leptons and anti-leptons (Leptogenesis), while the second produces asymmetries between baryons and anti-baryons (Baryogenesis) (Abazajian et al., 2012). 
The knowledge of the neutrino physics is fundamental for answering to this fundamental question. However, we cannot enter in a deep discussion about the physics of neutrinos. This argument deserves particular attention and space for discussion. A deep and exhaustive discussion about neutrinos can be found in the Light Sterile Neutrinos: A White Paper (Abazajian et al., 2012), Neutrino Oscillation Physics Potential of the T2K Experiment (Abe et al., 2015), and in Neutrino Physics with JUNO (An et al., 2015). However, we want to spend a few words about an important result obtained few years ago.

We know that neutrino oscillations are consistently described by three families $v_{1}, v_{2}, v_{3}$ with mass values $\mathrm{m}_{1}, \mathrm{~m}_{2}$ and $\mathrm{m}_{3}$ that are connected to the flavor eigenstates $v_{e}, v_{\mu}$ and $v_{\tau}$ by a mixing matrix U. The neutrino oscillation probability depends on: i) three mixing angles, $\Theta_{12}, \Theta_{23}, \Theta_{13}$; ii) two mass differences, $\Delta \mathrm{m}_{12}^{2}=\mathrm{m}_{22}-\mathrm{m}_{21}, \Delta \mathrm{m}_{23}^{2}=\mathrm{m}_{23}-\mathrm{m}_{22}$; iii) and a Charge-Parity (CP) phase $\delta_{\mathrm{CP}}$. The mixing angle $\Theta_{13}$ is the key parameter of three-neutrino oscillations and regulates at the first order all the oscillation processes that could contribute to the measurement of mass hierarchy and leptonic CP violation (Mezzetto, 2011). Indeed, the neutrino mixing angle $\Theta_{13}$ is at the focus of current neutrino research. Fogli et al. (2008) reported hints in favor of $\Theta_{13}>0$ at 90\% C.L.. Such hints are consistent with the recent indications of $v_{\mu} \rightarrow v_{e}$ appearance in the T2K (Abe et al., 2011) and MINOS long-baseline accelerator experiments as reported by Fogli et al. (2011). They found $\sin ^{2} \Theta_{13}=0.021 \pm 0.007$ or $\sin ^{2} \Theta_{13}=0.025 \pm 0.007$, depending on reactor neutrino flux systematics.

The evidence for $\sin ^{2} \Theta_{13}>0$ opens the door to CP violation searches in the neutrino sector, with profound implications for our understanding of the matter-antimatter asymmetry in the universe. Fogli et al. (2012) found interesting indications for $\Theta_{23}<\pi / 4$ and possible hints for $\delta \sim \pi$, with no significant difference between normal and inverted mass hierarchy.

Recent results coming from the OPERA experiment - designed to perform the most straightforward test of the phenomenon of neutrino oscillations (Acquafredda et al., 2009) - allowed to affirm that $v_{\mu} \rightarrow v_{\tau}$ oscillations really appeared in the CERN to Gran Sasso Neutrino beam (CNGS) with $6.1 \sigma$ significance obtained with the complete data sample, corresponding to $5603 v$ interactions fully reconstructed.

The values obtained are $\left|\Delta \mathrm{m}_{32}^{2}\right|=\left(2.7_{-0.6}^{+0.7}\right) \times 10^{-3} \mathrm{eV}^{2}$, assuming $\sin ^{2} 2 \Theta_{23}=1$ (Agafonova et al., OPERA Collaboration, 2018).

\section{Habitable Zone in the Milky Way and Exoplanets}

A deep discussion about this fundamental problem has been published by Giovannelli \& Sabau-Graziati (2016a) and references therein. We can briefly summarize in the following.

The most important questions about the possible origin of life in our Universe became a real scientific question in the last couple decades when it appeared a near certainty that other planets must orbit other stars. And yet, it could not be proven, until the early 1990's. Then, radio and optical astronomers detected small changes in stellar emission which revealed the presence of first a few, and now many, planetary systems around other stars. We call these planets "exoplanets" to distinguish them from our own solar system neighbors (http://science.nasa.gov/astrophysics/focusareas/exoplanet-exploration/). 
The research of potential habitable exoplanets has been strongly supported during last two decades. Indeed, this field of astrophysics is now probably the most exciting since the discovery of planets Earth-like could open a serious debate about the possibility of life outside of our solar system.

The presence of numerous exoplanets in the vicinity of our solar system - as detected by the Kepler observatory within a distance of $\approx 1 \mathrm{kpc}$ - plays an important role in speculating about the possible number of such exoplanets within the whole habitable zone (HZ) of our galaxy. Such HZ has an internal radius of $\sim 4 \mathrm{kpc}$ and an external radius of $\sim 11 \mathrm{kpc}$, as shown in Fig. 17, where the $\mathrm{HZ}$ in a Milky Way-like galaxy is represented in green. The number of stars contained in this zone is $\approx 10 \%$ of the total number of stars in the Galaxy. Taking into account that the thickness of the disk is $\approx 1 \mathrm{kpc}$, as evaluated by the differential rotation of the Galaxy, the habitable volume is $\sim 330 \mathrm{kpc}^{3}$.

The Kepler observatory confirmed the detection of $\gtrsim 4000$ exoplanets within $\approx 3.3 \mathrm{kpc}^{3}$, and 30 of them Earth-size (NASA Page Last Updated: April 13, 2018). Therefore, in the HZ of our galaxy we could expect $\approx 4 \times 10^{5}$ exoplanets, and 3000 of them Earth-size. This is the lower limit to the number of Earth-size exoplanets in the HZ of our galaxy. Indeed, planets around other stars are the rule rather than the exception, and there are likely hundreds of billions of exoplanets in the Milky Way alone (Maruyama, Ebisuzaki \& Kurokawa, 2018). Therefore, it is evident that the probability of finding numerous habitable planets becomes very high. Next generation instruments ground- and space-based will provide valuable information about this intriguing problem.

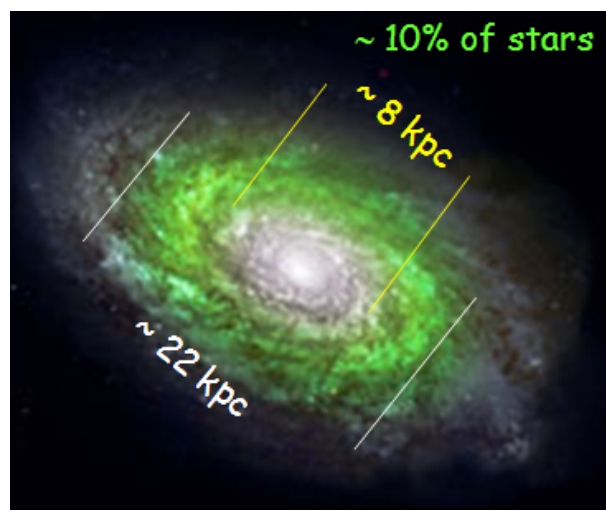

Figure 17: Habitable zone of a Milky Way-like galaxy (Giovannelli \& Sabau-Graziati, 2016a after Lineweaver, Fenner \& Gibson, 2004).

A strong support on the possibility of having numerous habitable planets is coming from the discovery of "usual" presence of water in the universe. We knew that all the water found on Earth, has been transported by small bodies such as comets and asteroids. On the contrary, the work "The ancient heritage of water ice in the solar system" (Cleeves et al., 2014) has carried the knowledge one step further. It is understood that the water now present in Earth's oceans, and is present in other solar system bodies, has remained virtually unchanged with respect to that in the interstellar medium. This means that this water has not changed during the process of planet formation. This allows us to understand that the initial conditions that have favored the emergence of life are not unique, i.e. not dependent on the unique characteristics of our solar system. They can, however, be 
common in space.

An intriguing question about the probability of finding a number of civilization in the Galaxy arises. It is now evident that Drake's formula (Drake, 1962) must be object of a robust revision.

For years, the search for manifestations of extraterrestrial civilizations has been one of humanity's most ambitious projects. Major efforts are now focused on the interception of messages from extraterrestrial civilizations, and the millimeter range is promising for these purposes (Dyson,1960). The Millimetron space observatory is aimed at conducting astronomical observations to probe a broad range of objects in the Universe in the wavelength range $20 \mu \mathrm{m}$ to $20 \mathrm{~mm}$, including the search for extraterrestrial life (Kardashev et al., 2014, and the references therein).

\section{Origin of terrestrial life}

The origin of life on Earth is one of the most intriguing fields of research because it stimulates the curiosity of the entire human race. To tackle such research, the skills of a multitude of scientists who are experts in the various fields of research are necessary: from geology, to paleontology, to biology, to chemistry, to physics, and to the new born astrobiology.

A multitude of articles and reviews have been published in the last twenty years. They discuss in depth the theme of the origin of life on Earth (e.g. Woese, 1998, 2000, 2002; Luisi, 2006; Arndt \& Nisbet, 2012; Koonin, 2014; Martin \& Sousa, 2016; Knoll, Bergmann \& Strauss, 2016 ; Kaiser, 2017; Cappellini et al., 2018; Martin, Bryant \& Beatty, 2018; de Vries \& Archibald, 2018).

It is our desire to venture into a short journey that gives the reader a chance to deepen the theme of the origin of life on the planet Earth and the further developments marked in a preponderant way by the presence of homo sapiens.

The Earth is 4.6 billion years old and microbial life is thought to have first appeared between 3.8 and 3.9 billion years ago; in fact, $80 \%$ of Earth's history was exclusively microbial life. Microbial life is still the dominant life form on Earth. It has been estimated that the total number of microbial cells on Earth on the order of $2.5 \times 10^{30}$ cells, making it the major fraction of biomass on the planet.

Phylogeny refers to the evolutionary relationships between organisms. The Three Domain System, proposed by Woese (1998), is an evolutionary model of phylogeny based on differences in the sequences of nucleotides in the cell's ribosomal RNAs (rRNA), as well as the cell's membrane lipid structure and its sensitivity to antibiotics. Comparing rRNA structure is especially useful. Because rRNA molecules throughout nature carry out the same function, their structure changes very little over time. Therefore similarities and dissimilarities in rRNA nucleotide sequences are a good indication of how related or unrelated different cells and organisms are.

There are various hypotheses as to the origin of prokaryotic and eukaryotic cells. Because all cells are similar in nature, it is generally thought that all cells came from a common ancestor cell termed the Last Universal Common Ancestor (LUCA). These LUCAs eventually evolved into three different cell types, each representing a domain. The three domains are the Archaea, the Bacteria, and the Eukarya (Kaiser, 2017).

Figure 18 shows the phylogenetic tree of life (Woose, 2000; Koonin, 2014; Kaiser, 2017).

The universal phylogenetic tree not only spans all extant life, but its root and earliest branchings represent stages in the evolutionary process before modern cell types had come into being. The 


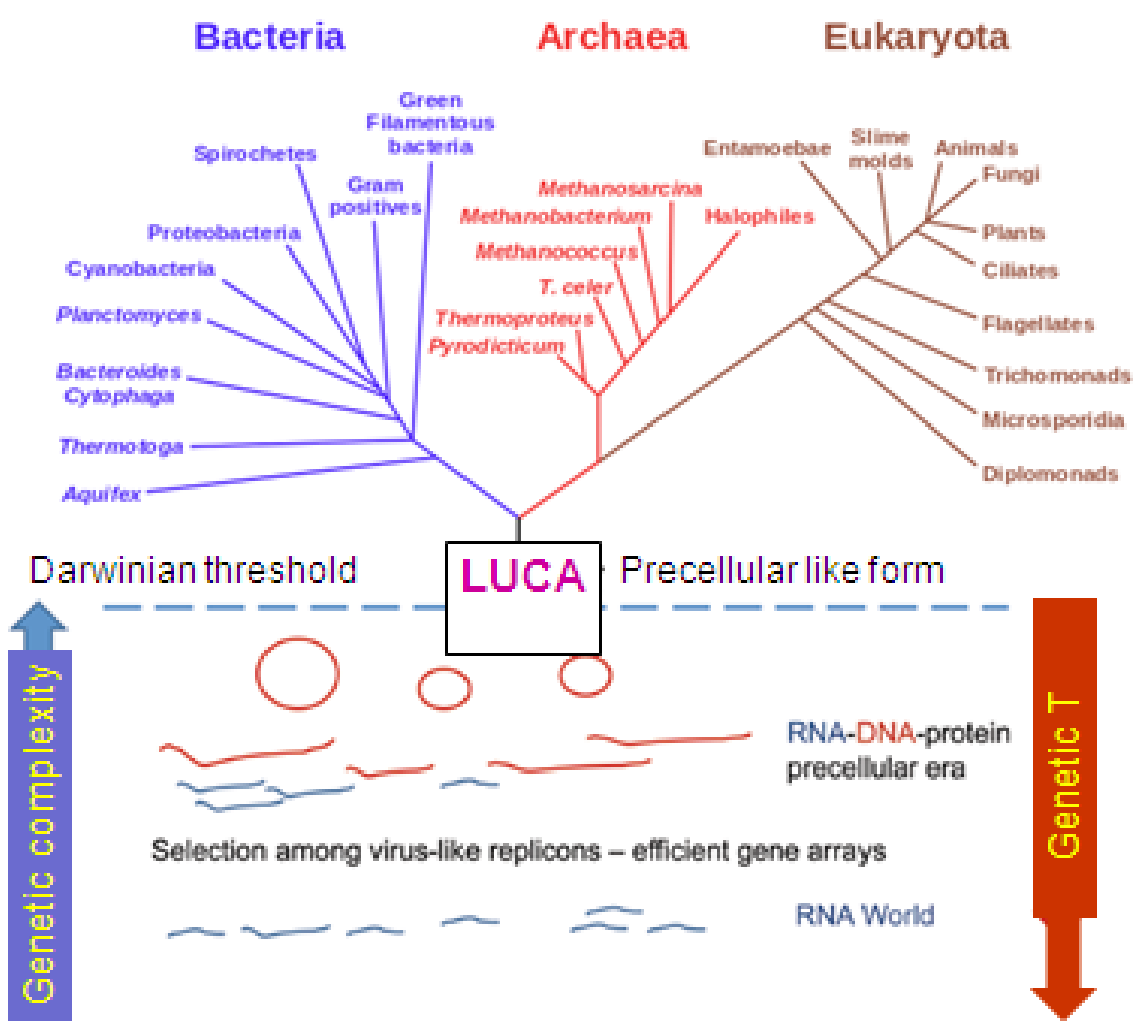

Figure 18: The phylogenetic tree of life (adapted from Kaiser, 2017, after Woese, 2000 and Koonin, 2014).

evolution of the cell is an interplay between vertically derived and horizontally acquired variation. Primitive cellular entities were necessarily simpler and more modular in design than are modern cells. Consequently, horizontal gene transfer early on was pervasive, dominating the evolutionary dynamic. The root of the universal phylogenetic tree represents the first stage in cellular evolution when the evolving cell became sufficiently integrated and stable to the erosive effects of horizontal gene transfer that true organismal lineages could exist (Woese, 2000).

Woese (2002) discussed a theory for the evolution of cellular organization. As a cell design becomes more complex and interconnected a critical point is reached where a more integrated cellular organization emerges, and vertically generated novelty can and does assume greater importance. This critical point is called the "Darwinian Threshold".

Phylogenomics and metagenomics have revealed enormous genetic and molecular diversity and extremely high abundance of viruses and related selfish genetic elements that come across as the dominant biological entities on earth. Furthermore, the perennial arms race between viruses and their cellular hosts is one of the defining factors of evolution. Microbial phylogenomics adds new dimensions to the fundamental picture of evolution, demonstrating that the traditional concepts based on the study of the evolution of multicellular animals and plants represent only the proverbial tip of the enormous iceberg of life's history. The scientist that made a greater contribution to this new era of evolutionary biology was Carl Woese (1928-2012) (Koonin, 2014).

Following the very interesting history of the "Early Life on Earth" (Four Peaks Technologies, 
2012a), we can take a short excursion on the evolution of life on our planet.

There is a general agreement that the first life on earth appeared sometime before $3.9 \mathrm{Gyr}$ ago. The origins of life are known to have come after the presence of liquid water on Earth. But other than that, there is no solid evidence to pin down a more precise date. However, once large pools of water had formed, it was possible for life to exist. There is carbon isotope evidence for life in the world's oldest known sedimentary rocks from the Isua Greenstone Belt of West Greenland estimated to be 3.85 Gyr old. These carbon rich rock layers probably accumulated as plankton bacteria on the surface, died and settled to the ocean floor. These early life forms were not only alive, but capable of photosynthesis, that is inhaling carbon dioxide and exhaling oxygen. The earliest life form was very simple. It was almost certainly cyanobacteria (mistakenly called blue-green algae at times). We know it existed $3.9 \mathrm{Gyr}$ at the latest because the first life also left behind the first fossils. Assuming it took about 100 million years for life to progress to the point of photosynthesis (which is pretty sophisticated), life began at least 4.0 billion years ago. Indeed, Bell \& Harrison (2013 and the references therein) found evidence that life likely existed on earth at least $4.1 \mathrm{Gyr}$ ago. The researchers, led by Bell, studied more than 10,000 zircons searching for carbon, the key component for life. Zircons are heavy, durable minerals originally formed from molten rocks in Western Australia. They capture and preserve their immediate environment, which means they serve as time capsules. The carbon contained in zircon has a characteristic signature, a specific ratio of ${ }^{12} \mathrm{C}$ to ${ }^{13} \mathrm{C}$, that indicates the presence of photo-synthetic life. They identified 656 zircons containing dark specks and closely analyzed 79 of them using a technique that shows the molecular and chemical structure of ancient micro-organisms in three dimensions. One of the 79 zircons contained graphite, which is pure carbon, in two locations. The graphite is older than the zircon containing it. They know the zircon is $4.1 \mathrm{Gyr}$ old based on its ratio of uranium to lead. They do not know how much older the graphite is (Bell et al., 2015).

Much life on earth belongs to the eukaryote family, all the way from blue-green algae to human beings. All multicellular organisms are eukaryotes, including animals, plants and fungi. On a numerical count basis, eukaryotes represent a tiny minority of all living things. Even in the human body, there are 10 times more microbe prokaryotes than human cells. It is believed that certain cyanobacteria evolved into blue-green algae eukaryotes about 2.5 Gyr ago much, much later than when cyanobacteria first appeared. The origin of the eukaryotic cell is considered a milestone in the evolution of life since they include all complex cells and almost all multicellular organisms. It was the development of the nucleus, which allowed highly complex forms of life to eventually evolve.

Stromatolites are the longest living form of life on the planet. They can be traced back at least 3.5 Gyr. Current marine stromatolites are only several thousand years old and can be found in waters in Western Australia and the Bahamas. Stromatolites are rock-like objects formed in shallow waters by living single celled micro-organisms, cyanobacteria, bound together in successive layers of carbonate sediment grains. Cyanobacteria come in population densities of over 3 billion organisms per square meter. Each cyanobacteria cell secretes a sticky film of mucus that traps local sediment grains. The sediment grains are bound together by the mucus and the cyanobacteria then grows over the grains. The bacteria are mobile and they photosynthesize, so they move towards light from the sun. Because the cyanobacteria need sunlight to photosynthesize, the stromatolites are generally found in water less than $200 \mathrm{~cm}$ deep where there is considerable sunlight. 
Their mobility also allows them to keep up with the growing sediment layers. The first records of stromatolites began about 3.5 Gyr ago. Their presence indicates that even at such an early age, advanced prokaryotes were present, indicating that life on earth could have began much earlier, maybe as early as 4.0 byr. Stromatolites peaked about $1.25 \mathrm{Gyr}$ and then began to decline. Today marine stromatolites can be found only in isolated areas like Shark Bay, Australia and the Bahamas. As an example of their decline, at Lake Clifton in Western Australia, scientists are witnessing algae (eukaryotes) out competing cyanobacteria, caused by an increase in nutrient levels in the water (Moore \& Burne, 1994; Couradeau et al., 2011).

The first multicelled organisms are believed to have been red algae, which appeared sometime between 1.4 and 1.2 Gyr ago. This was about two billion years after stromatolites first appeared. Thus, more than one-half the time life has been present on Earth, it was occupied by only single cell organisms.

Ancient micro-fossils of red algae were preserved and have been found on Somerset Island in northern arctic Canada. These fossils are as old as $1.2 \mathrm{Gyr}$. The first multicellular organisms had certain characteristics that have defined all complex life forms since. Red algae invented sex and reproduced sexually. The male red algae releases sperm into the water which floats nearby coming into contact with the female's reproductive organ and fertilization occurs. Upon contact, the barriers dissolve inside of the female's reproductive organs. The male nucleus divides and onehalf merges with the female nucleus. The female develops a large bulb which eventually buds off from the rest of the algae. This bulb is essentially a juvenile red algae which needs only time and nutrients to grow to an adult.

Sexual reproduction using egg and sperm cells is characteristic of multicelled organisms and first appeared in red algae. This development allowed much more complex life forms (including humans) to eventually evolve. So if you go back far enough, we all have red algae to thank for our existence.

\subsection{The Cambrian explosion}

In the book "The Cambrian Explosion: The Construction of Animal Biodiversity", Erwin \& Valentine (2013) describe the Cambrian Period, which records one of the most extraordinary transitions in the history of life. In this book the authors synthesize research from many fields to explain why there was such remarkable novelty of animal forms. This book is an integrative work of the highest quality, covering one of the most fascinating and transformative periods in life's history.

The Cambrian Period (541-485 Myr ago) witnessed a wild explosion of new life forms. Along with new burrowing lifestyles came hard body parts like shells and spines. Hard body parts allowed animals to more drastically engineer their environments, such as digging burrows. A shift also occurred towards more active animals, with defined heads and tails for directional movement to chase prey. Active feeding by well-armored animals like trilobites may have further disrupted the sea floor that the soft Ediacaran creatures had lived on.

Trilobites were the dominant species during this period. Trilobites are extinct arthropods, animals with a hard skin shell and jointed legs. Trilobites were distant relatives of modern lobsters and horseshoe crabs. Trilobites had three (tri-lobe) segmented, rather flat, top plated bodies. They could curl up into balls for protection in seas that were increasingly filled with predators. Trilobites were the first animals to develop eyes. Trilobites came in many varieties and sizes. Their length 
was $\approx 10-70 \mathrm{~cm}$. Trilobites proved to be among the most successful and enduring of all prehistoric animals. More than 17,000 species are known to have existed and they survived for approximately $300 \mathrm{Myr}$ and then perished. A dramatic lowering of sea levels at the time probably contributed to their demise.

A dominant animal of the Cambrian Period was the giant anomalocaris, which trapped its prey with two claw-tipped appendages lined with hooks in the front of its mouth. Anomalocaris, which means abnormal shrimp, had true compound eyes. For the time in which it lived, the anomalocaris was a gigantic creature reaching lengths of up to $\approx 200 \mathrm{~cm}$. Anomalocaris was a free-swimming animal that undulated through the water by flexing its body like a modern dolphin. They fed on trilobites and other arthropods, worms and mollusks. Anomalocaris was the largest and most fearsome predator of the Cambrian Period. Sponges also grew in the Cambrian seas. These animals belong to the phylum "porifera" because of all the tiny pores in their bodies. One species of sponge from this period had many branches that made it look like a tree. Another type of sponge looked like an ice cream cone without the ice cream. Many of the sponges became extinct when water temperatures dropped at the end of the Cambrian period. The Cambrian Period ended with a mass extinction. The leading theory is that a period of continental glaciation occurred when the climate of the Earth cooled at the end of the Cambrian. It was suggested that the cold conditions wiped out much of the warm water organisms because they were cold intolerant. Advancing glaciers would have lowered the temperature and the levels of the shallow seas where so many marine species lived. Changes in the temperature and also the reduction of the amount of oxygen in the water would have meant the end for many species that could not readily adapt. The loss of their habitat and the increased competition among the remaining displaced species led to the demise of many of them - a truly mass extinction.

The diversification of animals that occurred over a geologically short period (the Cambrian explosion) is commonly believed that was triggered by an increase of atmospheric oxygen. However, a causal relationship between the Cambrian explosion and increasing atmospheric oxygen lacks convincing evidence.

Indeed, Hammarlund, von Stedingk \& Påhlman (2018) challenge that view. They start with the fact that hypoxia $\left(<1-3 \% \mathrm{O}_{2}\right)$ maintains cellular immaturity (stemness), whereas adult stem cells continuously - and paradoxically - regenerate animal tissue in oxygenated settings. Novel insights from tumour biology illuminate how cell stemness nevertheless can be achieved through the action of oxygen-sensing transcription factors in oxygenated, regenerating tissue. They suggest that these hypoxia-inducible transcription factors provided animals with unprecedented control over cell stemness that allowed them to cope with fluctuating oxygen concentrations. Thus, a refinement of the cellular hypoxia-response machinery enabled cell stemness at oxic conditions and, then, animals to evolve into the oxic realm. This view on the onset of animal diversification is consistent with geological evidence and provides a new perspective on the challenges and evolution of multicellular life.

\subsection{Plant life}

About 450 Myr ago, soon after the Cambrian Period, plants began to make their way onto land. The first plants needed a source of water for photosynthesis, so they were found on marsh land where they could easily obtain water from the damp soil. Because they did not have any tissue 
that conducted water very well, they had to stay close to a supply in order to obtain the water they needed for photosynthesis. One of the major steps in plant evolution was the widespread evolution of spores as a form of plant reproduction. Spores are unicell organisms that are mobile and can reproduce forming new plants. Because spores could migrate by wind from place to place, they allowed plants to spread across the land. Spores eventually evolved into seeds, which are the multicell reproduction organisms of most of our current day plants. Another major development about $430 \mathrm{Myr}$ was the first appearance of vascular systems within plants. These are plant veins that circulate water, chemicals, and minerals within the plant. About $375 \mathrm{Myr}$, plants that had root systems and leaves appeared for the first time. These advancements allowed plants of this era to become much larger and to function internally like plants of today. As more time passed, about 300 Myr, conifers appeared and thrived. Some of the trees of this family are pines, cedars, cypress, and massive redwoods. Conifers are cone bearing seed plants, mostly trees. The conifer family rapidly spread until massive conifer forests covered most of the planet. Ferns were also quite abundant as they grew well in the undergrowth of the large conifer forests (Four Peaks Technologies, 2012a).

\subsection{Animal life}

For at least 1.4 Gyr after the beginning of life, no animal ever treaded on land. One reason was it takes a long time for creatures to evolve from one species to another. Going from living in water to living on land was a major step and would have taken a major amount of time. Another reason might have been ultraviolet rays. For a long time, the Earth did not have an ozone layer. Any creature that ventured on to land for any length of time would have been destroyed by the deadly radiation. After an oxygenated atmosphere developed, an ozone layer formed and land was safer to tread. However, the first large animals to walk the Earth were probably walking fish who still lived in water. Initially there was no food on the land so there was no pressing reason for them to live there permanently.

Trace fossils are the evidence of life preserved in sediments as a result of the living activities of organisms. They include surface tracks, trails, subsurface burrows, as well as fecal material and the marks produced by dying animals. They are evidence left behind by living things, but not direct evidence of the creatures themselves. There is fossil evidence of animal tracks on land from about 530 Myr. These tracks were probably made by tiny arthropods, animals with no spinal column (invertebrates), but have an external skeleton, a segmented body, and jointed appendages. Arthropods include flies, insects, worms, crabs, scorpions, starfish and octopus. The overwhelming majority of animal species are invertebrates. Only about $4 \%$ of all animal species have a spinal column.

It is believed that tetrapods, four limbed animals having a spinal column (vertebrates), walked on land about $400 \mathrm{Myr}$ according to fossil evidence. Tetrapods were aquatic creatures that lived in swamps and shallow ponds but ventured onto land occasionally, perhaps to mate or to hide from enemies (e.g. Clack, 2009). On land there were no enemies while in the sea there were plenty of them. Tetrapods probably also walked on the floors of their shallow swamps and ponds. Eventually tetrapods took up permanent residence on land and survived on small insects and small plants most likely plant mats related to the green algae family. Tetrapods include amphibians, reptiles, birds, dinosaurs and mammals. The development of the vertebrate structure paved the way for more advanced animals and eventually humans (e.g. the book edited by Barrett \& Milner, 2012, in 
which articles about studies on the earliest four-legged vertebrates, lizards, marine reptiles, turtles, dinosaurs, birds and mammals, ranging in age from just after the origin of tetrapods to the origins of modern bird families, with an emphasis on Palaeozoic and Mesozoic faunas are presented; George \& Blieck, 2011; and Pardo et al., 2017 and references therein).

Recently, Gess \& Ahlberg (2018) found that the first tetrapods of Africa lived within the Devonian Antarctic Circle 360 Myr ago. Therefore, fossils of four-legged vertebrates also evolved in polar regions, and not just in the tropics as previously believed. The evolution of tetrapods from fishes during the Devonian period was a key event in our distant ancestry. New-found fossils are coming from the latest Devonian Waterloo Farm locality near Grahamstown in the Eastern Cape, South Africa.

At this point of the evolution, when the vertebrates appeared, the way for more advanced animals and eventually humans was opened.

\subsection{The evolution of humans}

Following the very interesting history of the "Early Humans" (Four Peaks Technologies, 2012b), we can take a short excursion on the evolution of humans on our planet.

While hunting for fossils in the Afar Triangle in Ethiopia, in November 1974, paleo-anthropologist Donald Johanson and graduate student Tom Gray stumbled upon the partial remains of a previously unknown species of an ape-like hominid.

The mysterious skeleton (nicknamed Lucy) was eventually classified as a 3.2 Myr old "Australopithecus afarensis" - one of humankind's earliest ancestors. The headline grabbing find filled in crucial gaps in the human family tree.

Her large pelvic opening suggested she was female, and wear on her wisdom teeth hinted she was probably around 20 years old when she died. She would have appeared more ape-like than human, with long arms and a protruding belly. Unlike knuckle dragging apes, however, the structure of her bones showed that she yet walked erect (Lieberman, 2012), and her brain was tiny (Johanson \& Maitland, 1981).

A very interesting revolutionary discovery demonstrated that "Lucy" was not alone (HaileSelassie et al., 2012).

Indeed, until 1995, the fossil record suggested there was only one pre-human species at any given time before $3 \mathrm{Myr}$ ago. It was thought each species gave rise to another new species through time in a linear manner. But the discovery of Australopithecus bahrelghazali from Chad in 1995 and Kenyanthropus platyops from Kenya in 2001 challenged this idea. These two species were not widely accepted, and instead considered as geographic variants of Lucy's species, Australopithecus afarensis.

The discovery of a 3.4 Myr old Burtele partial foot from the Woranso-Mille announced by Haile-Selassie in 2012 was the first conclusive evidence that another early human ancestor species lived alongside Australopithecus afarensis.

The Woranso-Mille paleontological study area in Ethiopia's Afar region reveals that there were at least two, if not three, early human species living at the same time and in close geographic proximity.

This key research site has yielded new and unexpected evidence indicating that there were multiple species with different locomotor and dietary adaptations. 
How did multiple closely related species manage to co-exist in a relatively small area? How did they partition the available resources? These new discoveries keep expanding our knowledge and, at the same time, raise more questions about human origins.

The fourth species was identified as Australopithecus deyiremeda, a 3.5 to 3.3 Myr old human ancestor species from Ethiopia (Haile-Selassie et al., 2015).

Beall (2016) reports in his article a summary of the evolution of the primates:

- 55 Myr ago - First primitive primates evolve;

- $15 \mathrm{Myr}$ ago - Hominidae (great apes) evolve from the ancestors of the gibbon;

- 8 Myr ago - First gorillas evolve. Later, chimp and human lineages diverge;

- 5.5 Myr ago - Ardipithecus, early "proto-human" shares traits with chimps and gorillas;

- 4 Myr ago - Ape like early humans, the Australopithecines appeared. They had brains no larger than a chimpanzee's but other more human like features;

- 3.9-2.9 Myr ago - Australoipithecus afarensis lived in Africa;

- 2.7 Myr ago - Paranthropus, lived in woods and had massive jaws for chewing;

- $2.3 \mathrm{Myr}$ ago - Homo habilis first thought to have appeared in Africa

- 1.85 Myr ago - First "modern" hand emerges;

- 1.8 Myr ago - Homo ergaster begins to appear in fossil record;

- 1.6 Myr ago - Hand axes become the first major technological innovation;

- $0.8 \mathrm{Myr}$ ago - Early humans control fire and create hearths. Brain size increases rapidly;

- 0.4 Myr ago - Neanderthals first begin to appear and spread across Europe and Asia;

- $0.2 \mathrm{Myr}$ ago - Homo sapiens - modern humans - appear in Africa;

- 0.04 Myr ago - Modern humans reach Europe.

Figure 19 shows a sketch of the human evolution (Four Peaks Technologies, 2012b).

A recent paper by Du et al. (2018) demonstrates that pattern and process in hominin brain size evolution are scale-dependent. Their findings provide a quantitative basis for developing and testing scale-explicit hypotheses about the factors that led brain size to increase during hominin evolution.

Cappellini et al. (2018) in their review conclude that studies of ancient biomolecules have come a long way-from retrieval of short sequences of mitochondrial DNA from late Holocene materials to the assembly of full nuclear genome sequences and characterization of proteins and lipids dating back millions of years. These studies have profoundly deepened our understanding of the origin of early life forms, adaptation and extinction processes, and past migrations and admixtures that gave rise to present-day biological diversity, including in our own species. Today, ancient biomolecules can provide direct insights into both the deep and recent evolutionary past, at a scale and level of detail that few would have predicted less than a decade ago. 


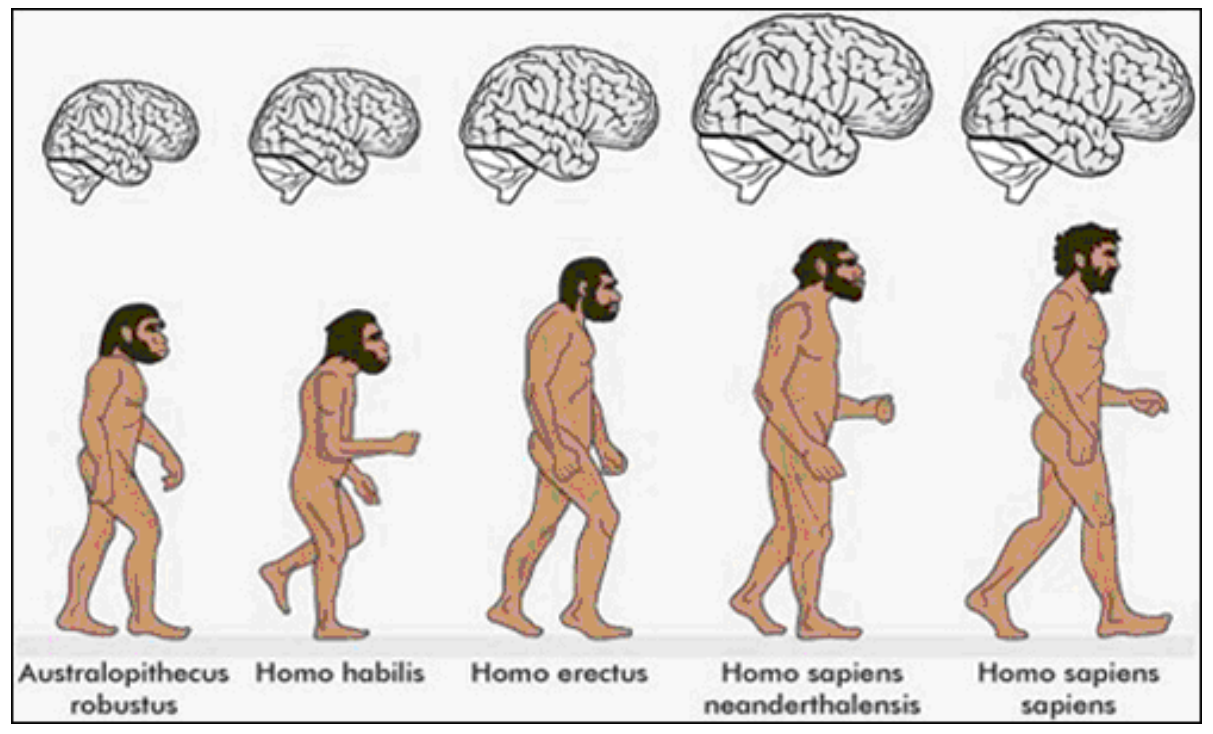

Figure 19: The complex evolution of humans (adopted from Four Peaks Technologies, 2012b).

\subsection{What intelligent humanity is doing?}

A short but important paper about The Future of Human Longevity: A Demographer's Perspective appeared in Science many years ago (Wilmoth, 1998). One of the greatest achievements of modern civilization has been the enormous reduction in human mortality. Life expectancy at birth, among early humans, was likely about 20 to 30 years (Preston, 1995). By 1900, the average length of life in industrialized nations had doubled relative to this historical extreme. Around the year 2000, life expectancy at birth was around 80 years in Japan and a few other countries, and its rise continues unabated. In recent decades, the populations of developed countries have grown considerably older, because of increasing survival to older ages as well as smaller numbers of births. Consequently, both legislators and the general public have begun to consider society's role in the support of this ever-expanding elderly population. In this new demographic context, questions about the future of human longevity have acquired a special significance for public policy and fiscal planning. Wilmoth (1998) discussed such a problem that is now extremely important for the future of the humanity.

It is important to remind a talk made by Wilmoth (2009) about the Increase of Human Longevity: Past, Present and Future, where he clearly discussed the following items:

- Historical increase of longevity

- Age patterns of mortality

- Medical causes of death

- Social and historical causes

- Limits to the human life span?

- Future prospects 
An important book about Life Span: Evolutionary, Ecological, and Demographic Perspectives (Carey \& Tuljapurkar, 2003) is based on a series of papers presented at the workshop held on 14-18 May 2001 at the Petros M. Nomikos Conference Centre on the Greek island of Santorini.

The volume is concerned with the biodemography of life span, a subject that has recently emerged at the confluence of demography and biology. Over the twentieth century, human life span measured as period life expectancy has increased by more than $50 \%$ in most industrialized countries, driving concern about the benefits and costs of longer life and raising keen interest in whether we can maintain the past rate of increase in life span. Biodemography brings together methods, materials, theories, and analyses from both biology and demography with the aim of understanding the past and anticipating the future of life span.

Without any doubt, life expectancy has grown considerably from prehistory to today. Figure 20 shows this evolution (Finch, 2012).

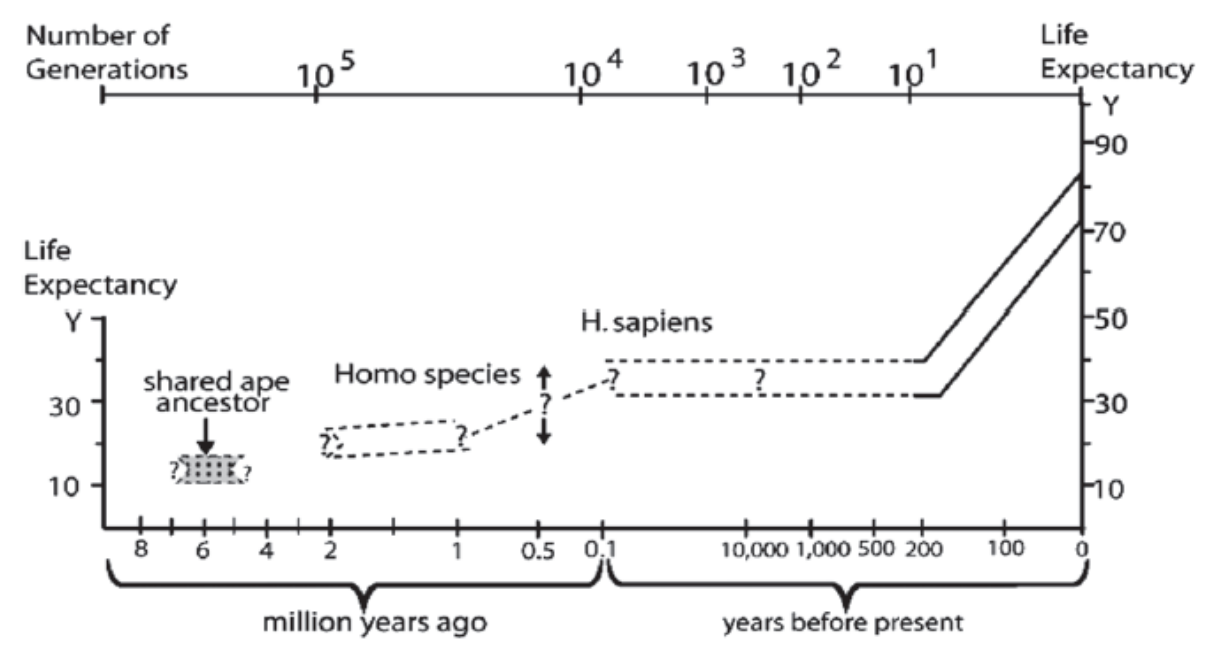

Figure 20: The evolution of the human life expectancy (Adopted from Finch, 2012).

However, the differences in the current world are at shameful levels. In fact, as shown in Figure 21, there is a direct correlation between the life expectancy at birth and the amount of energy available per capita (Pielke Jr., 2013).

The life expectancy at birth and the amount of energy available per capita is dramatically enforced by the effective average life of populations of different continents as shown in Fig. 22. The difference between Africa and Europe-North America-Japan are dramatically evident. Our responsibility for this state of affairs is evident and a great effort by the most fortunate countries is required to heal it.

A relationship between the life expectancy and the national income in current purchasingpower parity dollars shows how dramatic is the situation in the world about the "Income Inequality and Health, as pointed out by Deaton (2003).

Figure 23 shows a recent version of the Preston (1975) curve, the international relationship between life expectancy and national income in current purchasing-power parity dollars (after adjusting for inflation and cross-country price differences). Among the poorest countries, increases 


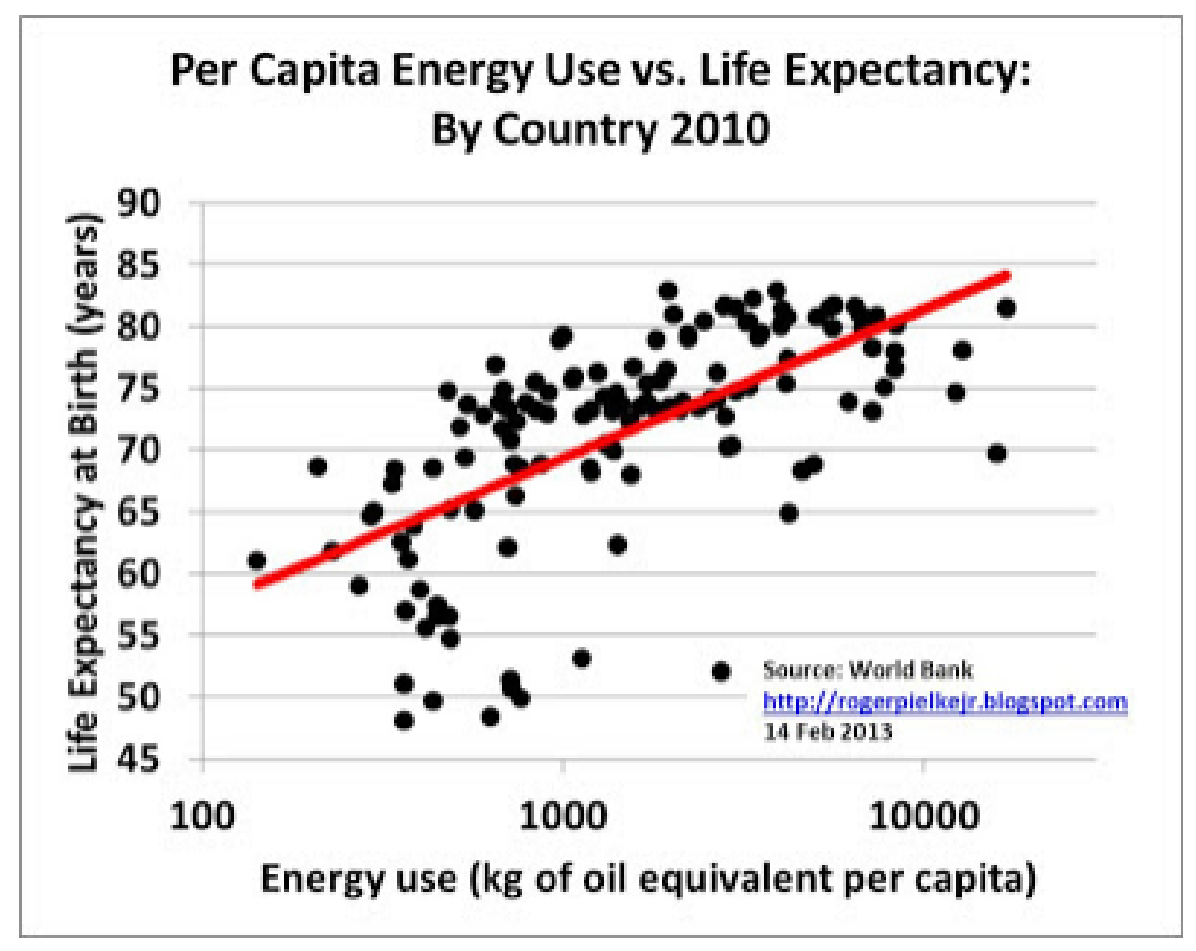

Figure 21: The energy use (expressed as kilograms of oil equivalent per capita) versus life expectancy at birth (expressed in years) for 151 countries in the "World Bank Development indicators database" that had data for both variables in 2010 (adopted from Pielke Jr., 2013).

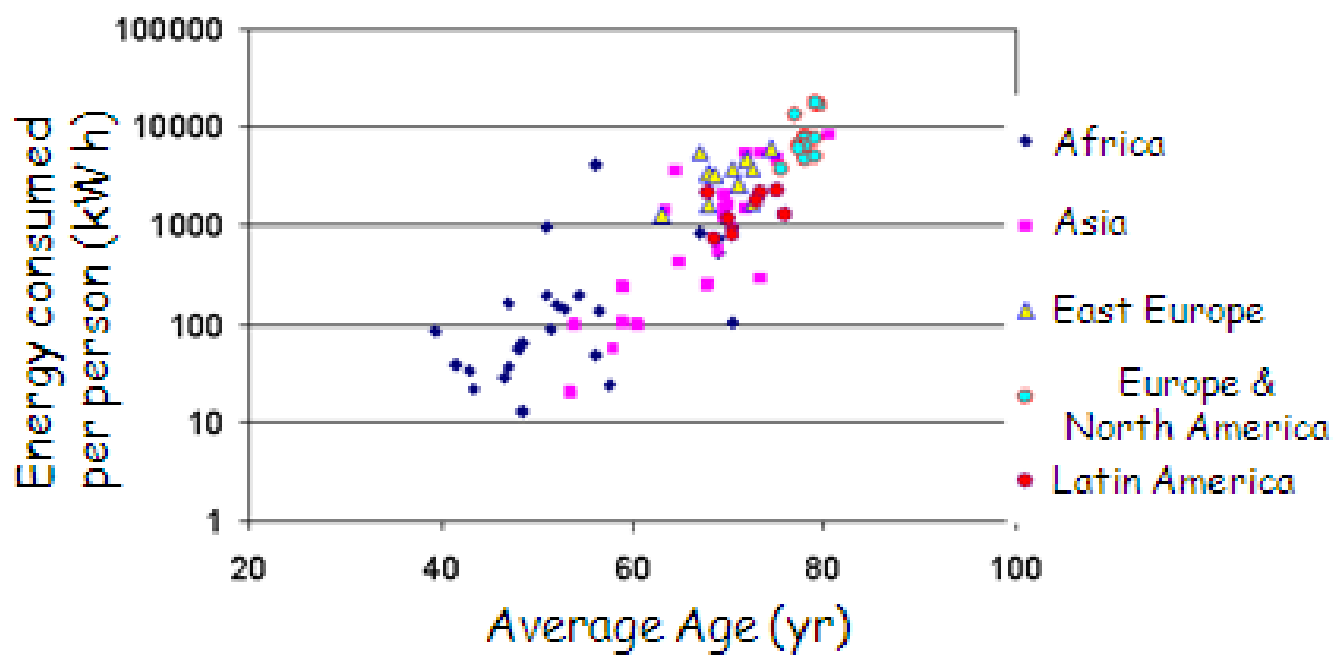

Figure 22: The average-life in years of populations belong to different continents versus the energy consumed pro capita in kWh (Adapted from Paolo Saraceno, 2009). 
in average income are strongly associated with increases in life expectancy, but as income per head rises, the relationship flattens out, and is weaker or even absent among the richest countries.

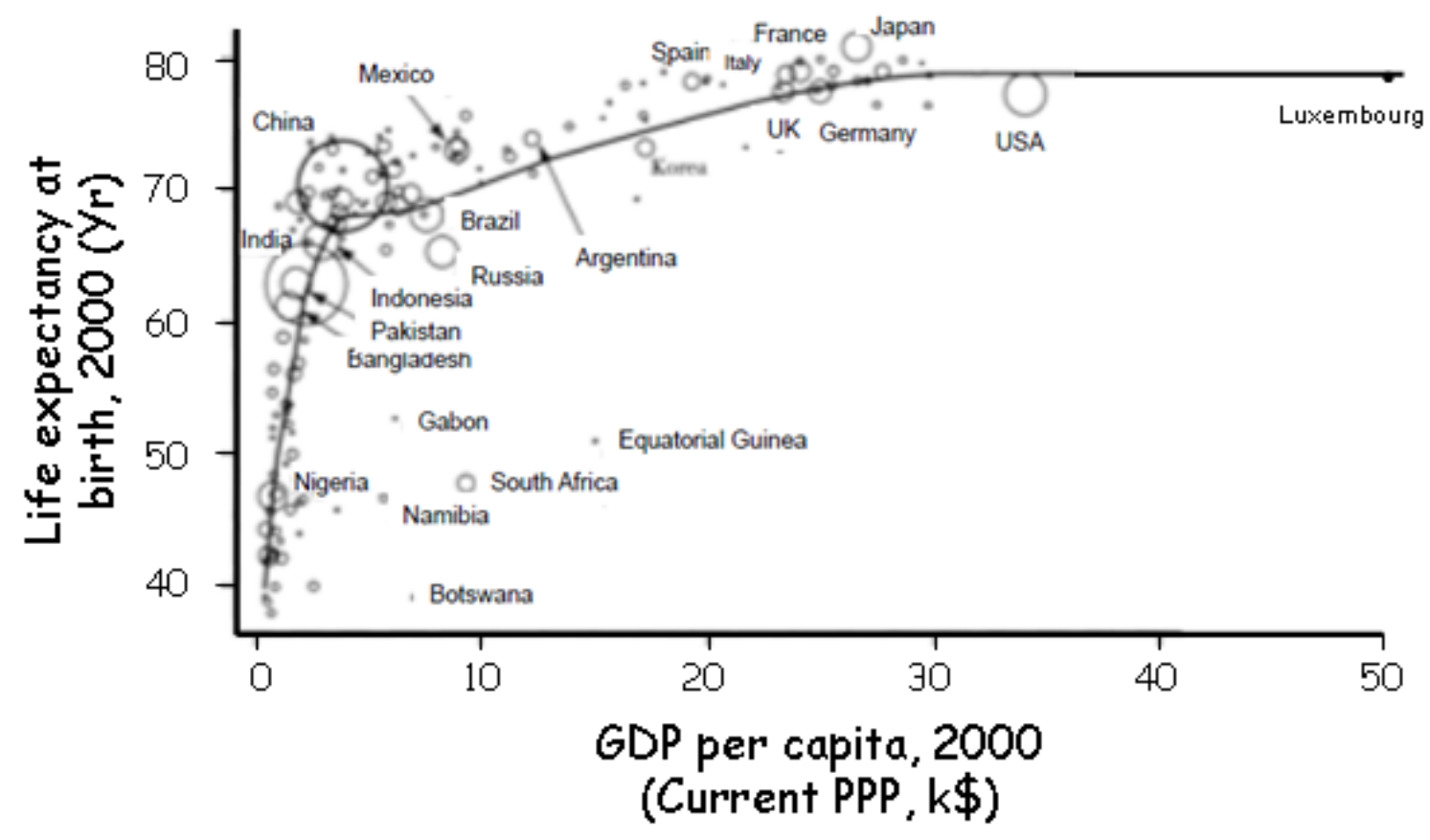

Figure 23: The Preston Curve updated: Life Expectancy versus Gross Domestic Product (GDP) per capita expressed in Current Purchasing-Power Parity (PPP) in kilo dollars) (updated from Deaton, 2003).

These data carry a powerful message: "Energy poverty is not the only factor which contributes to below the average life expectancies, but it is clearly a very important factor". Indeed, the countries having less energy available are the poorest ones and a relationship between the quality of life index (QL) - defined in a scale from 0 to 10 - versus the available energy per capita (ECR) (see Fig. 24: Pasten \& Santamarina, 2012) enforce the former conclusion.

Moreover, the quality of life index is strictly correlated to the five-year population growth rate $\mathrm{PG}_{5}$ (see Fig. 25: Pasten \& Santamarina, 2012). The poorer the countries the higher the birth rate is.

This increase in population closely correlated with the quality of life in different continents makes it possible to make fairly accurate predictions on the world population in the coming decades, as shown in Fig. 26 (United Nations, 2017).

Then, the crucial problem is associated with the overpopulation of the planet, which under optimal conditions could accommodate 2 billions people, as many as in 1950. The solution is anything but simple. In fact, it is not enough just to act well in everyday life. It is absolutely necessary to reduce the number of inhabitants or think seriously about colonizing the solar system.

An important review about the global population projections has been published by Lutz \& Samir (2010). These projections crossed with those reported in other many papers and in the world population previsions to 2300 reported by the United Nations (2004) allow to estimate that around 2075 the peoples of the Earth will have reached a good wellbeing and the population will begin to decrease because the children are: 


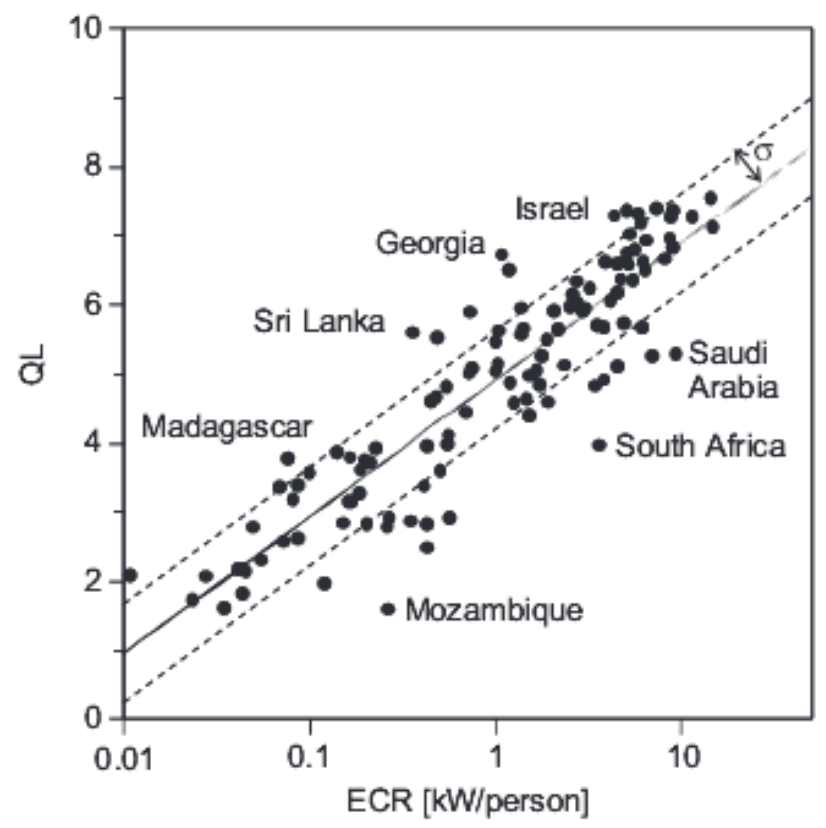

Figure 24: Quality of life index (QL) versus energy consumption rate per capita (ECR). Data for 118 countries with populations larger than four million in 2005. The continuous line is the mean trend; dashed lines show the plus and minus one standard deviation trends (adopted from Pasten \& Santamarina, 2012).

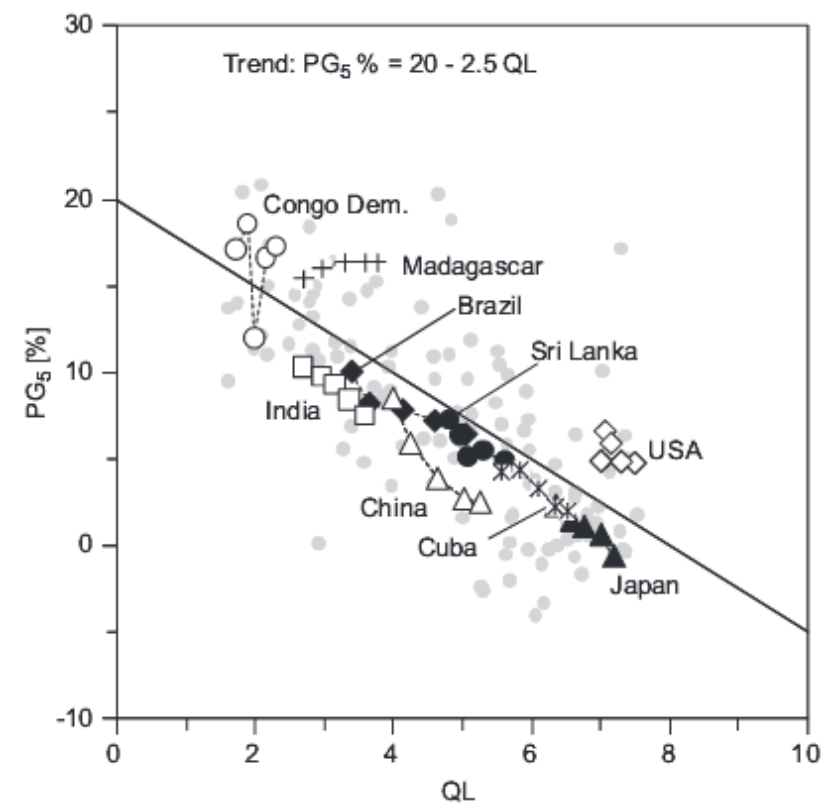

Figure 25: Five-year population growth rate $\mathrm{PG}_{5}$ and quality of life index $\mathrm{QL}$. The evolution of selected countries from 1980 to 2010 is shown in five-year intervals. The corresponding QL is the one at the beginning of the period. Dots represent 119 countries with populations larger than four million in the period 20052010. The line captures the global trend in the 30-year period from 1980 to 2010 (adopted from Pasten \& Santamarina, 2012). 
TABLE 1. Population OF THE WORLD AND REgIONS, 2017, 2030, 2050 AND 2100, ACCORDNG TO THE MEDIUM-VARIANT PROJECTION

\begin{tabular}{|c|c|c|c|c|}
\hline \multirow[b]{2}{*}{ Region } & \multicolumn{4}{|c|}{ Population (millions) } \\
\hline & 2017 & 2030 & 2050 & 2100 \\
\hline World .................. & 7550 & 8551 & 9772 & 11184 \\
\hline 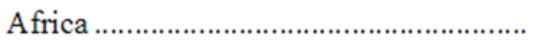 & 1256 & 1704 & 2528 & 4468 \\
\hline 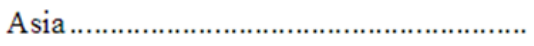 & 4504 & 4947 & 5257 & 4780 \\
\hline 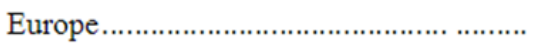 & 742 & 739 & 716 & 653 \\
\hline Latin America and the Caribbean .......... & 646 & 718 & 780 & 712 \\
\hline Northern America .............................. & 361 & 395 & 435 & 499 \\
\hline 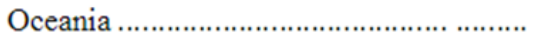 & 41 & 48 & 57 & 72 \\
\hline
\end{tabular}

Source: United Nations, Department of Economic and Social Affairs, Population Division (2017).

World Population Prospects: The 2017 Revision. New York: United Nations.

Figure 26: Population of the world and regions 2017, 2030, 2050, 2100 according to the medium-variant projection (United Nations, 2017).

- a wealth in poor and agricultural societies;

- a high cost in the rich and urbanized ones.

Figure 27 (upper panel) shows these estimates for the total world population, and for the populations belong to rich and poor countries, respectively (Saraceno, 2009). Figure 27 (lower panel) shows the historic reversal populations: the number of children between 0-14 years will become less than the number of people of age over 65 years (Chamie, 2016).

Estimates of the world population extended up to 2300 bring a wave of optimism to the future of humanity. In fact, in the hypothesis that humanity does not self-destruct, we could reach a decrease in the world population, for the benefit of the health of the planet, but only if we arrive at a more equitable distribution of wealth. This is the crucial point!

But unfortunately, the alarms of an imminent disaster are there for all to see. The temperature of the planet is increasing dramatically, as shown in Fig. 28 (Saraceno, 2012), and this can cause irreparable damage to the survival of the human species and beyond.

Indeed, for instance, the 2017 Hurricane Season Really Is More Intense Than Normal, like shown in Fig. 29. There have been 13 named storms in 2017. Only four other seasons since 1995 have had that many by Sept. 18. Just two more by the end of the year would put 2017 in the top 15 since 1851, when reliable records begin (Astor, 2017).

The winds of such hurricanes are extremely high, e.g.: Harvey $\left(\mathrm{v}_{\max }=215 \mathrm{~km} / \mathrm{h}\right), \operatorname{Irma}\left(\mathrm{v}_{\max }\right.$ $=285 \mathrm{~km} / \mathrm{h})$, Jose $\left(\mathrm{v}_{\max }=250 \mathrm{~km} / \mathrm{h}\right)$, Maria $\left(\mathrm{v}_{\max }=280 \mathrm{~km} / \mathrm{h}\right)$, and the tropical storm Katia $\left(\mathrm{v}_{\max }=\right.$ $120 \mathrm{~km} / \mathrm{h}$ ) (Wikipedia, 2017). It is superfluous to remind that the energy is proportional to $\mathrm{v}_{\max }^{2} ! ! !$ And the most important effects are evaluated in the provoked "damage" in U.S. dollars, without admitting that the effects of such hurricanes are son of the "stupidity" of humans!!!

In this regard, see the article by Giora Shaviv (2016). He discusses the phenomenon called Global Warming or in the new name Climate Change that refers to the behavior of the 'Temperature of the Earth' as a function of time. The 'temperature of the Earth' is a complicated term as it is measured in different locations and one has to define at what location and how to average the temperature over the complicated surface of the Earth so as to obtain a single number. The atmo- 

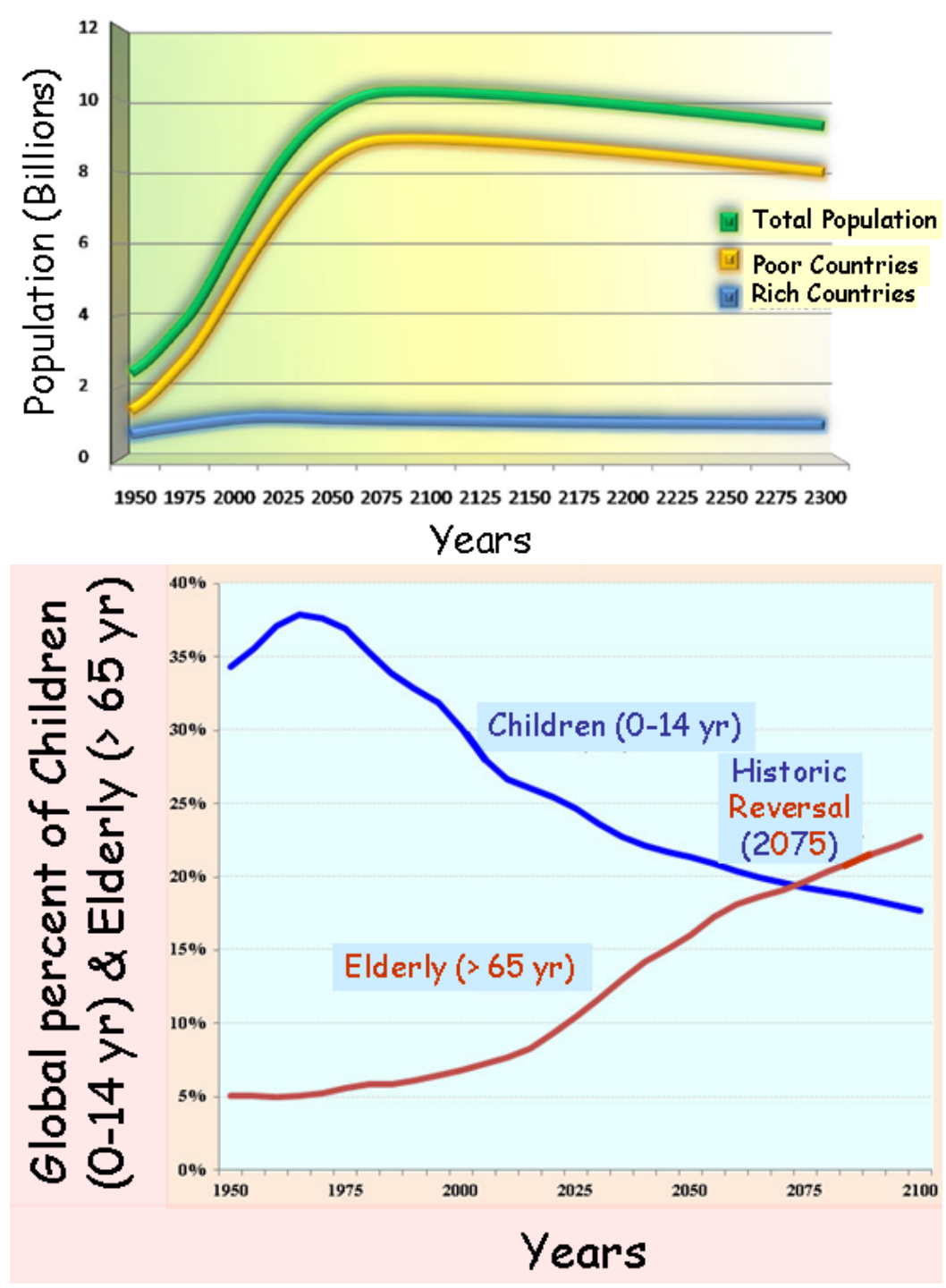

Figure 27: (Upper panel: World population estimate up to 2300 (adapted from Saraceno, 2009). Lower panel: Global percent of children (0-14 yr) and elderly (> $65 \mathrm{yr}$ ) from 1950 to 2100 (adapted from Chamie, 2016).

sphere, or better said the troposphere, can be considered as a boundary layer. However, this layer is not isothermal and shows large temporal and location temperature variations. The conclusions are not given because the problem is so complicated that the numerous theoretical models lead to significantly different results from each other.

The risk is that someone in the future will look at our species as we look at that of the dinosaurs!

Then, if we look for "Intelligent Lives" in the universe .... Why we should look for those like ours? (Giovannelli, 2001b). 

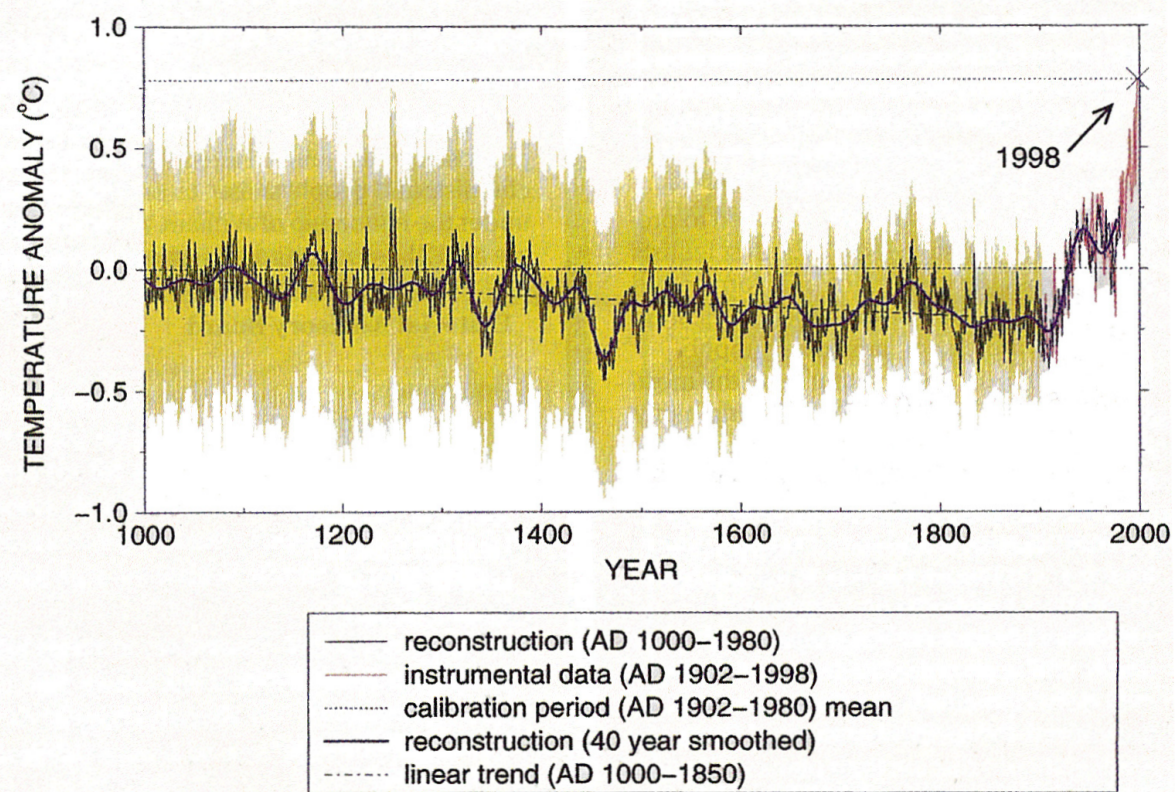

Figure 28: The temperature of the Earth from the year 1000 A.C. (adopted from Saraceno, 2012).

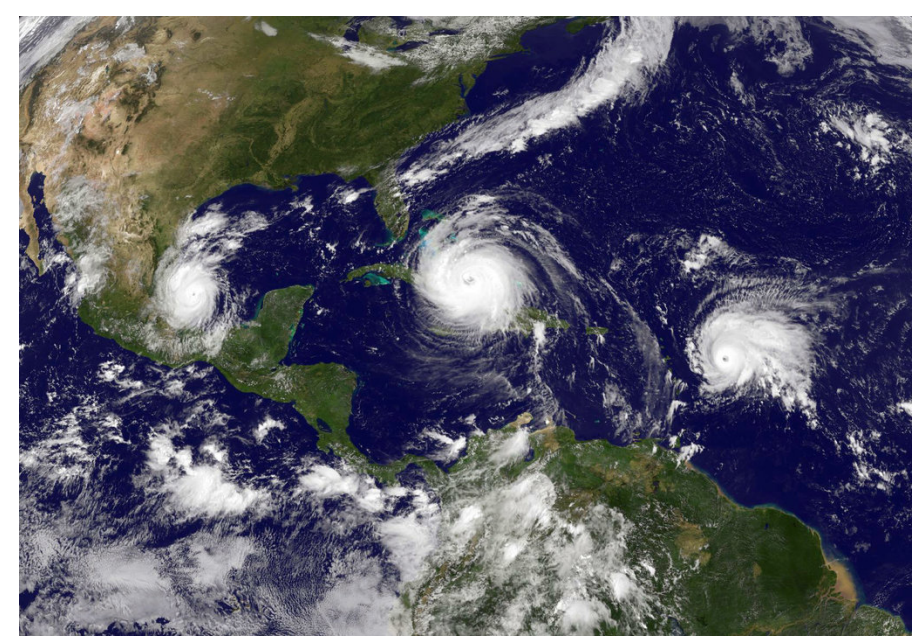

Figure 29: On Sept. 8, 2017 three storms threatened land: from left, Tropical Storm Katia, Hurricane Irma and Tropical Storm Jose, which would intensify into a hurricane (Adopted from Astor, 2017 - Credit: NOAA/NASA GOES Project). 


\section{Conclusions}

In this review paper we have discussed several arguments that in our opinion are fundamental for the comprehension of the physics of our Universe. We have emphasized with some examples the use of wisdom in physics.

It is important the Great example of synergy between Astrophysics and History we discussed for demonstrating that Sedov's formula for determining the age of SNRs can be revisited, thanks to the recalibration of the age of the SNR Vela Jr (Aschenbach, 2016) experimentally supported by a historical document.

We discussed about the small and big space- and ground-based experiments that provide measurements necessary for the advancement of the knowledge of the physics of our Universe. Thanks to these results we discussed the present situation about the problems resolved and those still open, far from the completeness due to our limited knowledge.

A section about the Habitable Zone in the Milky Way and Exoplanets briefly commented the fundamental problem about the research of life in the Universe. With this new-born field of science we could finally prove that the "Bridge between the Big Bang and Biology" (Giovannelli, 2001a) is, not only obviously existing, but really can be traveled over.

Indeed, we are going to study $\sim 50,000$ Clusters of Galaxies (Thomas Boller, 2017), and we have millions more. We know now more than 4000 planets clearly recognized in the Milky Way (Mullally, 2015). We know the lower limit of the extrapolated number of Earth-like planets in the habitable zone of our Galaxy that is $\approx 133 \times 10^{6}$. Thus we can dare to say that we are approaching to the philosophical results obtained by two great free thinkers:

- Siddhartha Gautama also known as Shakyamuni (the sage of Shaka - between the VI and V century B.C.) who exposes a grandiose vision of the universe: through the concept of "major system of worlds", a concept on huge scale that implies both the existence of countless galaxies and the possibility of sentient life on other planets other than our own (from the Lotus Sutra - the central text of Mahayana Buddhism).

- About 2000 years later, Giordano Bruno (Nola 1548 - Roma 17th February 1600) who was burned alive in Campo dei Fiori by the "Saint Inquisition" because of his thought - summarized in De l'infinito, universo e mondi (Giordano Bruno, 1584) (see Fig. 30)- that produced the same conclusions of Siddharta: The Universe is infinite and is populated by a myriad of worlds. Moreover he was saying that "Whether we like it or not, we are the cause of ourselves. Being born in this world, we fall into the illusion of the senses: we believe in what appears. We ignore that we are blind and deaf. Then the fear attacks us and we forget that we are "divine". We can change the course of events".

These philosophical lucubrations were not exactly in agreement with the position of the Roman (Catholic) Church!

Of course we must wait scientific confirmation for the "alien life". We must wait even more for the discovery of "intelligent life". But, the number of discovered planets is growing very fast. Thus, we can reasonably affirm that the Universe is full of life, hoping to avoid to be burned alive like Giordano Bruno. 


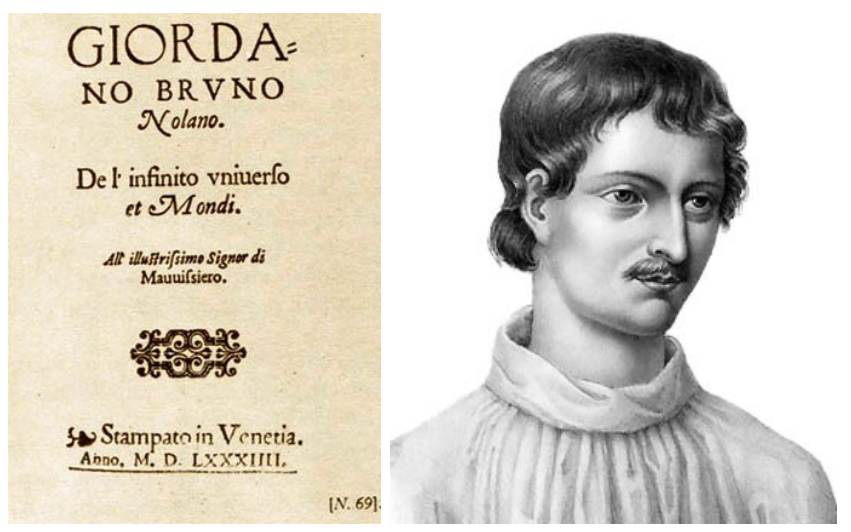

Figure 30: Left panel: Frontispiece of the original publication of De l'infinito, universo et mondi (Giordano Bruno, 1584). Right panel: Giordano Bruno (Rixner \& Siber, 1824).

In the final section Origin of terrestrial life and subsections we have discussed briefly the history of the emergence of life on the Earth, with particular emphasis about the behaviour of the humanity that for the first time in the history of the planet is able to provoke an artificial extinction.

The risk is that someone in the future will look at our species as we look at that of the dinosaurs!

Then, if we look for "Intelligent Lives" in the universe .... Why we should look for those like ours? (Giovannelli, 2001b).

As we discussed in the introduction, we can state that the Universe is interconnected in all its components: from cosmic network, to clusters of galaxies, to galaxies, to stars, to planets, to living beings, up to the simple bacterium. Therefore even every manifestation of life on our planet is subject to interconnection with all the surrounding environment. We can affirm that the whole Universe is a vital whole interconnected with more or less strong links between the various components, but that certainly exist.

Luisi and Capra (2014) discussed, in their excellent book, a new systemic conception of life, which shows an interesting convergence with our review paper. Indeed, new emphasis has been given to complexity, networks, and patterns of organization leading to a novel kind of 'systemic' thinking. This book integrates the ideas, models, and theories underlying the systems view of life into a single coherent framework. Taking a broad sweep through history and across scientific disciplines, the authors examine the appearance of key concepts such as autopoiesis (Maturana Romesín \& Varela García, 1972, 1980), dissipative structures, social networks, and a systemic understanding of evolution. The implications of the systems view of life for health care, management, and our global ecological and economic crises are also discussed. It is an essential reading for graduate students and researchers interested in understanding the new systemic conception of life and its implications for a broad range of professions - from economics and politics to medicine, psychology and law ... and physics.

Finally we can conclude with Fig. 31 that clearly explain all the mysteries of our Universe (Giovannelli, 2000). People who are able to read this sentence can understand that "The truth is written in the book of the Nature. We must learn to read this book".

The experiments provide the basic alphabet, immersed in an apparently chaotic soup, but 


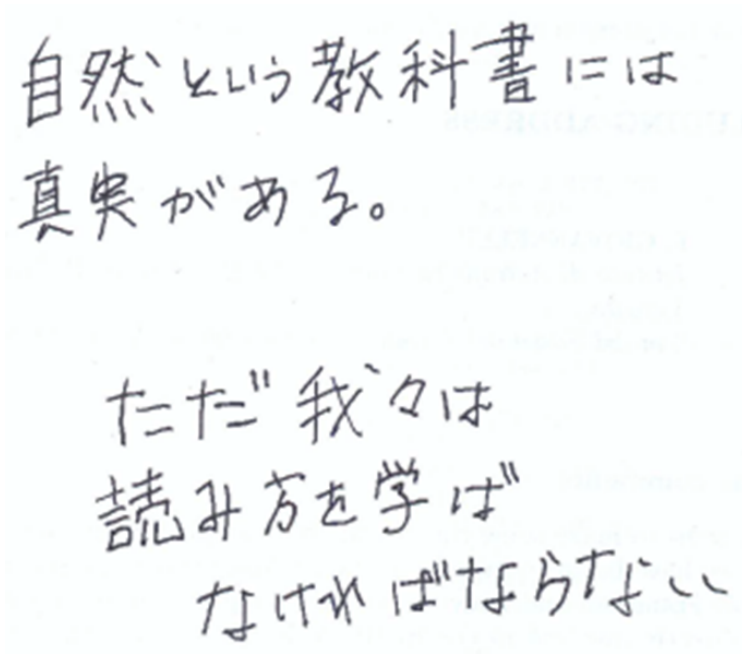

Figure 31: Understanding our Universe (Giovannelli, 2000).

necessary to understand the nature. From that soup we must extract words and phrases to compose the book of the nature. In other words, the data coming from the experiments constitute the basic alphabet that we use for constructing models that attempt to describe the nature. But we have a lot of models for interpreting the experimental data by the light of science. Depending on the hypotheses the results could run against the experiments. Then, in order to be acceptable, models can take into account and justify ALL the available data.

The same concept was expressed in much more incisive terms by Richard Phillips Feynman - Nobel laureate in Physics in 1965 - also known as The Great Explainer: It doesn't matter how beautiful your theory is, it doesn't matter how smart you are. If it doesn't agree with experiment, it's wrong.

\section{Acknowledgments}

This research has made use of "The NASA's Astrophysics Data System".

\section{References}

[1] Aad, G. (The ATLAS Collaboration): 2012, PhL B 718, 369-390.

[2] Aaij, R. et al.: 2015, arXiv:1507.03414v2 [hep-ex] 25 Jul.

[3] Abazajian, K.N. et al.: 2012, arXiv:1204.5379v1 [hep-ph] 18 Apr 2012

[4] Abbott, B.P. et al.: 2016a, PhRvL 116, 061102.

[5] Abbott, B.P. et al.: 2016b, PhRvL 116,1103.

[6] Abbott, B.P. et al.: 2016c, Living Rev. Relativity 19, 1.

[7] Abbott, B.P. et al.: 2017a, PhRvL 119, Issue 16, id.161101.

[8] Abbott, B.P. et al.: 2017b, ApJL 848, Issue 2, article id. L12, 59 pp. 
[9] Abdo, A.A., Ackermann, M., Ajello, M., Allafort, A., Atwood, W.B. et al.: 2010, ApJ, 723, Issue 2, 1082-1096.

[10] Abe, K., Abgrall, N., Aihara, H., Ajima, Y., Albert, J.B. et al.: 2011, Nucl. Instr. \& Meth. in Phys. Res. A, 659, Issue 1, 106-135.

[11] Abe, K., J. Adam, J., Aihara, H., Akiri, T., Andreopoulos, C. et al.: 2015, arXiv:1409.7469v2 [hep-ex] 10 Feb 2015.

[12] Acquafredda, R., Adam, T., Agafonova, N., Alvarez Sanchez, P., Ambrosio, M. et al.: 2009, J. Instr. 04, Issue 04, 04018.

[13] Ade, P.A.R. et al. (BICEP Collaboration): 2015, PhRvL 114, 1301.

[14] Adelberger, E.G., Austin, S.M., Bahcall, J.N., Balantekin, A.B., Bogaert, G. et al.: 1998, Rev. Mod. Phys., 70, 1265.

[15] Adelberger, E.G., García, A., Robertson, R.G. Hamish, Snover, K.A., Balantekin, A.B. et al.: 2011, Rev. Mod. Phys., 83, 195.

[16] Agafonova, N. et al., OPERA Collaboration: 2018, PhRvL 120, Issue 21, id. 211801.

[17] Amendola, L. et al. (The Euclid Theory Working Group): 2018, Living Reviews in Relativity, Volume 21, Issue 1, article id. 2, 345 pp.

[18] An, F., An, G., , An, Q., Antonelli, V., Baussan, E. et al.: 2015, arXiv:1507.05613v2 [physics.ins-det] 18 Oct 2015, 224 pp.

[19] Anders, M., Trezzi, D., Bellini, A., Aliotta, M., Bemmerer, D. et al.: 2013, EPJA, 49, id.28.

[20] Anders, M., Trezzi, D., Menegazzo, R., Aliotta, M., Bellini, A. et al. (LUNA Collaboration): 2014, Phys. Rev. Lett. 113, Issue 4, id.042501.

[21] Arndt, N.T., Nisbet, E.G.: 2012, Annu. Rev. Earth Planet. Sci. 40, 521-549.

[22] Aschenbach, B.: 1998, Nature 396, 141.

[23] Aschenbach, B.: 2016, in Frontier Research in Astrophysics II. Online at https://pos.sissa.it/cgi-bin/reader/conf.cgi?confid=269, id.36.

[24] Astor, M.: 2017, online at https://www.nytimes.com/2017/09/19/us/hurricanes-irma-harvey-maria.html

[25] Barkana, R.: 2018, Nature 555, Issue 7694, 71-74.

[26] Barkana, R., Loeb, A.: 2001, Phys. Rep. 349, 125.

[27] Barkana, R., Loeb, A.: 2005, ApJ 626, Issue 1, 1-11.

[28] Barrett, P.M, Milner, A.R. (Eds.): 2012, Special Papers in Palaeontology, Number 86, Studies on Fossil Tetrapods, Wiley-Blackwell, ISBN: 978-1-444-36189-6, 280 pp.

[29] Beall, A.: 2016, Article For Mailonline, Published: 15:03 BST, 7 June 2016 - Updated: 16:22 BST, 7 June 2016.

[30] Belczynski, K., Ziółkowski, J.: 2009, ApJ 707, 870.

[31] Bell, E.A., Harrison, T.M.: 2013, Earth and Planetary Science Letters, Vol. 364, 1-11.

[32] Bell, E.A., Boehnke, P., Hopkins-Wielicki, M.D., Harrison, T.M.: 2015, LITHOS, Vol. 234, 15-26. 
[33] Bennett, C.L., Larson, D., Weiland, J.L., Jarosik, N., Hinshaw, G.: 2013, ApJS 208, Issue 2, article id. 20,54 pp.

[34] Bennett, C.L., Larson, D., Weiland, J.L., Hinshaw, G.: 2014, ApJ 794, Issue 2, article id. 135, 8 pp.

[35] Berger, E., Fong, W. \& Chornock, R.: 2013, ApJL 774, Issue 2, article id. L23, 4 pp.

[36] Bernardini, M.G.: 2015, J.HE Astrophys. 7, 64-72.

[37] de Bernardis, P., Ade, P.A.R., Bock, J.J., Bond, J.R., Borrill, J. et al.: 2000, Nature 404, Issue 6781, 955-959.

[38] de Bernardis, P.: 2014, talk at the Mondello Workshop Frontier Research in Astrophysics - I.

[39] Bisnovatyi-Kogan, G.S.: 2002, in Black Hole Astrophysics 2002, Proc. of the Sixth APCTP Winter Schoo, Pohang, Korea, pp. 187-206.

[40] Bisnovatyi-Kogan, G.S., Giovannelli, F.: 2017, A\&A 599, id. A55, 7 pp.

[41] Blandford, R.D., Kochanek, C.S.: 2004, in Dark Matter in the Universe (Second Edition), J. Bahcall et al. (Eds.), World Scientific Publishing Co. Pte. Ltd., ISBN 9789812567185, pp. 103-158.

[42] Boller, T.: 2017, talk at the Frascati Workshop 2017 Multifrequency Behaviour of High Energy Cosmic Source - XII.

[43] Bowman, J.D., Rogers, A.E.E.: 2010, Nature 468, Issue 7325, 796-798.

[44] Bowman, J.D., Rogers, A.E.E., Monsalve, R.A., Mozdzen, T.J., Mahesh, N.: 2018, Nature 555, Issue 7694, 67-70.

[45] Broggini, C., Bemmerer, D., Guglielmetti, A., Menegazzo, R.: 2010, Annu. Rev. Nucl. Part. Sci. 60, 53-73.

[46] Broggini, C., Bemmerer, D., Caciolli, A., Trezzi, D.: 2018, Progr. in Part. and Nucl. Phys. 98, 55-84.

[47] Bruno, C.G., Scott, D.A., Aliotta, M., Formicola, A., Best, A. et al. (LUNA Collaboration): 2016, Phys. Rev. Lett. 117, Issue 14, id.142502.

[48] Bruno Giordano Nolano: 1584, De l'infinito, universo et mondi, Stampato in Venezia, Anno MDLXXXIV, in Dialoghi filosofici italiani, a cura di Michele Ciliberto, Mondadori, Milano (2000).

[49] Bucher, M. (on behalf of the PLANCK Collaboration): 2015, Nucl and Part. Phys. Proc. 267-269, 245-253.

[50] Buson, S. (Fermi-LAT Collaboration): 2014, in Frobtier Research in Astrophysics, Franco Giovannelli \& Lola Sabau-Graziati (Eds.), http://pos.sissa.it/cgi-bin/reader/conf.cgi?confid=237, id. 7.

[51] Cappellini, E., Prohaska, A. Racimo, F., Welker, F., Winther Pedersen, M. et al.: 2018, Annu. Rev. Biochem. 87, 1029-1060. Online at https://doi.org/10.1146/annurev-biochem-062917-012002

[52] Cardini, A.: 2015, Interview of Alessandro Cardini, P.I. of LHCb, 14th July 2015.

[53] Carey, James R., Tuljapurkar, Shripad (Eds.): 2003, Life Span: Evolutionary, Ecological, and Demographic Perspectives, Population and Development Review, A Supplement to Volume 29, 308 pp., Population Council, New York.

[54] Casares, J., Negueruela, I., Ribó, M., Ribas, I., Paredes, J.M. et al.: 2014, Nature 505, Issue 7483, 378-381.

[55] Cavanna, F., Depalo, R., Aliotta, M., Anders, M., Bemmerer, D. et al. (LUNA Collaboration): 2015, Phys. Rev. Lett. 115, Issue 25, id.252501. 
[56] Chamie, J.: 2016, Inter Press Service, http://www.ipsnews.net/2016/08/the-historic-reversal-of-populations/

[57] Chatrchyan, S. et al. (The CMS Collaboration): 2012, PhLB, 716, Issue 1, 30-61.

[58] Christenson, J.H., Cronin, J.W., Fitch, V.L., Turlay, R.: 1964, PhRvL, 13, Issue 4, 138-140.

[59] Clack, J.A: 2009, Evo Edu Outreach 2, 213-223.

[60] Class for Physics of the Royal Swedish Academy of Sciences: 2011, The accelerating Universe: Scientific Background on the Nobel Prize in Physics 2011.

[61] Cleeves, L. I., Bergin, E.A., Alexander, C.M.O.'D., Du, F., Graninger, D. Öberg, K.I. Harries, T.J: 2014, Science, 345, Issue 6204, 1590-1593.

[62] Cooray, A.: 2016, R. Soc. open sci. 3: 150555. http://dx.doi.org/10.1098/rsos.150555.

[63] Coppi, P.S., Aharonian, F.A.: 1997, ApJ, 487, Issue 1, L9-L12.

[64] Costamante, L.: 2012, in Multifrequency Behaviour of High Energy Cosmic Sources, F. Giovannelli \& L. Sabau-Graziati (Eds.), Mem. SAIt., 83, 138-145.

[65] Couradeau, E., Benzerara, K., Moreira, D., Gérard, E., Kaźmierczak, J., Tavera, R., López-García, P.: 2011, PLoS ONE, Vol. 6, Issue 12, e28767, pp. 1-16.

[66] Crites, A.T., Henning, J.W., Ade, P.A.R., Aird, K.A., Austermann, J.E. et al.: 2015, ApJ 805, 36, 18 pp.

[67] Cucchiara, A., Levan, A.J., Fox, D.B., Tanvir, N.R., Ukwatta, T.N. et al.: 2011, ApJ, 736, Issue 1, article id. 7, $12 \mathrm{pp}$.

[68] Dado, S., Dar, A., De Rújula, A.: 2009, ApJ 696, Issue 1, 994-1020.

[69] Dado, S., Dar, A.: 2013a, ApJ 775, Issue 1, article id. 16, 7 pp.

[70] Dado, S., Dar, A.: 2013b, A\&A 558, id.A115, 7 pp.

[71] Dado, S., Dar, A.: 2016, PhRvD 94, Issue 6, id.063007.

[72] Dai, X., Kochanek, C.S.: 2005, ApJ 625, Issue 2, 633-642.

[73] Dar, A.: 1997, in Very High Energy Phenomena in the Universe Morion Workshop. ISBN 2-86332-217-6 Editions Frontieres, Edited by Y. Giraud-Heraud and J. Tran Thanh Van, p.69. (arXiv:astro-ph/9704187v2).

[74] Dar, A.: 2017, talk at the Frascati Workshop 2017 Multifrequency Behaviour of High Energy Cosmic Sources - XIII.

[75] Dar, A., De Rújula, A.: 2000, arXiv:astro-ph/0008474.

[76] D'Avanzo, P.: 2015, J. HE Astrophys. 7, 73-80.

[77] Deaton, A.: 2003, Journal of Economic Literature, Vol. 41, No. 1, 113-158. Published online at https://www.jstor.org/stable/3217389

[78] Depalo, R., Cavanna, F., Aliotta, M., Anders, M., Bemmerer, D. et al. (LUNA Collaboration): 2016, Phys. Rev. C. 94, 055804.

[79] De Pasquale, M., Page, M.J., Kann, D.A., Oates, S.R., Schulze, S. et al.: 2016, MNRAS 462, Issue 1, 1111-1122. 
[80] Di Leva, A., Scott, D.A., Caciolli, A., Formicola, A., Strieder, F. et al. (LUNA Collaboration): 2014, Phys. Rev. C, 89, Issue 1, id.015803 and erratum Phys. Rev C, 90, Issue 1, id.019902.

[81] Drake, F.D.: 1962, Intelligent Life in Space, New York: Macmillan, 128 pp.

[82] Du, A., Zipkin, A.M., Hatala, K.G., Renner, E., Baker, J.L. et al.: 2018, in Hominin brain size evolution are scale-dependent, Proc. R. Soc. B 285: 20172738. http://dx.doi.org/10.1098/rspb.2017.2738

[83] Durrer, R.: 2015, Classical and Quantum Gravity, 32, Issue 12, article id. 124007.

[84] Dyson, F.: 1960, Science, 131, Issue 3414, 1667-1668.

[85] Enoto, T.: 2018, in Quarks and Compact Stars 2017 (QCS2017), JPS Conf. Proc., id.011046, 7 pp.

[86] Enoto, T., Shibata, S., Kitaguchi, T., Suwa, Y., Uchide, T. et al.: 2017, ApJ Suppl. Ser. 231, Issue 1, article id. 8, $21 \mathrm{pp}$.

[87] Erickson, J.K., Gratton, S., Steinhardt, P.J., Turok, N.: 2007, PhRvD 75, Issue 12, id. 123507.

[88] Erwin, D., Valentine, J.: 2013, The Cambrian Explosion: The Construction of Animal Biodiversity, Published January 18th by W.H. Freeman.

[89] Evans, P.A. et al.: 2009, MNRAS 397, 1177 [arXiv:0812.3662].

[90] Fabian, A.C. (Ed.): 1988, The DARWIN College lectures, Cambridge: University Press.

[91] Feng, C., Holder, G.: 2018, ApJL 858, L17, 4 pp.

[92] Finch, C,E,: 2012, Proc of the American Philosophical Soc. 156, 9-44.

[93] Flauger, R., Hill, J.C., Spergel, D.N.: 2014, JCAP 1408, 039. (arXiv:1405.7351).

[94] Fogli, G.L., Lisi, E., Marrone, A., Palazzo, A., Rotunno, A.M.: 2008, Ph. Rev. Letter, 101, Issue 14, id. 141801.

[95] Fogli, G.L., Lisi, E., Marrone, A., Palazzo, A., Rotunno, A.M.: 2011, Phys. Rev. D, 84, Issue 5, id. 053007.

[96] Fogli, G.L., Lisi, E., Marrone, A., Montanino, D., Palazzo, A., Rotunno, A.M: 2012, Phys. Rev. D, 86, Issue 1, id. 013012.

[97] Four Peaks Technologies, Scottsdale, AZ: 2012a, http://www.earlyearthcentral.com/early uderscore life underscore page.html

[98] Four Peaks Technologies, Scottsdale, AZ: 2012b, http://www.earlyearthcentral.com/human underscore page.html

[99] Fumagalli, M., Patel, S.G., Franx, M., Brammer, G., van Dokkum, P. et al.: 2012, APJL, 757, Issue 2, article id. L22, 6 pp.

[100] Gehrels, N., Cannizzo, J.K.: 2015, J. HE Astrophys. 7, 2-11.

[101] Gell-Mann, M.: 1964, PhL, 8, 214.

[102] George, D., Blieck, A.: 2011, PLoS ONE, Vol. 6, Issue 7, e22136, pp. 1-7.

[103] Gess, R., Ahlberg, P.E.: 2018, Science 360, 1120-1124.

[104] Ghirlanda, G., Bernardini, M.G., Calderone, G., D’Avanzo, P.: 2015, J. HE Astrophys. 7, 81-89.

[105] Giallongo, E., D’Odorico, S., Fontana, A., McMahon, R.G., Savaglio, S. et al.: 1994, ApJ, 425, no. 1, L1-L4. 
[106] Gianotti, F.: 2012, 4th July, talk at CERN.

[107] Giovannelli, F.: 2000, in The Evolution of The Milky Way, F. Matteucci \& F. Giovannelli (Eds.), Kluwer Academic Publishers, pp. 619-620.

[108] Giovannelli, F.: 2001a, The bridge between the big bang and biology: stars, planetary systems, atmospheres, volcanoes: their link to life, Franco Giovannelli (Ed.), Consiglio Nazionale delle Ricerche (CNR): President's Bureau of the CNR, Mario Apice (General Coordinator), pp. 1-440.

[109] Giovannelli, F.: 2001b, in The bridge between the big bang and biology: stars, planetary systems, atmospheres, volcanoes: their link to life, Franco Giovannelli (Ed.), Consiglio Nazionale delle Ricerche (CNR): President's Bureau of the CNR, Mario Apice (General Coordinator), p. 439.

[110] Giovannelli, F.: 2016, in High Energy Astrophysics in Southern Africa (HEASA 2016). Online at http://pos.sissa.it/cgi-bin/reader/conf.cgi?confid=275, id.31.

[111] Giovannelli, F., Sabau-Graziati, L.: 2004, SSR, 112, 1-443 (GSG2004).

[112] Giovannelli, F., Sabau-Graziati, L.: 2006, Chinese J. A\&A Suppl. 6, Issue S1, p. 1-28.

[113] Giovannelli, F., Sabau-Graziati, L.: 2011, Acta Polytechnica Vol. 51, No. 2., p. 21.

[114] Giovannelli, F., Sabau-Graziati, L. (Eds.): 2012, The Golden Age of Cataclysmic Variables and Related Objects - I, Mem. SAIt. 83, 433 pp.

[115] Giovannelli, F., Bisnovatyi-Kogan, G.S., Klepnev, A.S.: 2013, A\&A 560, id.A1, 11 pp (GBK13).

[116] Giovannelli, F., Bisnovatyi-Kogan, G.S., Bruni, I., Corfini, G., Martinelli, F., Rossi, C.: 2015, AcA $65,107-116$.

[117] Giovannelli, F., Sabau-Graziati, L.: 2014a, in Multifrequency Behaviour of High Energy Cosmic Sources - X, Acta Polytechnica CTU Proceedings 1(1), p.1-12.

[118] Giovannelli, F., Sabau-Graziati, L. (Eds.): 2015a, The Golden Age of Cataclysmic Variables and Related Objects - II, Acta Polytechnica, CTU Proc. Vol. 2, No. 1, 333 pp.

[119] Giovannelli, F., Sabau-Graziati, L. (Eds.): 2015b, The Golden Age of Cataclysmic Variables and Related Objects - III, Online at http://pos.sissa.it/cgi-bin/reader/conf.cgi?confid=255.

[120] Giovannelli, F., Sabau-Graziati, L.: 2016a, in Accretion Processes in Cosmic Sources, online at http://pos.sissa.it/cgi-bin/reader/conf.cgi?confid=288, id.1.

[121] Giovannelli, F., Sabau-Graziati, L. (Eds.): 2016b, Accretion Processes in Cosmic Sources: Young Stellar Objects, Cataclysmic Variables and Related Objects, X-ray Binary Systems, Active Galactic Nuclei, Online at http://pos.sissa.it/cgi-bin/reader/conf.cgi?confid=288.

[122] Giovannelli, F., Sabau-Graziati, L.: 2016c, in Frontier Research in Astrophysics II, Online at https://pos.sissa.it/cgi-bin/reader/conf.cgi?confid=269, id. 1 .

[123] Giovannelli, F., Sabau-Graziati, L.: 2017, in Multifrequency Behaviour of High Energy Cosmic Sources - XII, On line at https://pos.sissa.it/cgi-bin/reader/conf.cgi?confid=306, id. 1.

[124] Giovannelli, F., Sabau-Graziati, L. (Eds.): 2018a, The Golden Age of Cataclysmic Variables and Related Objects - IV, Online at http://pos.sissa.it/cgi-bin/reader/conf.cgi?confid=315.

[125] Giovannelli, F., Sabau-Graziati, L.: 2018b, in V Workshop on Robotic Autonomous Observatories, RevMexAA(SC) (in press).

[126] Gnedin, N.Y.: 2000, ApJ, 535, 530-554. 
[127] Greiner, J., Krühler, T., Fynbo, J.P.U., Rossi, A., Schwarz, R. et al.: 2009, ApJ, 693, Issue 2, 1610-1620.

[128] Gustavino, C.: 2007, in Frontier Objects in Astrophysics and Particle Physics, F. Giovannelli \& G. Mannocchi (Eds.), Italian Physical Society, Ed. Compositori, Bologna, Italy, 93, 191.

[129] Gustavino, C.: 2009, in Frontier Objects in Astrophysics and Particle Physics, F. Giovannelli \& G. Mannocchi (Eds.), Italian Physical Society, Ed. Compositori, Bologna, Italy, 98, 77.

[130] Gustavino, C.: 2011, in Frontier Objects in Astrophysics and Particle Physics, F. Giovannelli \& G. Mannocchi (Eds.), Italian Physical Society, Ed. Compositori, Bologna, Italy, 103, 657.

[131] Gustavino, C.: 2012, in Nuclei in the Cosmos (NIC XII), http://pos.sissa.it/cgi-bin/reader/conf.cgi?confid=146, id. 74

[132] Gustavino, C.: 2013, AcPol, 53, 534

[133] Haile-Selassie, Y., Saylor, B.Z., Deino, A., Levin, N.E., Alene, M., Latimer, B.M.: 2012, Nature 483, 565-600.

[134] Haile-Selassie, Y., Gibert, L., Melillo, S.M., Ryan, T.M., Alene, M. et al.: 2015, Nature 521, 483-498.

[135] Hammarlund, E.U., von Stedingk, K., Påhlman, S.: 2018, Nature Ecology \& Evolution Vol. 2, 220-228. DOI: 10.1038/s41559-017-0410-5.

[136] Harding, A.K.: 2013, Front. Phys., 8, Issue 6, 679-692.

[137] Haridasu, B.S., Lukovic, V.V., D’Agostino, R., Vittorio, N.: 2017, A\&A 600, id. L1, 5 pp.

[138] Hartwig, T., Volonteri, M., Bromm, V., Klessen, R.S., Barausse, E. et al.: 2016, MNRAS Lett. 460, Issue 1, L74-L78.

[139] Henry, R.C., Murthy, J., Overduin, J., Tyler, J.: 2015, ApJ, 798, Issue 1, article id. 14, 25 pp.

[140] Hillas, A.M.: 1984, ARA\&A 22, 425-444.

[141] Hogan, C.J., Rees, M.J.: 1979, MNRAS, 188, 791-798.

[142] Hopkins, A,M., Beacom, J.F., 2006, ApJ, 651, 142-154 - Erratum: 2008, ApJ 682, 1486.

[143] Ijjas, A.: 2018, Classical and Quantum Gravity Vol. 35, Issue 7, article id. 075010.

[144] Ijjas, A., Steinhardt, P.J., Loeb, A.: 2013, PhLB 723, 261.

[145] Ijjas, A., Steinhardt, P.J.: 2018, Classical and Quantum Gravity, Vol. 35, Issue 13, article id. 135004.

[146] Illarionov, A.F., Sunyaev, R.A.: 1975, A\&A 39, 185.

[147] Incandela, J.: 2012, UCSB/CERN, Talk on July 4, 2012.

[148] Izzo, L., Bernardini, M.G., Bianco, L.C., Caito, L., Patricelli, B., Ruffini, R.: 2010, J. Korean Phys. Soc., 57, issue 31, 551 .

[149] Jakobs, K., Seez, C.: 2015, Scholarpedia 10(9):32413.

[150] Johanson, D.. Maitland E.: 1981, Lucy: The Beginnings of Humankind, ISBN 0-671-25036-1, New York: Simon and Schuster.

[151] Kaiser, G,: 2017, in 1.3: Classification - The Three Domain System, https://bio.libretexts.org/.

[152] Kardashev, N.S., Novikov, I.D., Lukash, V.N., Pilipenko, S.V., Mikheeva, E.V., Bisikalo, D.V. et al.: 2014, Phys. Uspekhi, 57, Issue 12, article id. 1199-1228. 
[153] Kennicutt, R.C., Jr.: 1998, ARA\&A, 36, 189-232.

[154] Kennicutt, R.C., Evans, N.J.: 2012, ARA\&A, 50, 531-608.

[155] Knoll, A.H., Bergmann, K.D., Strauss, J.V.: 2016, Phil. Trans. R. Soc. B 371, 20150493. Online at http://dx.doi.org/10.1098/rstb.2015.0493

[156] Kochanek, C.S.: 2003, ASPC 291, 245.

[157] Komatsu, E., Smith, K.M., Dunkley, J., Bennett, C.L., Gold, B. et al.: 2011, ApJS, 192, Issue 2, article id. $18,47 \mathrm{pp}$.

[158] Koonin, E.V.: 2014, in Carl Woese special issue, www.landesbioscience.com, rNa Biology 11:3, 197-204.

[159] Kumar, P., Zhang, B.: 2015, PhR 561, 1-109.

[160] Lasota, J.-P.: 2001, New Astron. Rev. 45, 449-508.

[161] Lieberman, D.E.: 2012, Nature 483, 550-551.

[162] Lineweaver, C.H., Fenner, Y., Gibson, B.K.: 2004, Science 303, Issue 5654, 59-62.

[163] Lipunov, V.M.: 1987, Ap\&SS 132, no. 1, 1-51.

[164] Lipunov, V.M.: 1995, in Frontier Objects in Astrophysics and Particle Physics, F. Giovannelli \& G. Mannocchi (Eds.), SIF, Bologna, Italy, 47, 61-76.

[165] Lipunov, V.M., Postnov, K.A.: 1988, Ap\&SS 145, no. 1, 1-45.

[166] Luisi, P.L.: 2006, The Emergence of Life. From Chemical Origin to Synthetic Biology, Cambridge Univeristy Press.

[167] Luisi, P.L., Capra, F.: 2014, The systems View of Life, Cambridge Univ. Press, 510 pp.

[168] Lutz, W., Samir, K.C.: 2010, Phil. Trans. R. Soc. B 365, 2779-2791.

[169] Madau, P.: 2018, MNRAS 480, L43-L47.

[170] Madau, P., Dickinson, M.: 2014, ARA\&A, 52, 415-486.

[171] Magic Collaboration - Albert, J. et al.: 2008, Science, 320, 1752-1754.

[172] Martin, W.F., Sousa, F.L.: 2016, Cold Spring Harb Perspect Biol 8, a018127 - Online at http://cshperspectives.cshlp.org/

[173] Martin, W.F., Bryant, D.A., Beatty, J.T.: 2018, FEMS Microbiology Reviews, fux056, 42, 205-231. Online at http://creativecommons.org/licenses/by/4.0/

[174] Maruyama, S., Ebisuzaki, T., Kurokawa, K.: 2018, These proceedings.

[175] Maselli, A., Melandri, A., Nava, L., Mundell, C.G., Kawai, N. et al.: 2014, Science 343, Issue 6166, 48-51.

[176] Maturana Romesín, H., Varela García, F.J.: 1972, De Máquinas y Seres Vivos (Autopoiesis: La Organización de lo Vivo), Editorial Universitaria S.A., Chile.

[177] Maturana Romesín, H., Varela García, F.J.: 1980, Autopoiesis and Cognition - The Realization of the Living, D. Reidel Publishing Company Dordrecth, Holland.

[178] McKee, C.F., Ostriker, E.C.: 2007, ARA\&A, 45, Issue 1, 565-687. 
[179] Meylan, G., Jetzer, P., North, P., Schneider, P., Kochanek, C.S., Wambsganss, J.: 2006, Gravitational lensing: strong, weak and micro, G. Meylan, P. Jetzer \& P. North (Eds.), Berlin: Springer, ISBN 3-540-30309-X, ISBN 978-3-540-30309-1, XIII + 552 pp. (Kochanek, C.S.: 2004, arXiv:astro-ph/0407232).

[180] Mezzetto, M.: 2011, in Symposium on Prospects in the Physics of Discrete Symmetries, Journal of Physics: Conference Series 335, 012005.

[181] Moore, L.S., Burne, R.V.: 1994, in Phanerozoic Stromatolites II, J. Bertrand-Safati \& C. Monty (Eds.), Crown Copyright, Printed in the Netherlands, pp. 3-29.

[182] Mullally, F., Coughlin, J.L., Thompson, S.E., Rowe, J., Burke, C. et al.: 2015, ApJS, 217, Issue 2, article id. 31,16 pp.

[183] Nakanishi, H., Sofue, Y.: 2003, PASJ 55, 191-202.

[184] Nakar, E.: 2007, Phys. Rep. 442, 166-236.

[185] Nielsen, J.T., Guffanti, A., Sarkar, S. 2016, NatSR 6, id. 35596.

[186] Orosz, J.A., Remillard, R.A., Bailyn, C.D., McClintock, J.E.: 1997, ApJL 478, L83.

[187] Pardo, J.D., Szostakiwskyj, M., Ahlberg, P.E., Anderson, J.S.: 2017, Nature 546, 642-653.

[188] Pasten, C., Santamarina, J.C.: 2012, Energy Policy 49, 468-476.

[189] Perlmutter, S., Aldering, G., Goldhaber, G., Knop, R.A., Nugent, P. et al.: 1999, ApJ 517, Issue 2, 565-586.

[190] Pielke Jr., R.: 2013, at http://rogerpielkejr.blogspot.com/2013/02/graph-of-day-life-expectancy-vs-energy.html

[191] Piran, T.: 1999, Phys. Rep. 314, Issue 6, 575-667.

[192] Piron, F.: 2016, C. R. Physique 17, 617-631.

[193] Planck Collaboration: 2014, A\&A 571, id. A11, 37 pp.

[194] Preston, S.H.: 1975, Population Studies: A Journal of Demography, Vol. 29, Issue 2, 231-248. Published online 8th November 2011 at https://doi.org/10.1080/00324728.1975.10410201

[195] Preston, S.H.: 1995, in The State of Humanity, J. L. Simon (Ed.), Blackwell, Cambridge, pp. 30-36.

[196] Raguzova, N.V., Lipunov, V.M.: 1999, A\&A 349, 505.

[197] Rees, M.J.: 1988, in Origins, A.C. Fabian (ed.), Cambridge University Press, 1.

[198] Ressell, M.T., Turner, M.S.: 1990, Comm. Astrophys. 14, No. 6, 323-356.

[199] Riess, A.G., Filippenko, A.V., Challis, P., Clocchiatti, A., Diercks, A. et al.: 1998, AJ 116, Issue 3, 1009-1038.

[200] Riess, A.G., Strolger, L.-G., Tonry, J., Casertano, S., Ferguson, H.C. et al.: 2004, ApJ, 607, Issue 2, 665-687.

[201] Riess, A.G., Macri, L., Casertano, S., Lampeitl, H., Ferguson, H.C. et al.: 2011, ApJ, 730, Issue 2, article id. 119, 18 pp. - Erratum: 2011, ApJ, 732, 129.

[202] Rixner, T.A., Siber, T.: 1824, Leben und Lehrmeinungen berühmter Physiker, Sulzbach, Heft 5.

[203] Sakharov, A.D.: 1991a, Soviet Physics Uspekhi 34, Issue 5, 392-393. 
[204] Sakharov, A.D.: 1991b, Soviet Physics Uspekhi 34, Issue 5, 417-421

[205] Salvador Solé, E., Manrique, A, Canales, E.: 2018, These proceedings.

[206] Saraceno, P.: 2009, talk at Physics Dpt, La Sapienza University, Roma, Italy Energia e Ambiente: $i$ numeri che contano.

[207] Saraceno, P.: 2012, Beyond the Stars: Our Origins and the Search for Life in the Universe, World Scientific Publishing Co. Pte. Ltd.

[208] Scaringi, S.: 2015, talk at the Palermo Workshop on The Golden Age of CVs and Related Objects III.

[209] Schuecker, P.: 2005, In Rev. Mod. Astron. (Siegfried Röser Ed.), Vol. 18, 76-105.

[210] Schuecker, P., Caldwell, R.R., Böhringer, H., Collins, C.A., Guzzo, L., Weinberg, N.N.: 2003, A\&A, 402, 53-63.

[211] Scott, D.A., Caciolli, A., Di Leva, A., Formicola, A., Aliotta, M. et al.: 2012, Phys. Rev. Lett, 109, Issue 20, id. 202501.

[212] Shahbaz, T., Bandyopadhyay, R.M., Charles, P.A., Wagner, R.M., Muhli, P., et al.: 1998, MNRAS $300,1035$.

[213] Shandarin, S., Habib, S., Heitmann, K.: 2010, PhRv D, 81, Issue 10, id. 103006.

[214] Shakura, N.I., Sunyaev, R.A.: 1973, A\&A 24, 337.

[215] Shaviv, G.: 2016, in Accretion Processes in Cosmic Sources, Online at http://pos.sissa.it/cgi-bin/reader/conf.cgi?confid=288, id.60

[216] Shaviv, N.J., Dar, A.: 1995, MNRAS 277, Issue 1, 287-296.

[217] Smak, J.: 1984, PASP 96, 5-18.

[218] Smak, J.: 1985a, in Multifrequency Behaviour of Galactic Accreting Sources, F. Giovannelli (Ed.), SIDEREA, Roma, Italy, pp. 3-16.

[219] Smak, J.: 1985b, in Multifrequency Behaviour of Galactic Accreting Sources, F. Giovannelli (Ed.), SIDEREA, Roma, Italy, pp. 17-36.

[220] Soka Gakkai (Ed.): 1999, The writings of Nichiren Daishonin, Vol. I, p. 196.

[221] Spergel, D.N., Verde, L., Peiris, H.V., Komatsu, E., Nolta, M.R. et al.: 2003, ApJS, 148, Issue 1, 175-194.

[222] Stanev, T.: 2002, private communication.

[223] Steinhardt, P.J., Turok, N., Starkman, G.D.: 2008, PhT 61, Issue 1, 59.

[224] Straniero, O., Imbriani, G., Strieder, F., Bemmerer, D., Broggini, C. et al.: 2013, ApJ, 763, id. 100, $10 \mathrm{pp}$.

[225] Straniero, O., Bruno, C.G., Aliotta, M., Best, A., Boeltzig, A. et al.: 2017, A\&A 598, A128.

[226] Strieder, F., Limata, B., Formicola, A., Imbriani, G., Junker, M. et al.: 2012, PhL B, 707, Issue 1, 60-65.

[227] Tanvir, N.R., Fox, D.B., Levan, A.J., Berger, E., Wiersema, K. et al.: 2009, Nature, 461, 1254-1257.

[228] Tanvir, N.R., Levan, A.J., Fruchter, A.S., Hjorth, J., Hounsell, R.A. et al.: 2013, Nature, 500, 547-549. 
[229] Tonry, J.L., Schmidt, B.P., Barris, B., Candia, P., Challis, P. et al.: 2003, ApJ, 594, 1-24.

[230] Toshikawa, J., Kashikawa, N., Ota, K., Morokuma, T., Shibuya, T. et al.: 2012, ApJ, 750, Issue 2, article id. 137, 12 pp.

[231] Trezzi, D., Anders, M., Aliotta, M., Bellini, A., Bemmerer, D. et al. (LUNA Collaboration): 2017, Astrop. Phys. 89, 57-65.

[232] Turner, M.S., Riess, A.G.: 2002, ApJ 569, 18-22.

[233] Tyson, J.A.: 2000, Encyclopedia of Astronomy and Astrophysics, Edited by Paul Murdin, article 2144.

[234] Tyson, J.A., Kochanski, G.P., Dell'Antonio, I.P.: 1998, ApJL 498, Issue 2, L107-L110.

[235] United Nations, Dpt of Social and Economics Affairs, Population Division: 2004, World Population to 2300, ST/ESA/SER.A/236, New York, United Nations.

[236] United Nations, Dpt of Social and Economics Affairs, Population Division: 2017, in World Population Prospects: The 2017 Revision, New York, United Nations.

[237] de Vries, J., Archibald, J.M.: 2018, New Phytologist 217, 1428-1434.

[238] Wang, F.Y., Dai, Z.G.: 2009, MNRAS, 400, Issue 1, L10-L14.

[239] Wheatley, P.J., Mauche, C.W., Mattei, J.A.: 2003, MNRAS 345, 49.

[240] Wikipedia: 2017, online at https://en.wikipedia.org/wiki/Timeline underscore of underscore the underscore 2017 underscore Atlantic underscore hurricane underscore season

[241] Williams, L.L.R., Schechter, P.L.: 1997, arXiv:astro-ph/9709059, and Astron.\& Geophys. 38, Issue 5,10 .

[242] Wilmoth, J.R.: 1998, Science 280, Issue 5362, pp. 395-397.

[243] Wilmoth, J.R.: 2009, talk on 22 December at the Institute for Population and Social Security Research, Tokyo, Japan

[244] Winn, J.N., Rusin, D., Kochanek, C.S.: 2004, Nature 427, Issue 6975, 613-615.

[245] Winstein, B.: 2007, Int. J. Mod. Phy. D 16, Issue 12b, 2563.

[246] Winstein, B.: 2009, in From Quantum to Cosmos: Fundamental Physics Research in Space, Turyshev, Slava G. (Ed.), Published by World Scientific Publishing Co. Pte. Ltd., ISBN 9789814261210, pp. 697-705.

[247] Wittman, D.M., Tyson, J.A., Kirkman, D., Dell'Antonio, I., Bernstein, G.: 2000, Nature 405, Issue $6783,143-148$.

[248] Woese, C.R.: 1998, Proc. Natl. Acad. Sci. USA, Vol. 95, pp. 6854-6859.

[249] Woese, C.R.: 2000, Proc. Natl. Acad. Sci. USA, Vol. 97, no. 15, 8392-8396.

[250] Woese, C.R.: 2002, Proc. Natl. Acad. Sci. USA, Vol. 99, no. 13, 8742-8747.

[251] Wolschin, G.: 2003, in Time, Quantum and Information, L. Castell \& O. Ischebeck (Eds.), Springer, Berlin, Germany, p. 115.

[252] Zhang, B.: 2013a talk at the "Multi-Messenger Transient Workshop, KIAA, China.

[253] Zhang, B.: 2013b, in Gamma-ray Bursts: 15 Years of GRB Afterglows, A.J. Castro-Tirado, J.Gorosabel and I.H. Park (Eds.), EAS Publications Series 61, 285-293. 
[254] Zaroubi, S.: 2013, in The First Galaxies, ASSL, 396, 45-104.

\section{DISCUSSION}

VLADIMIR LIPUNOV's Comment: I would like to add to the bright opening talk only one important point. One of the most important discovery over the last few years is the Dark Energy detection in the local Universe by Arthur Chernin and Igor Karachentsev. This is the result of Hubble motion of the nearby galaxies at distance between 1 and $10 \mathrm{Mpc}$.

JAMES HOWARTH BEALL: Can you comment of the experimental (or observational) differences between an inflationary and an oscillatory universe?

That is, would there be any observational way to decide between these two possibilities?

FRANCO GIOVANNELLI: Classic inflation is for the first time also disfavored by data, specifically the recent data from WMAP, ACT and Planck2013.

Thus cyclic models of the universe have been discussed since they show the advantage of avoiding initial conditions problems related to postulating any sort of beginning in time and the advantage of not needing inflation (Ijjas, 2018). To date, the best known viable examples of cyclic models have been ekpyrotic (Erickson et al., 2007). One immediate prediction of cyclic theories is that the current dark energy dominating phase must be metastable or slowly decaying, ultimately transitioning to a state of lower energy density that will initiate a period of contraction. Cycling may also explain the magnitude of the dark energy density and other fundamental parameters (Ijjas $\&$ Steinhardt, 2018).

However, as far as we know there are no experimental tests for determining the history of dark energy density. On the contrary the inflationary model can be proved if B-mode polarization would be unequivocally confirmed. 